Draft version April 2, 2021

Typeset using $\mathrm{LAT}_{\mathrm{E}} \mathrm{X}$ twocolumn style in AASTeX63

\title{
EXOPLINES: Molecular Absorption Cross-Section Database for Brown Dwarf and Giant Exoplanet Atmospheres
}

\author{
Ehsan Gharib-Nezhad,${ }^{1,2,3, *}$ Aishwarya R. Iyer,${ }^{4}$ Michael R. Line, ${ }^{4}$ Richard S. Freedman, ${ }^{5,1}$ Mark S. Marley, \\ and Natasha E. Batalha ${ }^{1}$ \\ ${ }^{1}$ NASA Ames Research Center, Moffett Field, CA 94035, USA \\ ${ }^{2}$ Bay Area Environmental Research Institute, NASA Ames Research Center, Moffett Field, CA 94035, USA \\ ${ }^{3}$ School of Molecular Sciences, Arizona State University, Tempe, AZ 85281, USA \\ ${ }^{4}$ School of Earth and Space Exploration Arizona State University, Tempe AZ 85281, USA \\ ${ }^{5}$ SETI Institute, Mountain View, CA 94035, USA
}

\begin{abstract}
Stellar, substellar, and planetary atmosphere models are all highly sensitive to the input opacities. Generational differences between various state-of-the-art stellar/planetary models are primarily because of incomplete and outdated atomic/molecular line-lists. Here we present a database of precomputed absorption cross-sections for all isotopologues of key atmospheric molecules relevant to latetype stellar, brown dwarf, and planetary atmospheres: $\mathrm{MgH}, \mathrm{AlH}, \mathrm{CaH}, \mathrm{TiH}, \mathrm{CrH}, \mathrm{FeH}, \mathrm{SiO}$, $\mathrm{TiO}$, $\mathrm{VO}$, and $\mathrm{H}_{2} \mathrm{O}$. The pressure and temperature ranges of the computed opacities are between $10^{-6}$ 3000 bar and 75-4000 K, and their spectral ranges are $0.25-330 \mu \mathrm{m}$ for many cases where possible. For cases with no pressure-broadening data, we use collision theory to bridge the gap. We also probe the effect of absorption cross-sections calculated from different line lists in the context of Ultra-Hot Jupiter and M-dwarf atmospheres. Using 1-D self-consistent radiative-convective thermochemical equilibrium models, we report significant variations in the theoretical spectra and thermal profiles of substellar atmospheres. With a $2000 \mathrm{~K}$ representative Ultra-Hot Jupiter, we report variations of up to 320 and $80 \mathrm{ppm}$ in transmission and thermal emission spectra, respectively. For a $3000 \mathrm{~K} \mathrm{M}$-dwarf, we find differences of up to $125 \%$ in the spectra. We find that the most significant differences arise due to the choice of $\mathrm{TiO}$ line-lists, primarily below $1 \mu \mathrm{m}$. In sum, we present (1) a database of pre-computed molecular absorption cross-sections, and (2) quantify biases that arise when characterizing substellar/exoplanet atmospheres due to line list differences, therefore highlighting the importance of correct and complete opacities for eventual applications to high precision spectroscopy and photometry.
\end{abstract}

Keywords: Brown dwarfs (185), Exoplanet atmospheres (487), Exoplanet atmospheric composition (2021)

\section{INTRODUCTION}

Over the past two decades, atmospheric characterization of exoplanets and brown dwarfs has been dramatically increasing - particularly, observations with $H S T$, Spitzer, various ground-based telescopes both with low to moderate resolution spectroscopy and high-resolution cross-correlation approaches (e.g., Madhusudhan 2019). Additionally, with the Transiting Exoplanet Survey Satellite (TESS), in conjunction with the launch of

Corresponding author: Ehsan Gharib-Nezhad

e.gharibnezhad@asu.edu

* NASA Postdoctoral Fellow the James Webb Space Telescope (JWST), we can expect hundreds of planet detections (Sullivan et al. 2015; Louie et al. 2018; Kempton et al. 2018) followed by detailed spectroscopic characterization of their atmospheres (Greene et al. 2016; Bean et al. 2018). The compositional and temperature diversity of these worlds provide a unique opportunity to gain insight into planetary atmospheric chemical processes and formation pathways (Öberg et al. 2011; Fortney et al. 2013; Mordasini et al. 2016; Hörst et al. 2018).

Fully capitalizing on the information encoded in a spectrum, therefore, requires a robust model interpretation. Models used to interpret thermal emission spectra (either from an isolated object like a brown dwarf, M- 
dwarf, or directly imaged planet, or a secondary eclipse spectrum for a transiting planet) and transmission spectra (for transiting planets) require some combination of chemistry and radiative transfer. Self-consistent models (either $1 \mathrm{D}$ or $3 \mathrm{D}$ ) rely upon a detailed coupling between chemical processes (e.g., thermochemical equilibrium or chemical kinetics/photochemistry), radiative energy transport, convective/dynamical energy transport, and cloud/haze processes to determine the atmospheric state and subsequent observables (e.g., Marley \& Robinson 2015; Zhang 2020, and references therein). Retrieval models, on the other hand, are purely data-driven and attempt to leverage large numbers of free parameters (e.g., molecular abundances for selected species, temperature profile parameterization) to adequately fit the observables to determine Bayesian constraints on composition, clouds, and thermal structures (Line et al. 2014). The adequacy of these methods, whether for predictions or parameter estimation, relies upon accurate and complete molecular and atomic opacities.

In this work, we focus on high-resolution (adaptive R, e.g., $2 \times 10^{4}-2 \times 10^{6}$ at $1 \mu \mathrm{m}$ ) opacities with implications to Ultra-Hot Jupiter atmospheres. Our prime candidates being $\mathrm{TiO}, \mathrm{VO}, \mathrm{MgH}, \mathrm{AlH}, \mathrm{CaH}, \mathrm{TiH}, \mathrm{CrH}$, $\mathrm{FeH}$, and $\mathrm{SiO}-$ the key molecules ${ }^{1}$ in such atmospheres. In addition, we generate $\mathrm{H}_{2} \mathrm{O}$ high-resolution opacities from 500-4000 K, which is required for modeling everything from super-Earth to M-dwarf atmospheres. Starting with $\S 2$, we first provide an astrophysical context for the importance of metal hydrides and oxides in substellar atmospheres. $\S 3$ provides discussion on the current state of opacity or absorption cross-section data, highlighting line broadening effects that are missing and how we tackle them. In $\S 4$, we bridge the gap by providing pre-generated absorption cross-sections for the metal hydrides and oxides in question, alongside comparisons with previous efforts. In $\S 5$, we quantify the effect of opacity differences on transmission and emission spectra of a representative ${ }^{2}$ Ultra Hot Jupiter planet, followed by M-dwarf stellar spectra in $\S 6$, and potential biases and challenges with opacities that arise in atmospheric characterization efforts, described in $\S 7$. Finally, we present a brief summary of our conclusions in $\S 8$. The main outcome of this work is EXOPLINES absorption cross-section database (i.e., EXOplanet Pressurebroadened LINES).

\footnotetext{
${ }^{1}$ For further study on the composition of these ultra-hot Jupiters and M-dwarfs, studies by Lodders \& Fegley (2006) and Visscher et al. (2010) are suggested.

2 "representative" in this context means a generic atmospheric case
}

\section{ASTROPHYSICAL IMPORTANCE}

Temperature and pressure play a key role in controlling molecular and atomic abundances that ultimately shape a spectrum (e.g., Burrows \& Sharp 1999). At cooler temperatures $(<200 \mathrm{~K})$, solar system giants are dominated by absorption because of methane and scattering by clouds, and photochemically produced hydrocarbons. At warmer temperatures (300-1000 K), Tdwarfs, and many (sub)Neptune transiting planet atmospheres are sculpted (or presumed to be sculpted) by water, methane, ammonia, and alkali metals. Higher temperature results in the onset of $\mathrm{CO}$ absorption, followed by several oxides ( $\mathrm{TiO}, \mathrm{VO})$ and hydrides $(\mathrm{FeH}$, $\mathrm{CrH}, \mathrm{MgH}, \mathrm{CaH})$, especially above $1500 \mathrm{~K}$. The increasing presence of these species as a function of temperature is what ultimately (along with dust/condensate formation) governs the M-L-T-Y dwarf classification sequence and their sub-types (Kirkpatrick 2005). Furthermore, water dissociation, the onset of H-bound/freefree, and contribution of refractory atoms and their ions (e.g., Fe, Ti, Mg, Ca, Si) dominate atmospheres with temperatures above $\sim 2500 \mathrm{~K}$, encountered in Mdwarfs and ultra-hot Jupiters (Lodders 1999). Here, we highlight the importance of these metal oxides and hydrides and their behavior in high-temperature regimes - particularly in substellar atmospheres of M/Ltype Dwarfs and highly irradiated exoplanets.

\subsection{Metal Oxides}

M-dwarf stars $\left(2000 \mathrm{~K}<\mathrm{T}_{\text {eff }}<4000 \mathrm{~K}\right)$, active photospheric spots on sun-like stars, and even highly irradiated exoplanets with similar effective temperatures have atmospheres that are all dominated by TiO (Kirkpatrick et al. 1991; Webber 1971). Covering the optical spectral region between $630-900 \mathrm{~nm}$, TiO plays a vital role in the proper characterization of M-dwarfs and UltraHot Jupiters. The temperature dependence of TiO governs the energy level populations that affect the relative band-structure intensities, an essential tool in classifying early-type M-dwarfs (Sharpless 1956). For instance, in spectra of M0-M3 dwarfs, the 0-0 band ${ }^{3}(705.4 \mathrm{~nm})$ is used as the primary classification marker. The strongest TiO bands near $843 \mathrm{~nm}, 819.4 \mathrm{~nm}$ to $885.9 \mathrm{~nm}$, and 660 to $710 \mathrm{~nm}$ are important indicators for M4, M6, and late-type M8 dwarfs respectively (Sharpless 1956; Lockwood 1972; Ruiz et al. 1997; Delfosse et al. 1997).

Similar to $\mathrm{TiO}, \mathrm{VO}$ is a refractory molecule in Mdwarfs and early L-dwarf atmospheres ( L2). With

\footnotetext{
${ }^{3}$ In spectroscopy, the $\nu^{\prime}-\nu^{\prime \prime}$ band refers to all rotation-vibrational transitions between the upper vibrational level, $\nu^{\prime}$, and the lower vibration level $\nu^{\prime \prime}$ (Bernath 2005).
} 
strong spectral signatures between 0.6-1.5 $\mu \mathrm{m}$, metal oxides such as VO are sensitive temperature indicators in substellar atmospheres (Cushing et al. 2005). The strength of the VO absorption features at 1.17 and $1.22 \mu \mathrm{m}$, combined with $\mathrm{TiO}$ and $\mathrm{CaH}$ bands, are valuable spectroscopic youth indicators for brown dwarfs (Kirkpatrick et al. 2006; Peterson et al. 2008).

In addition to their importance for substellar spectral and age classification, high-temperature oxides are critical sources of opacity in the optical wavelength region, that drive heating in the upper atmospheres of hot Jupiters ( $>1800 \mathrm{~K}$ and $<50$ mbar) - essential for proper characterization of the $1 \mathrm{D}$ vertical structure of the atmosphere (Hubeny et al. 2003; Fortney et al. 2008). The presence of $\mathrm{TiO}$ and $\mathrm{VO}$ drive up stellar irradiation mean opacity relative to the planetary thermal emission mean opacity, resulting in a "thermal inversion" (increasing temperature with decreasing pressure) in the planetary atmosphere. However, we note that bonafide detection of metal oxides ( $\mathrm{TiO}$ or $\mathrm{VO}$ ) has remained elusive and somewhat controversial, partially due to the inadequacy of the opacity data for these species (Merritt et al. 2020; Nugroho et al. 2017).

In substellar atmospheres, especially at temperatures cooler than $2300 \mathrm{~K}$, both $\mathrm{TiO}$ and VO gradually disappear due to condensate formation ( $\mathrm{TiO}$ into to $\mathrm{CaTiO}_{3}$ and VO turns into solid VO, Lodders (1999)). Therefore, their spectral features are not prominent at such atmospheres at $\sim 0.7-1.2 \mu \mathrm{m}$, allowing for metal hydride $\mathrm{FeH}, \mathrm{CrH}$, and $\mathrm{CaH}$ features to present themselves.

\subsection{Metal Hydrides}

Several FeH features within the $0.7-1.3 \mu \mathrm{m}$ range has been detected in the late-M and $\mathrm{L}$ dwarfs (Kirkpatrick et al. 2000), including the 0-0 band (known as the WingFord band) at 0.985-1.02 $\mu \mathrm{m}$ (Cushing et al. 2003), the 1-0 band at $869.2 \mathrm{~nm}$ in L-dwarfs (Kirkpatrick et al. $1999)$, the $2-0$ band at $778.6 \mathrm{~nm}$, the $1-0$ band at $869.2 \mathrm{~nm}$, the $2-1$ band at $902.0 \mathrm{~nm}$ (Tinney \& Reid 1998) and the 1-2 band at $1238.9 \mathrm{~nm}$ bands are present in very low-mass stars (Reid et al. 2001). Additionally, Schiavon et al. (1997) showed that the Wing-Ford band is sensitive to surface gravity and metallicity. Similar to $\mathrm{FeH}, \mathrm{CaH}, \mathrm{CrH}$, and $\mathrm{AlH}$ all show a strong dependence on surface gravity (see Chapter 4 in Reid \& Hawley (2005)).

The CrH 0-0 (861.1 nm) and 0-1 (996.9 nm) bands along with the $\mathrm{FeH}$ bands (and their ratios) serve as diagnostic indicators (Kirkpatrick et al. 1999, 2000) for classifying the L-dwarf sub-types (see Table 6 in Kirkpatrick et al. (1999)).
In the context of Ultra-Hot Jupiters, species like MgH, $\mathrm{AlO}, \mathrm{NaH}$, and $\mathrm{CaO}$ could also drive thermal inversions depending on elemental abundance ratios (Gandhi \& Madhusudhan 2019). Additionally, hydrides such as $\mathrm{MgH}, \mathrm{CrH}, \mathrm{CaH}$, and $\mathrm{FeH}$ although expected to be present in hot Jupiter atmospheres; the lack of reliable detections have made it difficult to assess their true role in governing the 1-D thermal structure.

\section{METHODOLOGY: MOLECULAR ABSORPTION CROSS-SECTIONS}

The absorption cross section ${ }^{4}$ (hereafter ACS) of an absorber is governed by the wavelength-dependent intensity, position, and width of a spectral line. The line shape is typically modeled with a Voigt profile, which is a convolution of Doppler (thermal) and pressure (Lorentz or collisional) broadening. Uncertainties in the line positions, strengths, and widths are ultimately what drive the uncertainties or inadequacies in various precomputed ACSs. Below we present the formulation of line profile in $\S 3.1$, and then we extend our discussion on pressure-broadening and their availability in $\S 3.2$, followed by the treatment for their $J$-dependency and wing cut-off in §3.3-3.4. Finally, §3.5 describes the line-list data structure, and computational details are provided in $§ 3.6$.

\subsection{Line-width}

The conventional form of the spectral line shape is the Voigt profile, which is a convolution of Doppler and Lorentz profiles. Doppler broadening is governed by the temperature, molecular mass, and also the transition energy, and its half-width at half-maximum width (HWHM), $\Gamma_{\mathrm{D}}$, may be given by (Buldyreva et al. 2011):

$$
\Gamma_{\mathrm{D}}=3.581 \times 10^{-7} \sqrt{\frac{T}{M}} \nu
$$

where $M$ is absorbers molecular mass in amu (atomic mass unit), $T$ is the temperature (in $\mathrm{K}$ ), and $\nu$ is the line position in wavenumber $\left(\mathrm{cm}^{-1}\right)$.

Lorentz line profile is controlled by the magnitude of the interaction between the absorber and broadening gas species, and the Lorentz HWHM, $\Gamma_{\mathrm{L}}$, depends on

\footnotetext{
${ }^{4}$ Both opacities and absorption cross-sections represent the capability of species (i.e., atom, molecule, or radical) to absorb or emit light. However, their definitions are different. Opacity $\kappa$ is the "effective area" of one gram of an absorber (unit: $\mathrm{cm}^{2} / \mathrm{gram}$ ) to absorb/emit light while absorption cross-section $\sigma$ is the "effective area" of one absorber (unit: $\mathrm{cm}^{2} /$ molecule). These quantities are interchangeable with $\kappa \rho=\sigma n$ equation, where $\rho$ is mass density $\left(\mathrm{gram} / \mathrm{cm}^{3}\right)$ and $n$ is number density (molecule $/ \mathrm{cm}^{3}$ ).
} 
the temperature and the broadening species and can be described by

$$
\Gamma_{\mathrm{L}}=\sum_{b}\left(\frac{T}{296 \mathrm{~K}}\right)^{-n_{T, b}} \gamma_{\mathrm{L}, b} p_{\mathrm{b}}
$$

where $\gamma_{\mathrm{L}, \mathrm{b}}$ is the Lorentz coefficient of an absorber (HWHM, unit: $\mathrm{cm}^{-1} / \mathrm{bar}$ ), $p_{\mathrm{b}}$ is the broadener partial pressure (unit: bar), and $n_{\mathrm{T}, b}$ is the temperaturedependence coefficient, which is dimensionless. Both $\gamma_{\mathrm{L}, \mathrm{b}}$ and $n_{\mathrm{T}, b}$ are controlled by broadener-absorber interaction and the quantum properties of absorbing molecules.

Quantifying $\gamma_{\mathrm{L}, b}$ and $n_{\mathrm{T}, b}$ coefficients for the metal hydrides/oxides in question (e.g., $\mathrm{TiO}, \mathrm{VO}, \mathrm{MgH}$, etc.) are challenging aspects of ACS calculations because there is not any laboratory or computational measurements have been done to determine their $\gamma_{\mathrm{L}, b}$ and $n_{\mathrm{T}, b}$ coefficients $^{5}$.

\subsection{Estimating the Lorentz Coefficient}

Theoretically determining the Lorentz coefficient is quite challenging because it depends on molecular quantum properties such as total rotational angular momentum $^{6}$, molecular symmetries, and environmental conditions like temperature and the background gas broadener composition. For TiO, VO, TiH, CrH, the Lorentz coefficients have been estimated up to low $J$ quantum numbers (given in Table 1 ) for $\mathrm{H}_{2} / \mathrm{He}$ atmospheres. These estimations are based on the similarity between the molecular symmetry or dipole moments that these absorbers have with the available Lorentz coefficients (e.g., Freedman et al. 2014; Chubb et al. 2020) ${ }^{7}$ ). In reality, we do not have enough or even a single measurement for the metal hydries/oxides in this study (except $\mathrm{H}_{2} \mathrm{O}$ ) at any temperature (see $\S 7$ for the challenges we are dealing with these opacities).

Due to the lack of $\gamma_{\mathrm{L}, b}$ and $n_{\mathrm{T}, b}$ coefficients, we used the classical collision theory by Anderson (1949) in order

${ }^{5}$ More precisely, we could find only one spectroscopic measurement of AlH in $\mathrm{H}_{2}$ gas by Watson \& Hull (1936). Their measured $\gamma_{\mathrm{L}}$ is $0.24 \mathrm{~cm}^{-1} / \mathrm{atm}$, which looks much larger than the available $\mathrm{H}_{2}$-broadening data for other well-known absorbers. Given the limitation in pressure gauges, spectrometers, and thermometers in their measurements in the 1930s, we decided not to use their number.

${ }^{6}$ In fact, each transition has its corresponding rotational and vibrational quantum numbers and molecular symmetries, which are controlling the Lorentz coefficient and hence the spectral linewidth.

${ }^{7}$ For instance, Chubb et al. (2020) used HCN broadening coefficients for computing the ACS data of $\mathrm{CaH}, \mathrm{CrH}, \mathrm{SiO}, \mathrm{TiO}$, and VO absorbers, as well as HF broadening coefficients data for $\mathrm{FeH}$ and TiH. to estimate the Lorentz coefficient using the following equation (see $\S$ A.1 for further discussion):

$$
\gamma_{\mathrm{L}, \mathrm{b}}=0.0567(T \mu)^{-1 / 2} \sigma_{\mathrm{col}, \mathrm{b}}
$$

where $\mu$ is the reduced mass of the absorber-broadener pair in amu, $\sigma_{\text {col,b }}$ is the collision cross-section in $\AA^{2}$ of the broadener $\mathrm{b}$, and $\gamma_{\mathrm{L}, \mathrm{b}}$ is in $\mathrm{cm}^{-1} / \mathrm{atm}$. In this equation, the collision (or scattering) cross section, $\sigma_{\text {col,b }}$ is a fundamental factor, that connects collisions in the microscopic world into the spectral linewidth. For further details, see studies by Odashima et al. (1989) and Gierszal et al. (1998) that have used this approach to calculate the collision cross section from the laboratorymeasured Lorentz line width. In addition, Cappelletti et al. (2005) have shown a good agreement between the experimental and computed pressure-broadening coefficients of $\mathrm{C}_{2} \mathrm{H}_{2}-\mathrm{Ar}$ system.

Even though Eq. 3 is derived from the collision theory, it has some limitations and errors such as ideal gas assumption and also possible inaccuracy in the computed collision cross-section data from ab-initio methods. Hence, this equation and the calculated pressurebroadening coefficients will not fulfill the lack of laboratory measurements or theoretical calculations. For example, the modified semi-classical Robert Bonamy theory (Robert \& Bonamy 1979; Ma et al. 2007) have been successfully implemented to calculate the half-width and line shift of $\mathrm{H}_{2} \mathrm{O}$ and $\mathrm{CO}$ absorbers in different broadening gases (e.g., Gamache \& Vispoel 2018; Vispoel et al. 2020), and so similar studies are required for these molecules in question.

Note that the assumption of 0.5 for the temperaturedependence coefficient, $n_{\mathrm{T}, \mathrm{b}}$ at Eq. 3 is based on the kinetic theory, while laboratory measurements of other species suggest $n_{\mathrm{T}, \mathrm{b}}$ can vary from $0.2-1.2$. In turn, $n_{\mathrm{T}, \mathrm{b}}$ can strongly depend on the broadener and $J$ quantum number (e.g., $\mathrm{CH}_{4}$ in different broadening gas that have shown in table 5 by Gharib-Nezhad et al. (2019)), or $n_{\mathrm{T}, \mathrm{b}}$ may be roughly constant with $J$ (e.g., CO-self/air broadening by Devi et al. (2012)). Wagner et al. (2005) have measured the effect of $\mathrm{H}_{2} \mathrm{O}$-air mixture and found that $n_{\mathrm{T}, \mathrm{b}}$ can also be negative number. Table 2 represents the collected collision cross sections, $\sigma_{c o l}$ for $\mathrm{CaH}$ and $\mathrm{SiO}$ absorbers for $\mathrm{H}_{2}$ or He broadeners where available for $J_{\text {lower }}=0$. In addition, the $\mathrm{AlH} \gamma_{\mathrm{L}, \mathrm{b}}$ coefficient is assumed to be equal to the CO broadening coefficient because of their similar dipole moments (we adopt this approach from Freedman et al. (2014) and Chubb et al. (2020) approaches.) 


\subsection{Estimating the Behavior of Pressure-Broadening Coefficients and Their Dependence on J Quantum Numbers}

The calculated or estimated Lorentz coefficients, $\gamma_{\mathrm{L}}(J)$ depend on $J$ quantum numbers. To understand the behavior of $\gamma_{\mathrm{L}}(J)$ for all $J_{\mathrm{s}}$, we need to consider two issues: 1) the degree or rate of the $\gamma_{\mathrm{L}}(J)$ decrease with $J$ (or $\frac{\partial \gamma}{\partial J}$ ), and 2) an equation that can extrapolate these $J$-dependence behavior without becoming negative or increasing sharply.

The first issue can be tackled by comparing the $J$ dependence behavior of the available $\gamma_{\mathrm{L}}(J)$ coefficients of diatomic molecules with $\mathrm{H}_{2}$ and He broadening. For example, the Lorentz coefficient of $\mathrm{CO}$ molecule (with low dipole moment) has a smooth decrease with high $J_{\mathrm{s}}$ (Devi et al. 2004; Régalia-Jarlot et al. 2005; Mantz et al. 2005). In contrast, for absorbers with large dipole moments such as $\mathrm{HCl}-\mathrm{H}_{2}$ and $\mathrm{HCl}-\mathrm{He}$ systems, $\gamma_{\mathrm{L}}(J)$ value drop noticeably according to the laboratory measurements done by Toth et al. (1970), Li et al. (2018), Babrov et al. (1960) (see Fig. 1). All the metal hydrides and oxides in question (except $\mathrm{AlH}$ ) have large dipole moments, and for the lack of any other information we assume that their $J$-dependency may decrease similar to $\mathrm{HCl}$. However, one should keep in mind that dipole moment values are not major factors in the rotational dependence of the broadening parameters. Detailed discussion on the reason for $J$-dependence of spectral lines is provided by Hartmann et al. (2021) (chapter 3) and Renaud et al. (2018). The second issue may be solved by using the proposed equation by the HITRAN group ${ }^{8}$ (e.g., see Gordon et al. 2017, and their upcoming paper, Gordon et. al. 2021). As part of their group efforts, Tan et al. (2019) have examined the fitting accuracy of the available pressure-broadening coefficients for water as a broadener and several terrestrial key absorbers (e.g., $\mathrm{CO}, \mathrm{CO}_{2}, \mathrm{CH}_{4}$ ); and they suggested the fourthorder Padé approximant as the most reliable prediction of the Lorentz $J$-dependency. Fourth-order Padé equation provides a smooth decrease of $\gamma_{\mathrm{L}}$ with $J$ and has the following form:

$$
\gamma_{\mathrm{L}}(J)=\frac{a_{0}+a_{1} J+a_{2} J^{2}+a_{3} J^{3}}{1+b_{1} J+b_{2} J^{2}+b_{3} J^{3}+b_{4} J^{4}}
$$

where $a_{0}$ to $a_{3}$ and $b_{1}$ to $b_{4}$ are the fitting coefficients. $J$ is also the lower total angular quantum number. Besides, we applied the following constraints to this equation to avoid negative numbers of $\gamma_{\mathrm{L}}$ as well as follow

8 https://www.hitran.org a sharp $J$-dependency for absorbers with large dipole moments (i.e., $\mathrm{MgH}, \mathrm{CaH}, \mathrm{CrH}, \mathrm{FeH}, \mathrm{TiH}$ ):

$$
\begin{aligned}
& \gamma_{\mathrm{L}}(J=10)=\sim 0.5 \gamma_{\mathrm{L}}(J=0) \\
& \gamma_{\mathrm{L}}(J=40)=\sim 0.1 \gamma_{\mathrm{L}}(J=0) \\
& \text { if } \gamma_{\mathrm{L}}(J>40)<0=>\gamma_{\mathrm{L}}(J)=\gamma_{\mathrm{L}}^{\text {min }}-\left(\frac{1}{J} * \gamma_{\mathrm{L}}^{\text {min }}\right)
\end{aligned}
$$

An example of the calculated broadening coefficients for the metal hydrides/oxides in question is presented in Fig. 2. Additionally,, the Fourth-order Padè coefficients for all the absorbers in this study are listed in Table 3 and are valid up to $J=500$.

\subsection{Wing cut-off}

Wing cut-off is an important challenge in fully and accurately computing the opacity continuum (see $\S 7$ ). Given the lack of sufficient pressure broadening data for these species and the huge computational time, the Voigt profile is implemented in various pre-computed opacity data. The current method to deal with this nonLorentzian behavior of the Voigt profile is either using absolute wavenumber such as $100 \mathrm{~cm}^{-1}$ (e.g., MacDonald 2019) or just using multiplication of the Voigt profile (e.g., 200 to 500 of Voigt HWHM) (e.g., Chubb et al. 2020; Freedman et al. 2014).

Note that several laboratory and theoretical studies have shown that non-Voigt behavior provides a more accurate opacity continuum (e.g., Ngo et al. 2012). For example, Hartmann et al. (2002) have recorded the $3.3 \mu \mathrm{m}$ spectrum of $\mathrm{CH}_{4}$ in $\mathrm{H}_{2}$ bath gas and have shown that the Voigt behavior is valid up to $25 \mathrm{~cm}^{-1}$ from the line center. Inspiring by their results, we decide to use absolute number for the wing cut-off as follow: $30 \mathrm{~cm}^{-1}$ for $P \leq 200$ bar and $150 \mathrm{~cm}^{-1}$ for $P>200$ bar. We noticed that Mollière et al. (2019) have considered this method as well in calculating their opacities.

It is worth mentioning that we did not use the multiplication factor of the Voigt linewidth because for a generic spectral line at low-to-moderate pressure and at the infrared spectral region with small wavenumber (such as $P<10^{-3}$ bar at $<1000 \mathrm{~cm}^{-1}$ ), the $500 \times$ Voigthalf-width will be less than $10 \mathrm{~cm}^{-1}$ which is not sufficiently high value for the wing cut-off according to the results by Hartmann et al. (2002). Additionally, for high pressures (such as $P>100$ bar), this $500 \times$ Voigt-HWHM is a large number even larger than $500 \mathrm{~cm}^{-1}$ which results in an extreme overestimation.

\subsection{Line List}

A typical line-list data contains all of the necessary ingredients to generate ACSs, such as line positions, 
intensities, rotational and vibrational quantum assignments, electronic assignments, and also various pressurebroadening and pressure-shift values where available. The completeness and accuracy of line-lists are a key focus of both laboratory and $a b$ initio studies. In this work, we use the latest version of line list data provided by the ExoMol group (Tennyson et al. 2020), summarized in Table 4.

\subsection{Computational Details}

We employ the ExoCross ${ }^{9}$ code developed by Yurchenko et al. (2018) to compute the temperaturedependent line strengths and the Voigt profile (Humlíček 1979) for every individual line under the conditions given in Table 5. We assume $\mathrm{H} / \mathrm{He}$ broadening governed by background atmospheres composed of $85 \% \mathrm{H}_{2}$ and $15 \% \mathrm{He}$-typical Jovian-like atmosphere conditions. The spectral sampling resolution is optimized as a function of temperature, pressure, and spectral subdivisions in such a way as to fully resolve the individual lines.

\section{RESULTS: ABSORPTION CROSS-SECTION}

In this section, we present a brief discussion on the line-list source for each absorber, as well as illustrative examples of the pre-computed ACS data. Then, we compare our generated ACS data with the currently available database's.

\section{1. $\mathrm{H}_{2} \mathrm{O}$}

Partridge \& Schwenke (1997) used high-level ab initio approach to computing the potential energy surface and dipole moment function of water. They then empirically adjusted their water line list (hereafter, $P S 97$ ) by comparing their line position with available laboratory spectra. About a decade later, the ExoMol team (Barber et al. 2006) computed the BT2 water line list, which includes $\sim$ twice as many transitions with a somewhat lower total angular quantum number $J$ but over a wider spectral range, extending deeper into the blueoptical. However, the comparison of both line lists with laboratory $\mathrm{H}_{2} \mathrm{O}$ measurements showed that the PS97 ACSs are more accurate in predicting line positions than BT2 at wavelengths longer than $1 \mu \mathrm{m}$ (Alberti et al. 2015; Melin \& Sanders 2016). It is for this reason that the $P S 97$ list has been used in several works (e.g., Freedman et al. (2014); Marley \& Robinson (2015)), despite more modern line lists. Meanwhile, HITEMP - the high-temperature arm of the CfA HITRAN database - was created, using a trimmed version

\footnotetext{
${ }^{9}$ github.com/Trovemaster/exocross/wiki
}

of the $B T 2$ line list, but with rigorous validation and adjustments against laboratory data (Rothman et al. 2010). Recently, ExoMol improved the BT2 line list by refining the potential energy surfaces, and extending the wavelength range down to $0.25 \mu \mathrm{m} \quad(40,000$ $\mathrm{cm}^{-1}$ ), referred to as the POKAZATEL (Polyansky et al. 2018) line list. Table 6 summarizes these line lists and their key differences, and Fig. 3 compares the resultant cross-sections for a representative pressure/temperature. Besides the spectral range, there are extensive differences in their line positions, line shifts with pressure, and the number of lines, clearly apparent in different spectral regions such as the $2.420-2.424 \mu \mathrm{m}$ band ( $\mathrm{K}$ band)-subject to numerous high-resolution observations (e.g., Birkby et al. (2013)). Brogi \& Line (2019) showed that these differences matter in constructing high-resolution spectroscopy cross-correlation functions potentially leading to bias in the interpretation of the atmospheric composition. Recently, Gandhi et al. (2020) compared the water POKAZATEL data with the laboratory HITEMP data, also in the context of high-resolution cross-correlation spectroscopy, showing very good agreement up to temperatures of $\sim 1200 \mathrm{~K}$.

In our ACS database, we used the POKAZATEL $\mathrm{H}_{2} \mathrm{O}$ line list over the full spectral range of $0.25-100 \mu \mathrm{m}$ at the temperature and pressure conditions given in $\mathrm{Ta}$ ble 5. Figure 4 shows a subset of our ACSs as a function of temperature.

More recently, Conway et al. (2020) have published their $\mathrm{H}_{2} \mathrm{O}$ line list calculated from experimental spectroscopic data, which includes $\sim 10^{6}$ transitions and it is limited up to $J_{\max }=20$. Their line lists have shown a significant improvement in the line position for the ultraviolet spectral region. Note that the current ground-based telescopes with very high resolution are mostly detecting in the near-IR region; however, future telescopes in the UV-Vis regions are required to have accurate line positions for high-resolution cross-correlation modeling of observational data.

\section{2. $\mathrm{TiO}$}

Several spectroscopic measurements have been carried out to determine the spectroscopic parameters such as electronic dipole moments, hyperfine spectral properties, and rotational and vibrational constants for the 
$\mathrm{TiO}$ electronic ground state ${ }^{10}, X^{3} \Delta$, as well as the low-lying excited states such as $B^{3} \Pi, C^{3} \Delta$, and $D^{3} \Pi$ (Steimle \& Virgo 2003; Amiot et al. 1995; Balfour et al. 1993). In addition, several laboratory measurements have been done to determine the line position and strength between the following $\mathrm{TiO}$ electronic states: $\left(C^{3} \Delta-X^{3} \Delta\right)$ (Hodges \& Bernath 2018), $\left(c^{1} \Phi-a^{1} \Delta\right)$ (Bittner \& Bernath 2018), ( $\left.b^{1} \Pi-a^{1} \Delta\right)$ (Ram et al. 1996; Bittner \& Bernath 2018), $\left(A^{3} \Pi-X^{3} \Delta\right)$ (Ram et al. 1999), $\left(f^{1} \Delta-a^{1} \Delta\right)$ (Brandes \& Galehouse 1985), and $\left(c^{1} \Phi-a^{1} \Delta\right)$ (Bittner \& Bernath 2018).

Kurucz (1992) collected the available laboratory spectroscopic data for all $\mathrm{TiO}$ transitions to make the first comprehensive laboratory $\mathrm{TiO}$ line list (hereafter TiOKurucz92). Concurrently, Plez et al. (1992) used a theoretical spectroscopic method to calculate the transition dipole moments to generate $\mathrm{TiO}$ opacities. A few years later, they used more accurate dipole moments from Langhoff (1997) as well as the available spectroscopic measured data to improve line positions and intensities of the TiO nine low-lying electronic states (Plez 1998).

Schwenke (1998) independently used ab initio methods that included the spin-orbit and rotation-orbit coupling to compute potential energy surfaces for 13 electronic states. These surfaces were used as a Rydberg-Klein-Rees (RKR) potential to extract rovibrational energy levels. Schwenke (1998) also adopted the transition moments from Langhoff (1997) to determine the intensities for each rovibrational transition in order to compute a complete line list (intensities and positions) for $45 \times 10^{6}$ lines. Allard et al. (2000) compared these two (Plez92 and Schwenke98) TiO line lists in the context of M- and Brown-dwarf atmospheres, finding that the Schwenke98 data could better model the thermal emission from cool substellar atmospheres.

Hoeijmakers et al. (2015) used Schwenke98 and Plez92 line lists to detect/identify $\mathrm{TiO}$ in the HD 209458b atmosphere via high-resolution crosscorrelation spectroscopy $(\mathrm{R} \approx 100,000)$ finding that the line positions in both lists are not sufficient to extract an accurate signal. These mounting inadequacies in line positions and intensities motivated the need for a better,

\footnotetext{
${ }^{10}$ In diatomic spectroscopy, in general, the electronic ground state is represented by $X$ and the higher electronic states are labeled by $A, B$, and so on. In addition, the electronic multiplicity $(2 \mathrm{~S}+1)$ and the total orbital angular momentum $(\Lambda)$ are used to represent the electronic state. For example, $X^{3} \Delta$ is the ground state of $\mathrm{TiO}$ with a multiplicity of 3 and orbital angular momentum of $\Delta$, which is equivalent to $\Lambda=2$. Hence, $C^{3} \Delta-X^{3} \Delta$ is showing the electronic transition between the $\mathrm{TiO}$ state $C$ and $X$, with the same total orbital and spin angular momentums. Chapter 9 by Bernath (2005) provided detailed information on this topic.
}

more comprehensive, TiO line list. ExoMol used a highquantum level of basis set computation methods with spin-orbit coupling to generate all rovibronic (rotationvibration-electronic) transitions between the 13 lowlying electronic states, covering a spectral range of 0.33 $100 \mu \mathrm{m}$ (called TOTO, McKemmish et al. (2019a)). In our ACS database we used this TOTO line list ${ }^{11}$ from 0.3-100 $\mu \mathrm{m}$ over the conditions given in Table 5. Figure 5 summarizes a subset of these ACSs and Figure 6 and 7 compare to past line lists using identical computational setups.

More recently, Bernath (2020a) have recorded the TiO spectra in the visible-near-infrared regions (476-1176 $\mathrm{nm})$ at $2300 \mathrm{~K}$, and compared the measured intensity and line position with the TOTO data. In addition, Nugroho et al. (2020) applied high-resolution cross-correlation spectroscopy method using the $\mathrm{TiO}$ Plez1998 (updated version) and TOTO line lists, but they could not detect TiO signature in KELT-20b atmosphere. Note that even though both Nugroho et al. (2020) and our study have used the same TiO-TOTO line list, our generated ACS data are different from their data because of the following reasons. First, their resolution is 100 points/wavenumber, however, our resolution is at least 1 times finer than them for several pressuretemperature points. Second, Their line wing cut-off is $100 \mathrm{~cm}^{-1}$ which is different from ours. Besides, our pre-generated ACS data covers a wider temperature and pressure range with larger grid points than the current pre-generated ACSs.

\section{3. $V O$}

Numerous laboratory works have focused on determining the spectroscopic constants of the ground-state $\left(X^{4} \Sigma^{-}\right)$and low-lying excited states, including the $A-X(\sim 1.1 \mu \mathrm{m} \quad$ (Cheung et al. 1982)), $B-X($ $\sim 0.74 \mu \mathrm{m} \quad$ (Huang et al. 1992)), and $C-X(\sim 0.43-$ $0.58 \mu \mathrm{m} \quad$ (Hopkins et al. 2009)) systems as well as quartet-quartet transitions (e.g., ${ }^{4} \Delta-{ }^{4} \Pi$ and ${ }^{4} \Delta-{ }^{4} \Phi$, $\sim 0.85$ and $1.05 \mu \mathrm{m}$ (Merer et al. 1987)) and doubletdoublet transitions (e.g., ${ }^{2} \Delta-{ }^{2} \Delta$ and ${ }^{2} \Phi-{ }^{2} \Delta, \sim 0.45$ and $1.4 \mu \mathrm{m}$ (Ram et al. 2002; Ram \& Bernath 2005). Complimenting the experiments, several theoretical $a b$ initio works on $\mathrm{VO}$ have been undertaken to assign all rovibronic transitions to improve the atmospheric modeling of ultra-hot atmospheres (e.g., (Miliordos \& Mavridis 2007)). Hübner et al. (2015) computed potential energy surfaces of the electronic ground and excited states and their corresponding spectroscopic constants

\footnotetext{
11 exomol.com/data/molecules/TiO/49Ti-16O/Toto/
} 
(equilibrium radius, dissociation energies). McKemmish et al. (2016) (hereafter, VOMYT) improved upon these previous $a b$ initio results by including spin-orbit and spin-spin couplings as well as by leveraging available spectroscopic data to modify the potential energy surface to improve line positions and intensities, resulting in a robust VO line list with $2.77 \times 10^{8}$ transitions. We used this VOMYT line list ${ }^{12}$ to generate our VO crosssections given the parameters in Table 5. A sample of these cross-sections is shown for multiple temperatures and pressures in Fig. 8. In addition, Fig. 9 illustrates the difference between the VOMYT ACS data with the old ones computed from the Plez and Schwenke line lists for the full UV-Vis-IR range. The reason for these considerable differences is due to the calculated number of transitions and the computed electronic states from each line list. For example, Plez (1999) has accounted for 3.1 million lines, while VOMYT has 277 million transitions, which explains why it has a higher ACS value. The number of transitions in the Schwenke line list is much smaller than $V O M Y T$ as shown in the figure. Further details can be found in table 19 by McKemmish et al. (2016).

\section{4. $\mathrm{FeH}$}

$\mathrm{FeH}$ (iron monohydride) is a diatomic radical with a high-multiplicity ground state $\left(X^{4} \Delta\right)$, resulting in a complex electronic spectrum (see page 71 in Veillard (2012)). Several laboratory spectroscopic measurements have been performed since the 1970s to record and analyze the $\mathrm{FeH} F^{4} \Delta-X^{4} \Delta 0-0$ band (known as Wing-Ford band) (McCormack \& Oconnor 1976) as well as to characterize the infrared FeH spectra (Phillips et al. 1987a). Later, Dulick et al. (2003) used an ab initio quantum mechanic approach to calculate a new line list for several vibrational bands in the $F^{4} \Delta-X^{4} \Delta$ system, including the electronic transmission dipole moment and spectroscopic constants (e.g., rotation and vibration constants). Following their theoretical work, Wende, S. et al. (2010) used high-resolution spectroscopy to record the WingFord spectral lines in 989.8-1076.6 nm. In the same year, Hargreaves et al. (2010) used the above laboratory data to investigate the $E^{4} \Pi-A^{4} \Pi(\sim 1.58 \mu \mathrm{m})$ rovibrational spectra. Note that the band strength of the $E-A$ system calculated from this work has been problematic in several astrophysical spectral observations, further discussed in $\S 4.4 .1$, and so we did not include this $E-A$ band in generating our FeH opacities. More recently, (Bernath 2020b) synthesized these works into

12 exomol.com/data/molecules/VO/51V-16O/VOMYT/ the MoLLIST line database. We used the FeH line list by Dulick et al. (2003) ${ }^{13}$ to compute our cross-sections over the properties given in Table 5. Fig. 10 (left) illustrates a few pressure points from our grid and Fig. 10 (right) shows the results of including the Hargreaves et al. (2010) $E-A$ band.

\subsubsection{Comments on FeH Line Lists}

The history of the FeH line lists is complex and deserves an expanded discussion. Much of the uncertainty involves the $E^{4} \Pi-A^{4} \Pi$ electronic transition near $1.58 \mu \mathrm{m}\left(6300 \mathrm{~cm}^{-1}\right.$, Fig. 10 (right panel). Hargreaves et al. (2010) obtained the positions and intensities within this band using a spectrum previously recorded with a Fourier Transform Spectrometer at Kitt Peak National Observatory from an emission spectrum produced by a King-type furnace as the source (Phillips et al. 1987b). A calibration step was necessary to convert the measured emission intensities, without an absolute intensity scale, into line strengths at a given temperature, resulting in their line list for this $E-A$ transition.

However, there are several potential sources of uncertainty in this measured intensities-to-line strengths conversion. First, the temperature of the tube furnace was determined to be closer to $2200 \mathrm{~K}$ based on the relative line intensities (instead of the $2673 \mathrm{~K}$ as recorded by Phillips et al. (1987b)). The actual final calibration connecting the lab data to intensities was done using lines that had been individually identified by Balfour et al. (2004). The spread in the intensities (see Figure 1 in Hargreaves et al. (2010) paper) is quite large and a linear regression was used to obtain a conversion factor for the intensities. Hargreaves et al. (2010) mentioned in the paper that points used to calibrate the laboratory intensities contained considerable scatter, and the change in the assumed temperature may play a role in the intensity problem. In addition, An average lower state energy $\left(2250 \mathrm{~cm}^{-1}\right)$ is used for all unassigned lines ( $96 \%$ of the lines provided), which would contribute to the uncertainty at temperatures away from $2200 \mathrm{~K}$ as the line list from this work was provided at $2200 \mathrm{~K}$. Two points to note: the ratio of the partition function $Q(2673 \mathrm{~K}) / Q(2200 \mathrm{~K})=1.64$ and the change in the factor needed to convert emission intensities to absorption values (see Eq. 6 in Hargreaves et al. (2010)) is approximately a factor of 2 when considering the ratio of the two temperatures. These caveats may play a role in

\footnotetext{
13 exomol.com/data/molecules/FeH/56Fe-1H/MoLLIST/; Note that the reference given in the ExoMol website is mistakenly to the recent work by Wende, S. et al. (2010), but the provided line lists there are from Dulick et al. (2003).
} 
astrophysical data-model comparisons, suggesting that this $\mathrm{FeH}$ band, in particular, deserves further theoretical and laboratory attention.

\section{5. $\mathrm{TiH}$}

Several spectroscopic works have been performed to record and assign the TiH spectral systems including the ${ }^{4} \Gamma-X^{4} \Phi$ system (530 nm Steimle et al. (1991); Launila $\&$ Lindgren (1996)) and the vibrational ${ }^{4} \Phi-X^{4} \Phi$ system (938 nm (Andersson et al. 2003; Linton et al. 2012)) system. Burrows et al. (2005) re-investigate the previous TiH spectroscopy data in order to derive new spectroscopic constants to produce a line list, called MoLLIST. We employed this line-list ${ }^{14}$ to generate pre-computed ACS data over the parameters given in Table 5. Crosssections for select pressures are shown in Fig. 11 (left).

\section{6. $\mathrm{CrH}$}

An emission spectrum of the $A^{6} \Sigma^{+}-X^{6} \Sigma^{+}(0.74-$ $1 \mu \mathrm{m}$ ) system was first recorded by (Ram et al. 1993), later expanded upon by (Bauschlicher et al. 2001) who fit more bands (i.e., $1-0,1-1$, and $2-0$ ) in the region of $0.67-1.11 \mu \mathrm{m}$. Recently, Bernath (2020b) combined them all these line-lists into the MoLLIST database. We used MoLLIST reformatted by the ExoMol group ${ }^{15}$ to generate ACS data for the available spectral range (i.e., $0.7-1.6 \mu \mathrm{m}$ ). Fig. 11 (right) shows our cross-sections as a function of pressure.

\section{7. $\mathrm{CaH}$}

The infrared and electronic transitions of the ground state $X^{2} \Sigma^{+}$, excited states $\left(A^{2} \Pi, B^{2} \Sigma^{+}\right.$, and $E^{2} \Sigma$, $0.45-1$ and $2-14 \mu \mathrm{m}$ respectively) have been characterized using high-resolution Fourier transform spectroscopy (Ram et al. 2011; Shayesteh et al. 2013). The Einstein-A-coefficients have also been calculated for these band systems (Li et al. 2012; Alavi \& Shayesteh 2017a; Yadin et al. 2012). Additional ab initio investigations determined the transition dipole moments of the ground state $X^{2} \Sigma^{+}$, excited states (i.e., $A^{2} \Pi, B^{2} \Sigma^{+}$, and $E^{2} \Sigma$ ) (Shayesteh et al. 2017; Weck et al. 2003) covering wavelengths from 1-2 and 14-100 $\mu \mathrm{m}$. Our crosssections employs the laboratory-based lists synthesized by Bernath (2020b) ${ }^{16}$ over the $0.45-1$ and $2-14 \mu \mathrm{m}$ regions. For the remaining spectral range, i.e. 1-2 and 14-100 $\mu \mathrm{m}$, we used the ab initio line list from Yadin

\footnotetext{
14 exomol.com/data/molecules/TiH/48Ti-1H/MoLLIST/

15 exomol.com/data/molecules/CrH/52Cr-1H/MoLLIST/

16 exomol.com/data/molecules/CaH/40Ca-1H/MoLLIST
}

et al. $(2012)^{17}$. Figure 12 (right) summarizes our crosssections at $2000 \mathrm{~K}$ over a range of pressures.

\section{8. $M g H$}

The spectroscopy of the ground state and low-lying excited electronic states of $\mathrm{MgH}$ has been the subject of numerous investigations. Balfour \& Cartwright (1975a,b) and later Shayesteh \& Bernath (2011) have measured the rovibronic transitions of the $A-X$ and $B^{\prime}-X$ systems in order to extract line positions, dissociation energies, rotational constants, and their potential energy surfaces. Employing the latest potential energy surface's, Yadin et al. (2012) calculate all infrared transitions for the $\mathrm{MgH}$ ground electronic state for 1.2 $100 \mu \mathrm{m}$. In addition, GharibNezhad et al. (2013) used high-resolution spectroscopic data to produce a line list from $0.35-1.2 \mu \mathrm{m}$. We compute our cross-section using the Yadin et al. (2012) ${ }^{18}$ line list from $1.2-100 \mu \mathrm{m}$ and the GharibNezhad et al. $(2013)^{19}$ from $0.35-1.2 \mu \mathrm{m}$. Figure 12(left) illustrates a slice on our cross-section grid for $2000 \mathrm{~K}$ over a representative range in pressures.

\section{9. $\mathrm{SiO}$}

Since the 1970s, $\mathrm{SiO}$ has been the subject of several spectroscopic studies over the UV-Vis to the IR using data recorded from both hot gas cells as well as the sunspots (Barrow \& Stone 1975; Campbell et al. 1995; Sonnabend et al. 2006). Furthermore, the $\mathrm{SiO}$ potential energy surfaces, Einstein A coefficients, and other spectroscopic constants have been theoretically studied using ab inito method (Langhoff \& Bauschlicher 1993; Langhoff \& Arnold 1979). Later, as a part of the ExoMol initiative, Barton et al. (2013) improved the potential energy surface of the ground state and dipole moment function. In our calculation, we used the Barton et al. (2013) line list ${ }^{20}$ to calculate cross-sections over $1.67-100 \mu \mathrm{m}$ for the temperature and pressure range's reported in Table 5. Figure 13(right) shows a slice on our ACS grid for $2000 \mathrm{~K}$ over a representative range in pressures.

\subsection{0. $A l H$}

Different rovibronic lines of AlH have been measured in the lab using high-resolution spectroscopy, including the $A^{1} \Pi-X^{1} \Sigma^{+}$band at $400-550 \mathrm{~nm}$ (Szajna \& Zachwieja 2009; Ram \& Bernath 1996), the $C^{1} \Sigma^{+}-X^{1} \Sigma^{+}$

\footnotetext{
17 exomol.com/data/molecules/CaH/40Ca-1H/Yadin/

18 exomol.com/data/molecules/MgH/24Mg-1H/Yueqi/

19 exomol.com/data/molecules/MgH/24Mg-1H/MoLLIST

20 exomol.com/data/molecules/SiO/28Si-16O/EBJT/
} 
band at 220-240 nm (Szajna \& Zachwieja 2010). In addition, the $\mathrm{AlH}$ dissociation energy and the low-lying electronic states have been characterized using $a b$ initio approach's Matos et al. (1987). Recently, Yurchenko et al. (2018) employed these spectroscopic constants of the ground state and the first low-lying excited state, and experimental potential energy curves to calculated all rovibrational transitions between the energy levels. We employed their line list, named $W Y L L o T^{21,22}$, to generate our ACSs from 0.4-100 $\mu \mathrm{m}$ for the temperature and pressure ranges in Table 5. Figure 13 (right) represents a subset of our ACSs as a function of pressure.

\subsection{Comparison to Other Absorption Cross-Section Databases}

There are other cross-section databases, some publicly available such as (Villanueva et al. 2018; Chubb et al. 2020), and some are not (Sharp \& Burrows 2007; Freedman et al. 2008; Freedman et al. 2014; Goyal et al. 2018). We build on these past works by implementing the latest line lists, primarily drawn from the ExoMol, and provide an up-front description of our sources of pressure broadening and how we approach the problem when no data is available (see §3). Table 7 summarizes the known differences between our database and past absorption cross-section database's. In summary, our database resolution is sufficiently large to be applicable for interpretation of $H S T$ and $J W S T$ observed data as well as high-resolution cross-correlations'. In addition, we used a constant number for the wing cut-off to avoid overestimation of the opacity continuum. The temperature, pressure, and wavelength ranges are also wider and have more grids (1460 T-P points) than other available pre-generated databases in order to fully model the (Ultra)Hot-Jupiters and M-L dwarf atmospheres.

\section{IMPACT OF OPACITIES ON HIGHLY-IRRADIATED EXOPLANET ATMOSPHERES}

We assess the impact of the newly generated ACSs on the atmospheres and spectra of M dwarfs and hot Jupiters-specifically, the influence of metal hydrides and oxides from EXOPLINES vs. Freedman2014 (Freedman et al. 2014) (see the list of the Freedman2014's line lists at Table 2 by Lupu et al. (2014)). First, we simulate a representative Ultra-Hot Jupiter atmosphere $\left(\mathrm{T}_{i r r}=\right.$ $\sim 2000 \mathrm{~K}, \log g=3, \mathrm{~T}_{\text {star }}=6500 \mathrm{~K}$, solar composition)

21 exomol.com/data/molecules/AlH/27Al-1H/AlHambra/

22 Although in the paper by Yurchenko et al. (2018), it was named as WYLLoT line list, the ExoMol website provided the AlH line list files with "AlHambra" name. with a 1D-self-consistent radiative-convective thermochemical equilibrium model, Sc-CHIMERA ${ }^{23}$, previously described in Piskorz et al. (2018); Arcangeli et al. (2018); Gharib-Nezhad \& Line (2019). We compute the planetary emission and transmission spectrum from 0.3 $5.0 \mu \mathrm{m}$. Major opacity sources included in these models are $\mathrm{H}_{2} \mathrm{O}, \mathrm{TiO}, \mathrm{VO}, \mathrm{FeH}, \mathrm{CO}, \mathrm{CO}_{2}, \mathrm{CH}_{4}, \mathrm{NH}_{3}$, $\mathrm{H}_{2} \mathrm{~S}, \mathrm{HCN}, \mathrm{C}_{2} \mathrm{H}_{2}, \mathrm{PH}_{3}, \mathrm{CaH}, \mathrm{MgH}, \mathrm{CrH}, \mathrm{SiO}, \mathrm{AlH}$, $\mathrm{Na}, \mathrm{K}, \mathrm{H}_{2}-\mathrm{H}_{2}, \mathrm{H}_{2}-\mathrm{He}$, Fe, Mg, Ca, H- bound/free-free continuum, and $\mathrm{H}_{2} / \mathrm{He}$ Rayleigh scattering within the correlated-K resort-rebin framework (Amundsen et al. 2016).

We illustrate the effects of $\mathrm{TiO}, \mathrm{VO}, \mathrm{H}_{2} \mathrm{O}$ individually, and as combined with other metal hydrides $(\mathrm{FeH}$, $\mathrm{CaH}, \mathrm{MgH}, \mathrm{CrH}, \mathrm{SiO}$, and $\mathrm{AlH}$, with sources listed in Table 4). Figure 14 shows both transmission (left) and emission (right) spectra for an Ultra Hot Jupiter, varying only the choice of line list. Focusing first on the transmission spectrum, we report significant differences in the spectral shape below $1.0 \mu \mathrm{m}$; however, in the near-infrared, the residual differences are well below $50 \mathrm{ppm}^{24}$. Between $0.3-1.0 \mu \mathrm{m}$, we find that major differences are due to the choice of TiO line list (TOTO vs. Schwenke, Freedman et al. (2014)), leading up to $320 \mathrm{ppm}$ in residuals. Differences arising purely from the choice of metal hydride line lists $(\mathrm{FeH}, \mathrm{MgH}$, and $\mathrm{CaH}$ from MoLLIST), are around $75 \mathrm{ppm}$ for the transmission spectrum, notably over wavelengths covered by the HST/WFC3 bandpass $(1.4-2.0 \mu \mathrm{m})$. We also investigated the influence of $\mathrm{H}_{2} \mathrm{O}$ line list choice on the transmission spectra, where we find residual differences fall under $10 \mathrm{ppm}$ when comparing Schwenke (Freedman et al. 2014) to POKAZATEL.

For the thermal emission spectrum; similarly, TiO line list choice leads to significant differences in the spectral shape, especially from $1.0-5.0 \mu \mathrm{m}$, with up to $80 \mathrm{ppm}$ differences. Metal hydrides and $\mathrm{H}_{2} \mathrm{O}$ lead to residual differences around $10 \mathrm{ppm}$, also consistent with the transmission spectrum (see Figure 14). Moreover, the total differences between all old ACS data (e.g., TiO-Schwenke, VO-Plez, MgH-Weck, CaHWeck, FeH-old) against the EXOPLINES ACS data (TiOTOTO, VO-VOMYT, MgH-MoLLIST, CaH-MoLLIST, FeH-MoLLIST), are dominated by $\mathrm{TiO}$ and metal hydride line list differences, altogether leading up to the same level of residuals.

\footnotetext{
23 The core radiative transfer, CHIMERA, found at https://github.com/mrline/CHIMERA

${ }^{24}$ HST WFC3 $(1.1-1.4 \mu \mathrm{m})$ precision of $15-50 \mathrm{ppm}$ are routinely achieved (Line et al. 2016; Kreidberg et al. 2014). Comparable or better precision are anticipated with $J W S T$ (Bean et al. 2018)
} 
We also illustrated the impact of these new ACSs data on the Temperature-Pressure (TP-) profile of this candidate WASP-121b atmosphere in Fig. 15. This figure shows that the new TiO-TOTO ACS data change the $\mathrm{TP}$ profile by $\sim 60 \mathrm{~K}$ between $1 \mathrm{mbar}$ and 1 bar. The impact of all new molecular ACSs ( $\mathrm{TiO}, \mathrm{VO}$, etc.) is $\sim 130 \mathrm{~K}$ between 1 mbar to 1 bar. These differences in the TP-profile lead to change in the atmospheric chemical composition, emission, and transmission synthetic spectra.

More recently, Piette et al. (2020) showed that incorporating the different $\mathrm{TiO}$ opacities (i.e., TOTO, Plez, and Schwenke) biases the synthetic emission and transmission spectra of ultra-hot Jupiters $\left(\mathrm{R} \sim 10^{4}\right)$ up to $\sim 1000 \mathrm{ppm}$ in the visible and $100 \mathrm{ppm}$ in the near-IR spectral region. In another instance for high-resolution ground-based spectroscopy, Herman et al. (2020) used TiO-Plez ACS to identify the potential presence of $\mathrm{TiO}$ optical lines in WASP-33b. Implications of these works strongly vouch for the critical vetting of line lists used to compute appropriate ACS, especially concerning oxygen-bearing molecules such as $\mathrm{H}_{2} \mathrm{O}$ and $\mathrm{TiO}$ to robustly determine bulk chemical properties (such as $\mathrm{C} / \mathrm{O}$ ratio) of sub-stellar atmospheres (Molaverdikhani et al. 2019), as well as improving the atmospheric circulation models of hot Jupiters (Kataria et al. 2016).

\section{IMPACT OF OPACITIES ON M-DWARF ATMOSPHERES}

M-dwarf atmospheric spectra are also subject to differences arising from various line lists. To quantify these differences, using the same self-consistent modeling framework described above, we simulate a dustfree $T_{\text {eff }}=3000 \mathrm{~K}, \log g=5$, solar composition atmosphere. In an upcoming paper by Iyer et al. (In Prep), we will discuss our M-dwarf model in great detail. Consistent with the hot Jupiter case, TiO line-list differences have the largest influence on the spectral shape and the temperature profile. We find that the TiOTOTO (EXOPLINES) line-list alone, when compared to the Schwenke/Allard (Figure 16, left) produces spectral differences of up to $125 \%$, primarily between $0.3-1.0 \mu \mathrm{m}$. Alternatively, the effect of VO opacity (Plez (Freedman et al. 2014) vs VOMYT (EXOPLINES)) and $\mathrm{H}_{2} \mathrm{O}$ (Schwenke (Freedman et al. 2014) vs POKAZATEL (EXOPLINES)) do not contribute to any notable differences at lower resolutions $(\mathrm{R}=100)$, however, we see up to a $30 \%$ difference due to the effect of metal hydrides below $1 \mu \mathrm{m}$, and up to $12 \%$ over the near-IR $(1.4-2.0 \mu \mathrm{m})$ bandpasses. $\mathrm{TiO}$ attributes to the largest variation in the temperature profile as well; (see Figure 16, right), with up to $50 \mathrm{~K}$ in differences between 1 mbar to 0.1 bar, and up to $20 \mathrm{~K}$ difference deeper than 100 bars, because of a subtle shift in the adiabat. Furthermore, the absorption cross-sections from the hydrides (calculated from MoLLIST) lead to thermal profile differences of up to 10 and $20 \mathrm{~K}$ respectively, from 1 mbar to 0.1 bar and down to 100 bars. Undoubtedly, this level of bias - in both hot Jupiters and M-dwarfs - arising purely from generational differences of line lists would lead to improper atmospheric inferences. McKemmish et al. (2019b) also have recently investigated the impact of (Plez 1998), (Schwenke 1998), and TOTO TiO line lists on M-dwarf flux, which showed large differences in their synthetic flux.

\section{CURRENT CHALLENGES WITH OPACITIES}

Several spectroscopic parameters are critical for computing absorption cross sections and their subsequent effect in the modeling of observations (e.g., Fortney et al. 2019). Some challenges arise simply due to lack of laboratory data, and some are because of the computational shortcuts used to improve the speed of these often lengthy calculations. Therefore, it is crucial to consider these all differences between the Voigt profiles, broadening treatment, wing cut-off and other details in order to evaluate and cross-check different opacity databases generated by either CPU-based computing codes such as ExoCross and HAPI ${ }^{25}$, or GPU-based codes such as Radis ${ }^{26}$ and Helios-K ${ }^{27}$ (Yurchenko et al. 2018; Kochanov et al. 2016; van den Bekerom \& Pannier 2021; Grimm et al. 2021). We briefly discuss the current pressing challenges in producing accurate cross-sections:

- The Lack of Pressure Broadening Data-As discussed in $\S 3$, the pressure-broadening coefficients, $\gamma_{\mathrm{L}}$ and $n_{T}$, are critical to the proper computation of the Lorentz line widths and wings, which dictates the "off line" continuum. For an absorber, these coefficients depend on the broadening gas, quantum $J$ number, molecular symmetry ${ }^{28}$, temperature, and also the specific interactions with the broadening gas (e.g., the complexity in the line profile of $\mathrm{Na}$ and $\mathrm{K}$ atoms studied

\footnotetext{
25 https://github.com/hitranonline/hapi

26 https://radis.readthedocs.io/en/latest/

27 https://helios-k.readthedocs.io/en/latest/

${ }^{28}$ Spectroscopic parameters such as pressure-broadening and molecular angular momentums (simply, quantum numbers) depend on the molecular geometry and the symmetry between different energy states. For example, $\mathrm{CH}_{4}$ is a tetrahedral molecule and has $A_{1}, A_{1}, F_{1}, F_{2}$ and $E$ symmetries, which can be found from character tables. For further details, check Table 3 by Rothman et al. (2005) and the discussion in chapter 3 by Bernath (2005). Several examples of broadening data can also be found in chapters 4 and 5 by Buldyreva et al. (2011).
} 
by Allard et al. $(2019,2016))$. The current state of broadening data for many molecules important in exoplanet/substellar atmospheres is concerning. In most cases for many absorbers $\gamma_{\mathrm{L}}$ measurements are limited to below $500 \mathrm{~K}$ (e.g., see review by Hartmann et al. (2018)). Most data are only applicable to "Earth-like" conditions through "air-broadening" or reducing solarsystem giant-like atmospheres through $\mathrm{H}_{2} / \mathrm{He}$ broadening. Regarding the metal hydrides and oxides in question, as far as our best knowledge, there is no laboratory/theoretical broadening data available..

- Compositional dependency of absorption cross sections: One of the key findings of the Kepler Mission is that a majority of exoplanets fall within this "warm mini-Neptune" regime $\left(\mathrm{R}_{p} \sim 2-4 \mathrm{R}_{\text {Earth }} \mathrm{T}<1000 \mathrm{~K}\right)$ (Batalha 2014). The bulk composition of these exoplanets is not hydrogen-dominant, and therefore, incorporating the pre-generated $\mathrm{H}_{2} / \mathrm{He}$-broadened opacities to model their atmospheres may cause errors and biases in interpreting their observational emission and transmission spectra. For example, (Gharib-Nezhad \& Line 2019) have shown that using the $\mathrm{H}_{2} / \mathrm{He}-$ broadened of water opacity for modeling a candidate super-Earth steam atmosphere could lead up to 80 ppm (part-per-million) difference in transmission and $250 \mathrm{ppm}$ in the emission spectra, which are detectable by both $H S T$ and the future $J W S T$ telescopes. As a result, there is uncertainty and inaccuracy in atmospheric modeling observables such as composition, which could lead to a misinterpretation of observational data.

- Line Profile - The Voigt profile is the most common line shape applied in opacity computations. This profile is based on the ideal gas assumption where no interactions between broadening (e.g., the bath gas/background) molecules exist. However, these interactions do occur, and hence, this effect should be taken into account with more advanced line profiles such as the speed-dependent Voigt profile (Pickett 1980; Buldyreva et al. 2011). These broadener gas interactions have been explored at both low and high pressures (Ngo et al. 2012; Grigoriev et al. 1999). Table 1 by Ngo et al. (2013) illuminates some of these differences.

- Voigt Profile Algorithm-The specific algorithm and inherent approximations used to compute the Voigt profile can result in non-negligible uncertainties. The Voigt algorithm by Humlíček (1979) is the most widely used due to the balance between accuracy and computational efficiency. However, it has been shown (Zaghloul 2015; Schreier 2018a) that this algorithm is not always accurate over the pressure, temperature, and wavelength regions. Other algorithms exist, but need to be continually assessed for accuracy in different situations (Schreier 2018b).

- Line Wing Cut-Off-This dictates the frequency extent to which the Lorentz line wings are computed from the line core-typically employed to reduce the computational burden. Choosing too short of a wing cut-off, particularly for high pressures (>10 bar), can result in large errors in the opacity continuum depending upon the number of transitions. Hartmann et al. (2002) proposed an empirical correction factor for the wing cut-off to fully model the $3.3 \mu \mathrm{m}$ band of $\mathrm{CH}_{4}$, however, this study is only limited to room temperature and the broadener is $\mathrm{H}_{2}$ molecule. Further investigations are required to understand the accurate wing cut-off for different line profiles.

- Intensity Cut-Off - This effectively limits the number of lines over which to compute the line strengths and profiles, and subsequent cross sections. Line intensities are typically given as the line strength at a particular reference temperature, usually $296 \mathrm{~K}$. Choosing a cut will remove the weaker lines that can present as stronger lines at higher temperatures, resulting in a critical loss in overall opacity. The cutoff choice is particularly influential with line lists that contain billions of transitions, which can result in the exclusion of a substantial portion of the weaker transitions, resulting in an altered "pseudo-continuum" shape. Figure 17 illustrates the impact of line-strength cut-off on the POKAZ ATEL $\mathrm{H}_{2} \mathrm{O}$ cross-sections at $2000 \mathrm{~K}$, $10^{-3}$ bar over the short wavelengths $(0.25-0.5 \mu \mathrm{m})$.

- Pressure-shift - As a result of collision by the broadening gas, the line-center blue/red shifts by $\sim 0.001$ $0.005 \mathrm{~cm}^{-1} /$ bar, depending on the broadener, temperature, and quantum number $J$. At high pressures, lines extensively overlap due to their large line-width, but the line center will shift as well. To the best of our knowledge, this concept has not been applied in generating ACSs for the majority of absorbers (including the current study).

- Partition function - The strength of each individual line for different temperatures is scaled directly by the partition function, usually treated as a separate data source in opacity calculations. Partition function value for a given temperature is calculated from all the energy levels that have been obtained from spectroscopy measurements or through abinitio calculations (e.g., see equations by Barklem \& Collet (2016) and Yurchenko et al. (2018)). Hence, incompleteness 
or inaccuracy in the measured/computed energy levels results in errors in the partition function, and consequently in the computed line strengths, and resulting cross-sections. For example, the water partition function and its associated uncertainty have been studied by Vidler \& Tennyson (2000). In addition, Sousa-Silva et al. (2014) have assessed the potential errors and challenges associated with calculating the $\mathrm{PH}_{3}$ and $\mathrm{NH}_{3}$ partition function values.

\section{SUMMARY AND CONCLUSIONS}

The major objective of this investigation is to provide a comprehensive pre-computed absorption crosssection database relevant to the spectral modeling of atmospheres of irradiated hot Jupiters and substellar objects. To this end, we present a public database of pre-computed molecular absorption cross-sections for all the isotopologues of $\mathrm{MgH}, \mathrm{AlH}, \mathrm{CaH}, \mathrm{TiH}, \mathrm{CrH}, \mathrm{FeH}$, $\mathrm{SiO}, \mathrm{TiO}, \mathrm{VO}$, and $\mathrm{H}_{2} \mathrm{O}$. The pressure and temperature range of the grid span $10^{-6}-3000$ bar and $75-4000 \mathrm{~K}$, respectively (except $\mathrm{H}_{2} \mathrm{O}$ which is 500-4000 K) and their spectral coverage vary depending on the availability of the line list data, typically covering $0.25-330 \mu \mathrm{m}$ where possible.

We applied detailed care when determining the pressure broadening coefficients. Where available, we started from the published values estimated for the $\mathrm{H}_{2} / \mathrm{He}$ broadening, or otherwise used a $J$-dependent collision theory. We described the history of the line lists of various species, and when possible compared the influence of available line lists on the absorption-cross sections. Finally, we determined the impact of line list choice (hence cross-sections) on the temperature structure and emergent spectra of a hot Jupiter and M-dwarf using a 1D self-consistent radiative-convective model. From the latter experiment we found that:

- The TiO-TOTO line list compared to the TiOSchwenke line-list (and subsequent cross-sections) had the most significant influence on the thermal structures of the hot Jupiter ( $60 \mathrm{~K}$ differences $)$ and a generic $3000 \mathrm{~K} \mathrm{M}$-dwarf (50 K differences). The resultant hot Jupiter spectra $(\mathrm{R}=100)$ differed between the two line-list scenarios of up to $\sim 80 \mathrm{ppm}$ in emission/secondary eclipse and $\sim 320 \mathrm{ppm}$ (near $0.3-1 \mu \mathrm{m}$ ) in transmission. The M-dwarf emergent spectra differed up to $125 \%$ over the wavelengths most influenced by $\mathrm{TiO}(0.3-1.0 \mu \mathrm{m})$.

- Considering the large differences in the number of transitions in the various $\mathrm{H}_{2} \mathrm{O}$ line lists (see Table 6 and Fig. 3), the resulting differences in the emergent spectra and temperature structure were are negligible at these resolutions $(\mathrm{R}=100)$. However, at high resolution $(\mathrm{R}>50,000)$ these differences in line positions and intensities are more apparent and have been previously shown to influence molecular abundance determinations of transiting hot Jupiter's using crosscorrelation spectroscopy Brogi \& Line (2019); Gandhi et al. (2020).

- The metal hydride (FeH, MgH, and $\mathrm{CaH}$ ) crosssections derived from the MoLLIST line-lists compared to past line lists (MgH-Weck, CaH-Weck) also result in notable differences in atmospheric observables, with up to an $80 \mathrm{~K}$ difference in the hot Jupiter temperature structure and $10 \mathrm{~K}$ in the M-dwarf. Differences in the hot Jupiter emission/transmission spectra approach $\sim 50 \mathrm{ppm}$ (around 1.4-2 $\mu \mathrm{m}$ ). In the M-dwarf spectra, up to $45 \%$ change in flux occurs near $1.0 \mu \mathrm{m}$ and $12 \%$ between $1.4-2.0 \mu \mathrm{m}$.

With the upcoming higher fidelity characterization of extra-solar planets and their hosts (e.g., M-dwarfs), accurate absorption cross section ingredients (line lists, broadening) are becoming increasingly critical to the success of properly estimating their atmospheric properties. We encourage the community to continue to invest resources in line list validation and vetting, ab initio and laboratory measurements of key broadening coefficients, and production and curation of vetted absorption crosssection databases.

\section{SUPPLEMENTARY DATA}

The pre-generated absorption cross-sections (ACS) data from this study are uploaded in ZENODO https://zenodo.org/record/4458189. Our full isotopologue-weighted binary database is approximately $0.5 \mathrm{~Tb}$ which includes 10 molecular sets of 1460 ACS temperature-pressure data with the size of $\sim 44 \mathrm{~GB}$ per molecule. The un-weighted individual isotopologues ACS binary data are consist of 22 sets of molecular ACS data with 1460 temperature-pressure points for each and the total size of $\sim 1$ TB. The database will available in multiple formats to suit various needs. First, we will post a $5 \mathrm{~Gb}$ resampled version of the database on Zenodo, due to their file size limitations. The Zenodo database will be in the same format as the (Freedman et al. 2014) database currently used by PICASO (Batalha et al. 2019; Batalha \& Rooney 2020) ${ }^{29}$. Second, the full unsampled database (MySQL format) will be available via an FTP-like portal. Last, we will also launch a web portal so that users can visualize and

${ }^{29}$ Zenodo Opacities (Freedman et al. 2014) 
plot subsets of the data without needing to download it all.

\section{ACKNOWLEDGEMENTS}

EGN would like to thank Drs. J. Fortney, R. Lupu, I. Gordon, and R. Hargreaves for their useful comments and discussions during this study. EGN and ARI acknowledge Research Computing at Arizona State University for providing HPC resources that have contributed to the research results reported within this paper. EGN, MRL, and RF acknowledge support from HST-AR-15796. MRL and ARI acknowledge support from NASA grant NNX17AB56G. NEB and RF acknowledge support from 19-NUP2019-0011. We also acknowledge the ExoMol team for their continued production of large line lists and pertinent data critical to hightemperature atmospheric modeling. EGN's research was supported by an appointment to the NASA Postdoctoral Program at the NASA Ames Research Center, administered by Universities Space Research Association under contract with NASA. Finally, we thank the anonymous reviewers for their thoughtful comments.

\section{SOFTWARE}

We used the publicly available ExoCross code (Yurchenko et al. 2018) developed by the ExoMol group to generate the cross sections, and the CHIMERA radiative transfer and retrieval tool to produce the simulated emission/transmission spectra. 


\section{APPENDIX}

\section{A. APPENDICES}

\section{A.1. Calculating the Lorentz coefficient $\left(\gamma_{\mathrm{L}}\right)$ based on collision theory}

The interaction between a given absorber with other atoms or molecules (i.e., perturbers) induces an extra width in the recorded spectral lines. In order to understand and calculate the line width, this interaction may be treated in two ways. The first approach is the classical approximation method in which the collision between the absorber and broadener occurs through a straight trajectory. The second method, however, uses quantum mechanical theory to describe the motion of perturbers. Limitations in both methods have been discussed in detail by Baranger (1958). In this study, we used Anderson theory which deals simultaneously with the motion of perturbers and the transition between energy levels with classical and quantum mechanics (Anderson 1949). The total energy in the system is then solved through perturbation theory, and the collisional half-with of the spectral line can be represented by Eq. A1 (see Eq. 28 by Anderson (1949)):

$$
\Delta \nu_{\frac{1}{2}}=\frac{1}{2 \pi} n_{b} \bar{v}_{t h} \sigma_{c o l}
$$

where $n_{b}$ is the number density of perturbers, $\bar{v}_{t h}$ is the mean relative velocity of the gas $\left(=\sqrt{8 k_{B} T / \mu \pi}\right)$, and $\sigma_{c o l}$ is the collision cross-section in $\AA^{2}$ unit. Using the gas kinetic theory, HWHM linewidth $\Delta \nu_{1 / 2}$ (in $\mathrm{Hz} / \mathrm{Pa}$ ) may be written as:

$$
\Delta \nu_{\frac{1}{2}}=\left(\frac{8 k_{B} T}{\mu \pi}\right)^{1 / 2} \frac{1}{2 k_{B} \pi} T^{-1 / 2} \sigma_{\text {col }} p_{b}
$$

where $k_{\mathrm{B}}$ is a Boltzmann constant and $\mu$ is the reduced mass of the colliding pair in atomic mass unit $(\mu=$ $\left.M_{\text {absorber }} M_{\text {broadener }} /\left(M_{\text {absorber }}+M_{\text {broadener }}\right)\right)$, and $p_{\mathrm{b}}$ is the partial pressure of the broadening gas. Comparing Eq. A2 to Eq. 2 shows that $\Delta \nu_{\frac{1}{2}}$ is equivalent to Lorentz HWHM $\left(\Gamma_{\mathrm{L}}\right.$ ) (see Eq. 36c and its related discussion by Baranger (1958)). By converting the unit of Eq. A2 to $\mathrm{cm}^{-1} / \mathrm{atm}$ and $\mathrm{cm}^{-1} /$ bar , the Lorentz coefficient $\gamma_{\mathrm{L}}$ can be written as:

$$
\gamma_{\mathrm{L}}\left[\mathrm{cm}^{-1} / \mathrm{atm}\right]=0.0567(T \mu)^{-1 / 2} \sigma_{c o l}
$$

or,

$$
\gamma_{\mathrm{L}}\left[\mathrm{cm}^{-1} / \mathrm{bar}\right]=0.0574(T \mu)^{-1 / 2} \sigma_{c o l}
$$

in which, the temperature dependence of the $\gamma_{\mathrm{L}}(T)$ is:

$$
\gamma_{\mathrm{L}}(T)=\gamma_{\mathrm{L}}\left(T_{0}\right)\left(\frac{T}{T_{0}}\right)^{-1 / 2}
$$

Note that this equation can only provide a rough estimate of the pressure-broadening coefficients because the accuracy of the results relies on the collision cross section and also kinetic theory to provide the temperature-dependence coefficient of $-1 / 2$. 
Table 1. Available calculated/estimated Lorentz coefficient, $\gamma_{\mathrm{L}, \mathrm{b}}\left[\mathrm{cm}^{-1} / \mathrm{atm}\right]$ from the literature (for Eq. 2).

\begin{tabular}{lclll}
\hline \hline Absorber & Broadener & $\gamma_{\mathrm{L}, \mathrm{b}}(J=0)^{*}$ & $\gamma_{\mathrm{L}, \mathrm{b}}$ & Reference \\
\hline $\mathrm{H}_{2} \mathrm{O}$ & $\mathrm{H}_{2}$ & 0.09 & laboratory data & Ba17 \\
$\mathrm{H}_{2} \mathrm{O}$ & $\mathrm{He}$ & 0.02 & laboratory data & Ba17 \\
\hline $\mathrm{TiO}, \mathrm{VO}$ & $\mathrm{H}_{2}$ & 0.1 & $0.1-0.002 J_{\text {lower }}{ }^{* *}$ & $\mathrm{Sh07}$ \\
$\mathrm{TiO}, \mathrm{VO}$ & $\mathrm{He}$ & 0.06 & $0.06-0.0012 J_{\text {lower }}$ & \\
\hline $\mathrm{FeH}, \mathrm{TiH}, \mathrm{CrH}$ & $\mathrm{H}_{2}$ & 0.075 & $0.075-0.001 J_{\text {lower }}$ & Du03,Bu02,Bu05 \\
$\mathrm{FeH}, \mathrm{TiH}, \mathrm{CrH}$ & $\mathrm{He}$ & 0.045 & $0.045-0.0006 J_{\text {lower }}$ & \\
\hline \hline
\end{tabular}

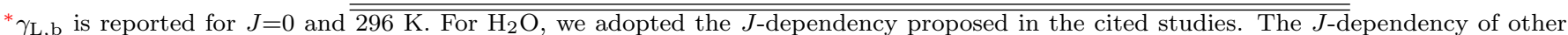
molecules is discussed in $\S 3.3$.

** Note, this equation was mistakenly proposed to provide a FWHM of $\gamma_{\mathrm{L}}$ by Sharp \& Burrows (2007), but in fact, it is for calculating the HWHM value.

References: Ba17: The $\mathrm{H}_{2} \mathrm{O}$ Lorentz coefficients were collected by the ExoMol group http://exomol.com/data/molecules/H2O/, and was discussed by Barton et al. (2017); Bu05: (Burrows et al. 2005); Bu03: (Burrows et al. 2002); Du03: (Dulick et al. 2003); Sh07: Sharp \& Burrows (2007). 
Table 2. Collision cross-section used to calculate the Lorentz pressure-broadening coefficient, $\gamma_{\mathrm{L}, \mathrm{b}}\left[\mathrm{cm}^{-1} / \mathrm{atm}\right.$

\begin{tabular}{lccccl}
\hline \hline Absorber & Broadener & $\mu$ & $\sigma_{\text {col }}$ & $\gamma_{\mathrm{L}, \mathrm{b}}$ & Reference \\
\hline${ }^{24} \mathrm{Mg}^{1} \mathrm{H}$ & $\mathrm{H}_{2}$ & 1.85 & NA & 0.08 & $=\gamma_{\mathrm{L}}\left(\mathrm{CaH}-\mathrm{H}_{2}\right)$ \\
${ }^{24} \mathrm{Mg}^{1} \mathrm{H}$ & $\mathrm{He}$ & 3.45 & NA & 0.05 & $=\gamma_{\mathrm{L}}(\mathrm{CaH}-\mathrm{He})$ \\
\hline${ }^{40} \mathrm{Ca}^{1} \mathrm{H}$ & $\mathrm{H}_{2}$ & 1.91 & NA & 0.08 & $=1.6 \gamma_{\mathrm{L}}(\mathrm{CaH}-\mathrm{He})$ \\
${ }^{40} \mathrm{Ca}^{1} \mathrm{H}$ & $\mathrm{He}$ & 3.64 & $\sim 27$ & 0.05 & {$[1]$} \\
\hline${ }^{27} \mathrm{Al}^{1} \mathrm{H}$ & $\mathrm{H}_{2}$ & 1.87 & NA & 0.08 & $=\gamma_{\mathrm{L}}\left(\mathrm{CO}-\mathrm{H}_{2}\right)$ \\
${ }^{27} \mathrm{Al}^{1} \mathrm{H}$ & $\mathrm{He}$ & 3.50 & NA & 0.05 & $=\gamma_{\mathrm{L}}(\mathrm{CO}-\mathrm{He})$ \\
\hline${ }^{28} \mathrm{Si}^{16} \mathrm{O}$ & $\mathrm{H}_{2}$ & 1.91 & $\sim 20$ & 0.05 & {$[2]$} \\
${ }^{28} \mathrm{Si}^{16} \mathrm{O}$ & $\mathrm{He}$ & 3.67 & $\sim 10$ & 0.02 & {$[3]$} \\
\hline
\end{tabular}

Notes:

1. The reported $\sigma_{\mathrm{col}}\left[\AA^{2}\right]$ is the excitation collision cross section (for $J=0 \rightarrow 1$ in most cases) for collision kinetic energy calculated for $296 \mathrm{~K}\left(E_{\text {kin }}=\left(4 k_{B} T / \pi\right) \approx 5.2 \times 10^{-21} \mathrm{Joule}=262 \mathrm{~cm}^{-1}=0.032 \mathrm{eV}\right)$.

2. The calculated $\gamma_{\mathrm{L}, \mathrm{b}}$ is used to calculate HWHM Lorentz width, $\Gamma_{\mathrm{L}, \mathrm{b}}$ in Eq. 2.

3. $\gamma_{\mathrm{L}, \mathrm{b}}$ is reported at temperature $296 \mathrm{~K}$ for $J=0$. $\S 3.3$ discusses its $J$-dependency.

4. In the case of $\mathrm{AlH}-\mathrm{H}_{2}$ and $\mathrm{AlH}-\mathrm{He}$ systems, we used $\mathrm{CO}$ Lorentz coefficients because they both have a very close dipole moment and reduced mass.

5. In a case of $\mathrm{MgH}, \mathrm{CaH}$, and $\mathrm{SiO}$ molecules, we used this assumption of $\gamma_{\mathrm{L}, \mathrm{b}}(\mathrm{He})=0.6 \gamma_{\mathrm{L}, \mathrm{b}}\left(\mathrm{H}_{2}\right)$.

Reference: [1](Akpinar \& Akbalik 2010), [2](Yang et al. 2018), [3](Dayou, F. \& Balança, C. 2006). 
Table 3. Fitted coefficients of the Fourth-order Padé formula (see Eq. 4) to provide $\gamma_{\mathrm{L}}\left[\mathrm{cm}^{-1} /\right.$ atm $]$ for $J$ quantum numbers up to 500 .

\begin{tabular}{|c|c|c|c|c|c|c|c|c|c|}
\hline Absorber & Broadener & $\mathrm{a}_{0}$ & $\mathrm{a}_{1}$ & $\mathrm{a}_{2}$ & $a_{3}$ & $\mathrm{~b}_{1}$ & $\mathrm{~b}_{2}$ & $\mathrm{~b}_{3}$ & $\mathrm{~b}_{4}$ \\
\hline $\mathrm{AlH}$ & $\mathrm{H}_{2}$ & $7.6101 \mathrm{e}-02$ & $-4.3376 \mathrm{e}-02$ & $1.9967 \mathrm{e}-02$ & $2.4755 \mathrm{e}-03$ & $-5.6857 \mathrm{e}-01$ & $2.7436 \mathrm{e}-01$ & $3.6216 \mathrm{e}-02$ & $1.5350 \mathrm{e}-05$ \\
\hline $\mathrm{AlH}$ & $\mathrm{He}$ & $4.8630 \mathrm{e}-02$ & $2.1731 \mathrm{e}+03$ & $-2.5351 \mathrm{e}+02$ & $3.8607 \mathrm{e}+01$ & $4.4644 \mathrm{e}+04$ & $-4.4438 \mathrm{e}+03$ & $6.9659 \mathrm{e}+02$ & $4.7331 \mathrm{e}+00$ \\
\hline $\mathrm{CaH}, \mathrm{MgH}$ & $\mathrm{H}_{2}$ & $8.4022 \mathrm{e}-02$ & $-8.2171 \mathrm{e}+03$ & $4.6171 \mathrm{e}+02$ & $-7.9708 \mathrm{e}+00$ & $-9.7733 e+04$ & $-1.4141 \mathrm{e}+03$ & $2.0290 \mathrm{e}+02$ & $-1.2797 \mathrm{e}+01$ \\
\hline $\mathrm{CaH}, \mathrm{MgH}$ & $\mathrm{He}$ & $5.0424 \mathrm{e}-02$ & $-1.1014 \mathrm{e}+02$ & $2.2833 \mathrm{e}+01$ & $2.8527 \mathrm{e}-01$ & $-2.4407 \mathrm{e}+03$ & $5.5472 \mathrm{e}+02$ & $-3.4490 \mathrm{e}+01$ & $6.5808 \mathrm{e}+00$ \\
\hline $\mathrm{CrH}, \mathrm{FeH}, \mathrm{TiH}$ & $\mathrm{H}_{2}$ & $7.0910 \mathrm{e}-02$ & $-6.5083 e+04$ & $2.5980 \mathrm{e}+03$ & $-3.3292 \mathrm{e}+01$ & $-9.0722 \mathrm{e}+05$ & $-4.3668 \mathrm{e}+03$ & $6.1772 \mathrm{e}+02$ & $-2.4038 \mathrm{e}+01$ \\
\hline $\mathrm{CrH}, \mathrm{FeH}, \mathrm{TiH}$ & $\mathrm{He}$ & $4.2546 \mathrm{e}-02$ & $-3.0981 \mathrm{e}+04$ & $1.2367 \mathrm{e}+03$ & $-1.5848 \mathrm{e}+01$ & $-7.1977 \mathrm{e}+05$ & $-3.4645 \mathrm{e}+03$ & $4.9008 \mathrm{e}+02$ & $-1.9071 \mathrm{e}+01$ \\
\hline $\mathrm{SiO}$ & $\mathrm{H}_{2}$ & $4.7273 \mathrm{e}-02$ & $-2.7597 \mathrm{e}+04$ & $1.1016 \mathrm{e}+03$ & $-1.4117 \mathrm{e}+01$ & $-5.7703 e+05$ & $-2.7774 \mathrm{e}+03$ & $3.9289 \mathrm{e}+02$ & $-1.5289 \mathrm{e}+01$ \\
\hline $\mathrm{SiO}$ & $\mathrm{He}$ & $2.8364 \mathrm{e}-02$ & $-6.7705 e+03$ & $2.7027 \mathrm{e}+02$ & $-3.4634 \mathrm{e}+00$ & $-2.3594 \mathrm{e}+05$ & $-1.1357 \mathrm{e}+03$ & $1.6065 \mathrm{e}+02$ & $-6.2516 \mathrm{e}+00$ \\
\hline $\mathrm{TiO}, \mathrm{VO}$ & $\mathrm{H}_{2}$ & $1.0000 \mathrm{e}-01$ & $-2.4549 \mathrm{e}+05$ & $8.7760 \mathrm{e}+03$ & $-8.7104 e+01$ & $-2.3874 \mathrm{e}+06$ & $1.6350 \mathrm{e}+04$ & $1.7569 \mathrm{e}+03$ & $-4.1520 \mathrm{e}+01$ \\
\hline $\mathrm{TiO}, \mathrm{VO}$ & $\mathrm{He}$ & $4.0000 \mathrm{e}-02$ & $-2.8682 \mathrm{e}+04$ & $1.0254 \mathrm{e}+03$ & $-1.0177 \mathrm{e}+01$ & $-6.9735 \mathrm{e}+05$ & $4.7758 \mathrm{e}+03$ & $5.1317 \mathrm{e}+02$ & $-1.2128 \mathrm{e}+01$ \\
\hline
\end{tabular}

Notes:

1. See $\S 3.3$ for a detailed discussion regarding the calculation of these Fourth-order Padé coefficients.

2. These coefficients were used to compute the pre-generated absorption cross-sections in this study. 
Table 4. Summary list of opacities: molecules, temperature, pressures, and their line lists (see $§ 3.5 \& 4)$.

\begin{tabular}{|c|c|c|c|c|c|c|c|c|c|}
\hline Absorber & Line list & $\lambda[\mu \mathrm{m}]$ & No. Lines & $\mathrm{P}$ [bar] & $\mathrm{T}[\mathrm{K}]$ & PT points & $\mathrm{I}_{\text {cutoff }}$ & Abundance $(\%)$ & Reference \\
\hline${ }^{26} \mathrm{Al}^{1} \mathrm{H}$ & WYLLoT & $0.4-330$ & $3.6 \times 10^{4}$ & $10^{-6}-3000$ & $75-4000$ & 1460 & $10^{-50}$ & 0.00 & Yu18 \\
\hline${ }^{27} \mathrm{Al}^{1} \mathrm{H}$ & WYLLoT & $0.4-330$ & $3.6 \times 10^{4}$ & $10^{-6}-3000$ & $75-4000$ & 1460 & $10^{-50}$ & 100.00 & Yu18 \\
\hline${ }^{27} \mathrm{Al}^{2} \mathrm{H}$ & WYLLoT & $0.4-330$ & $3.6 \times 10^{4}$ & $10^{-6}-3000$ & $75-4000$ & 1460 & $10^{-50}$ & 0.00 & Yu18 \\
\hline${ }^{40} \mathrm{Ca}^{1} \mathrm{H}$ & MoLLIST & $0.45-1,2-14$ & $1.9 \times 10^{4}$ & $10^{-6}-3000$ & $75-4000$ & 1460 & $10^{-50}$ & 96.93 & Al17,Li12 \\
\hline${ }^{40} \mathrm{Ca}^{1} \mathrm{H}$ & Yadin & $1-2,14-330$ & $2.7 \times 10^{4}$ & $10^{-6}-3000$ & $75-4000$ & 1460 & $10^{-50}$ & 96.93 & Ya12 \\
\hline${ }^{52} \mathrm{Cr}^{1} \mathrm{H}$ & MoLLIST & $0.7-1.6$ & $1.4 \times 10^{4}$ & $10^{-6}-3000$ & $75-4000$ & 1460 & $10^{-50}$ & 83.78 & $\mathrm{Bu} 02$ \\
\hline${ }^{56} \mathrm{Fe}^{1} \mathrm{H}$ & MoLLIST & $0.37-330$ & $1.2 \times 10^{5}$ & $10^{-6}-3000$ & $75-4000$ & 1460 & $10^{-50}$ & 91.74 & We10 \\
\hline${ }^{24} \mathrm{Mg}^{1} \mathrm{H}$ & MoLLIST & $0.35-1.2$ & $3.1 \times 10^{4}$ & $10^{-6}-3000$ & $75-4000$ & 1460 & $10^{-50}$ & 78.99 & Gh13 \\
\hline${ }^{24} \mathrm{Mg}^{1} \mathrm{H}$ & Yadin & $1.2-330$ & $6.7 \times 10^{3}$ & $10^{-6}-3000$ & $75-4000$ & 1460 & $10^{-50}$ & 78.99 & Ya12 \\
\hline${ }^{25} \mathrm{Mg}^{1} \mathrm{H}$ & Yadin & $1.2-330$ & $6.7 \times 10^{3}$ & $10^{-6}-3000$ & $75-4000$ & 1460 & $10^{-50}$ & 10.00 & Ya12 \\
\hline${ }^{26} \mathrm{Mg}^{1} \mathrm{H}$ & Yadin & $1.2-330$ & $6.7 \times 10^{3}$ & $10^{-6}-3000$ & $75-4000$ & 1460 & $10^{-50}$ & 11.01 & Ya12 \\
\hline${ }^{28} \mathrm{Si}^{16} \mathrm{O}$ & EBJT & $1.67-330$ & $1.8 \times 10^{6}$ & $10^{-6}-3000$ & $75-4000$ & 1460 & $10^{-50}$ & 92.00 & Ba13 \\
\hline${ }^{28} \mathrm{Si}^{17} \mathrm{O}$ & EBJT & $1.67-330$ & $1.8 \times 10^{6}$ & $10^{-6}-3000$ & $75-4000$ & 1460 & $10^{-50}$ & 0.03 & $\mathrm{Ba} 13$ \\
\hline${ }^{28} \mathrm{Si}^{18} \mathrm{O}$ & EBJT & $1.67-330$ & $1.8 \times 10^{6}$ & $10^{-6}-3000$ & $75-4000$ & 1460 & $10^{-50}$ & 0.19 & $\mathrm{Ba} 13$ \\
\hline${ }^{29} \mathrm{Si}^{16} \mathrm{O}$ & EBJT & $1.67-330$ & $1.8 \times 10^{6}$ & $10^{-6}-3000$ & $75-4000$ & 1460 & $10^{-50}$ & 4.67 & Ba13 \\
\hline${ }^{30} \mathrm{Si}^{16} \mathrm{O}$ & EBJT & $1.67-330$ & $1.8 \times 10^{6}$ & $10^{-6}-3000$ & $75-4000$ & 1460 & $10^{-50}$ & 3.08 & Ba13 \\
\hline${ }^{48} \mathrm{Ti}^{1} \mathrm{H}$ & MoLLIST & $0.4-2$ & $2.0 \times 10^{5}$ & $10^{-6}-3000$ & $75-4000$ & 1460 & $10^{-50}$ & 73.71 & $\mathrm{Bu} 05$ \\
\hline${ }^{46} \mathrm{Ti}^{16} \mathrm{O}$ & TOTO & $0.3-330$ & $3.0 \times 10^{7}$ & $10^{-6}-3000$ & $75-4000$ & 1460 & $10^{-50}$ & 8.22 & Mc19 \\
\hline${ }^{47} \mathrm{Ti}^{16} \mathrm{O}$ & тото & $0.3-330$ & $3.0 \times 10^{7}$ & $10^{-6}-3000$ & $75-4000$ & 1460 & $10^{-50}$ & 7.42 & Mc19 \\
\hline${ }^{48} \mathrm{Ti}^{16} \mathrm{O}$ & TOTO & $0.3-330$ & $3.0 \times 10^{7}$ & $10^{-6}-3000$ & $75-4000$ & 1460 & $10^{-50}$ & 73.54 & Mc19 \\
\hline${ }^{49} \mathrm{Ti}^{16} \mathrm{O}$ & тото & $0.3-330$ & $3.0 \times 10^{7}$ & $10^{-6}-3000$ & $75-4000$ & 1460 & $10^{-50}$ & 5.40 & Mc19 \\
\hline${ }^{50} \mathrm{Ti}^{16} \mathrm{O}$ & тото & $0.3-330$ & $3.0 \times 10^{7}$ & $10^{-6}-3000$ & $75-4000$ & 1460 & $10^{-50}$ & 5.17 & Mc19 \\
\hline${ }^{51} \mathrm{~V}^{16} \mathrm{O}$ & VOMYT & $0.28-330$ & $2.8 \times 10^{8}$ & $10^{-6}-3000$ & $75-4000$ & 1460 & $10^{-50}$ & 99.5 & Mc16 \\
\hline${ }^{1} \mathrm{H}_{2}^{16} \mathrm{O}$ & POKAZATEL & $0.25-100$ & $1.1 \times 10^{9}$ & $10^{-6}-300$ & $500-4000$ & 1460 & $10^{-34}$ & 99.75 & Po18 \\
\hline
\end{tabular}

Notes:

1. We used a single line list for some molecules such as $\mathrm{TiO}, \mathrm{SiO}$, and $\mathrm{H}_{2} \mathrm{O}$. For some molecules such as CaH and $\mathrm{MgH}$, multiple line lists were used in order to compute opacity data for the full spectral range. See $\S 3.5$ and $\S 4$ for more details on the line lists.

2. Species are weighted according to their solar/natural elemental abundances provided by NIST database: www.nist.gov/pml/atomic-weights-andisotopic-compositions-relative-atomic-masses.

3. For each species, absorption cross section data are provided in two ways: separate for each isotopologues and weighted based on their natural abundance.

References: Al17=Alavi \& Shayesteh (2017b), Bu02=Burrows et al. (2002) Ba13=Barton et al. (2013), Bu05=Burrows et al. (2005), Gh13=GharibNezhad et al. (2013), Li12=Li et al. (2012), Mc19=McKemmish et al. (2019b), Mc16=(McKemmish et al. 2016), Po18=Polyansky et al. (2018), We10=Wende, S. et al. (2010), Ya12=Yadin et al. (2012), Yu18=Yurchenko et al. (2018) 
GHARIB-NEZHAD ET AL.

Table 5. Opacity computational details

\begin{tabular}{|c|c|c|c|c|c|c|c|}
\hline \multicolumn{8}{|c|}{ Absorbers: } \\
\hline $\mathrm{H}_{2} \mathrm{O}$ & $\mathrm{TiO}$ & VO & $\mathrm{SiO}$ & \multicolumn{4}{|l|}{$\mathrm{CaH}$} \\
\hline $\mathrm{MgH}$ & $\mathrm{AlH}$ & $\mathrm{TiH}$ & $\mathrm{CrH}$ & \multicolumn{4}{|l|}{$\mathrm{FeH}$} \\
\hline \multicolumn{8}{|c|}{ Broadeners* : $85 \% \mathrm{H}_{2}+15 \% \mathrm{He}$} \\
\hline \multicolumn{8}{|c|}{$\mathbf{T}[\mathrm{K}]$ grid: $(73 \text { points })^{* *}$} \\
\hline 75 & 100 & 110 & 120 & 130 & 140 & 150 & 160 \\
\hline 170 & 180 & 190 & 200 & 210 & 220 & 230 & 240 \\
\hline 250 & 260 & 270 & 275 & 280 & 290 & 300 & 310 \\
\hline 320 & 330 & 340 & 350 & 375 & 400 & 425 & 450 \\
\hline 475 & 500 & 525 & 550 & 575 & 600 & 650 & 700 \\
\hline 750 & 800 & 850 & 900 & 950 & 1000 & 1100 & 1200 \\
\hline 1300 & 1400 & 1500 & 1600 & 1700 & 1800 & 1900 & 2000 \\
\hline 2100 & 2200 & 2300 & 2400 & 2500 & 2600 & 2700 & 2800 \\
\hline 2900 & 3000 & 3100 & 3200 & 3300 & 3400 & 3500 & 3750 \\
\hline 4000 & & & & & & & \\
\hline \multicolumn{8}{|c|}{ P [bar $]$ grid: (20 points $)$} \\
\hline $10^{-6}$ & $3 \times 10^{-6}$ & $10^{-5}$ & $3 \times 10^{-5}$ & $10^{-4}$ & $3 \times 10^{-4}$ & & \\
\hline $10^{-3}$ & $3 \times 10^{-3}$ & $10^{-1}$ & $3 \times 10^{-1}$ & $10^{-2}$ & $3 \times 10^{-2}$ & & \\
\hline 1 & 3 & 10 & 30 & 100 & 300 & & \\
\hline 1000 & 3000 & & & & & & \\
\hline
\end{tabular}

Wing cutoff:

P>200 bar: $150 \mathrm{~cm}^{-1}$

$\mathrm{P} \leqslant 200$ bar: $30 \mathrm{~cm}^{-1}$

Opacity code: ExoCross(Yurchenko et al. 2018)

Voigt Algorithm: Humlíček (1979)

* These broadening mixture is a good choice for hydrogen-dominated atmospheres such as (Ultra) hot-Jupiters and $M$ dwarfs. For high metallicity atmospheres such as super-Earths and mini-Neptunes, better choice of broadeners such as $\mathrm{H}_{2} \mathrm{O}$ and $\mathrm{CO}_{2}$ is essential.

** For $\mathrm{H}_{2} \mathrm{O}$ molecule, absorption cross-sections are calculated for temperature range 500-4000K.

$\dagger$ To compute the line-profile and include the effect of pressurebroadening, Lorentz profile is used. The calculation of Lorentz profile for each individual line can be extended to the full spectral range or be limited to a few wavenumbers. This is one main challenge in computing opacities, and some studies such as (Hartmann et al. 2002) and (Bézard et al. 2011) have shown the non-Lorentzian behavior of the line profile. See $\S 7$ for more discussion. 
Table 6. Comparing different $\mathrm{H}_{2} \mathrm{O}$ line lists.

\begin{tabular}{lcccclcl}
\hline Line Lists & Method & $\lambda[\mu \mathrm{m}]$ & $J_{\max }$ & No. Lines & Note & Ref. \\
\hline \hline PS97 & ab initio & $0.4-100$ & 55 & $\sim 3 \times 10^{8}$ & more accurate than BT2 for $\lambda>1$ & {$[1]$} \\
\hline BT2 & $a b$ initio & $0.33-100$ & 50 & $\sim 5 \times 10^{8}$ & more transition than Ames1997, shorter wavelength & {$[2]$} \\
\hline POKAZATEL & $a b$ initio & $0.25-100$ & 72 & $\sim 1 \times 10^{9}$ & higher intensity than BT2 at $\lambda<1 \mu$ m & {$[3]$} \\
\hline HITEMP & lab/ab initio & $0.33-100$ & 50 & $\sim 1 \times 10^{8}$ & included strong BT2 lines and experimental lines* & {$[4]$} \\
\hline
\end{tabular}

*After rigorous assessment of the impact of the BT2 intensity at 296-4000K, Rothman et al. (2010) have kept only $25 \%$ of the BT2 lines (see their Eq. 2 and their criteria for different wavenumber ranges and temperatures).

Ref.: [1] (Partridge \& Schwenke 1997), [2] (Barber et al. 2006), [3] (Polyansky et al. 2018), [4] (Rothman et al. 2010) 
Table 7. Summary of the current opacity databases for the molecules in our investigation.

\begin{tabular}{|c|c|c|c|c|c|c|c|}
\hline Abs. Cross Sec. & Linelist & $\lambda[\mu \mathrm{m}]$ & $\mathrm{T}[\mathrm{K}]$ & $\mathrm{P}[\mathrm{bar}]$ & Points $/ \mathrm{cm}^{-1}$ & P-T Pairs & Wing cut-off $\left[\mathrm{cm}^{-1}\right]$ \\
\hline \multicolumn{8}{|l|}{$\mathrm{H} 2 \mathrm{O}$} \\
\hline EXOPLINES & POKAZATEL & $0.25-100$ & $500-4000$ & $10^{-6}-300$ & $50-120$ & 486 & $100-300$ \\
\hline Gandhi2020* & POKAZATEL & $0.95-5$ & $400-1600$ & $10^{-5}-10$ & 100 & 49 & Not reported \\
\hline Malik2019** & $\mathrm{BT} 2$ & $0.5-20$ & $50-2900$ & $10^{-6}-10^{3}$ & 100 & 812 & 100 \\
\hline Goyal2018 ${ }^{\dagger}$ & $\mathrm{BT} 2$ & $0.33-100$ & $70-3000$ & $10^{-9}-10^{3}$ & 1000 & 800 & 100 \\
\hline MacDonald2019 & POKAZATEL & $0.4-50$ & $100-3500$ & $10^{-6}-10^{2}$ & 100 & 162 & 30 \\
\hline Freedman2014 ${ }^{\dagger \dagger}$ & Schwenke & $0.5-100$ & $75-4000$ & $10^{-6}-300$ & $80-120$ & 1060 & $25-250$ \\
\hline Chubb2020 & POKAZATEL & $0.3-50$ & $100-3400$ & $10^{-5}-100$ & $2-75$ & 594 & $10-500$ \\
\hline \multicolumn{8}{|l|}{$\mathrm{TiO}$} \\
\hline EXOPLINES & ТОто & $0.3-100$ & $75-4000$ & $10^{-6}-3000$ & $2-285$ & 1460 & 30,150 \\
\hline Malik2019 & Plez/Schwenke & $0.5-5$ & $50-2900$ & $10^{-6}-10^{3}$ & 100 & 812 & 100 \\
\hline Goyal2018 & Plez & $0.3-100$ & $70-3000$ & $10^{-9}-10^{3}$ & 1000 & 800 & 100 \\
\hline MacDonald2019 & тото & $0.4-50$ & $100-3500$ & $10^{-6}-10^{2}$ & 100 & 162 & 30 \\
\hline Freedman2014 & Schwenke/Allard & $0.32-100$ & $75-4000$ & $10^{-6}-300$ & $80-120$ & 1060 & $25-250$ \\
\hline Chubb2020 & тото & $0.3-50$ & $100-3400$ & $10^{-5}-100$ & $2-75$ & 594 & $10-500$ \\
\hline \multicolumn{8}{|l|}{ VO } \\
\hline EXOPLINES & VOMYT & $0.28-100$ & $75-4000$ & $10^{-6}-3000$ & $2-285$ & 1460 & 30,150 \\
\hline Malik2019 & VOMYT & $0.5-20$ & $50-2900$ & $10^{-6}-10^{3}$ & 100 & 812 & 100 \\
\hline Goyal2018 & VOMYT & $0.3-100$ & $70-3000$ & $10^{-9}-10^{3}$ & 1000 & 800 & 100 \\
\hline MacDonald2019 & VOMYT & $0.4-50$ & $100-3500$ & $10^{-6}-10^{2}$ & 100 & 162 & 30 \\
\hline Freedman2014 & Plez & $0.8-100$ & $75-4000$ & $10^{-6}-300$ & $80-120$ & 1060 & $25-250$ \\
\hline Chubb2020 & VOMYT & $0.3-50$ & $100-3400$ & $10^{-5}-100$ & $2-75$ & 594 & $10-500$ \\
\hline \multicolumn{8}{|l|}{$\mathrm{FeH}$} \\
\hline EXOPLINES & Dulick & $0.37-100$ & $75-4000$ & $10^{-6}-3000$ & $2-285$ & 1460 & 30,150 \\
\hline Goyal2018 & Wende2010 & $0.67-50$ & $70-3000$ & $10^{-9}-10^{3}$ & 1000 & 800 & 10 \\
\hline MacDonald2019 & Wende2010 & $0.4-50$ & $100-3500$ & $10^{-6}-10^{2}$ & 100 & 162 & 30 \\
\hline Freedman2014 & Dulick/Hargreaves & $0.37-100$ & $75-4000$ & $10^{-6}-300$ & $80-120$ & 1060 & $25-250$ \\
\hline Chubb2020 & Wende 2010 & $0.3-50$ & $100-3400$ & $10^{-5}-100$ & $2-75$ & 594 & $10-500$ \\
\hline \multicolumn{8}{|l|}{ MgH } \\
\hline EXOPLINES & MoLLIST/Yadin & $0.35-100$ & $75-4000$ & $10^{-6}-3000$ & $2-285$ & 1460 & 30,150 \\
\hline Malik2019 & Yadin & $1.2-20$ & $50-2900$ & $10^{-6}-10^{3}$ & 100 & 812 & 100 \\
\hline MacDonald2019 & MoLLIST/Yadin & $0.4-50$ & $100-3500$ & $10^{-6}-10^{2}$ & 100 & 162 & 30 \\
\hline Freedman2014 & Weck & $0.3-2.5$ & $75-4000$ & $10^{-6}-300$ & $80-120$ & 1060 & $25-250$ \\
\hline Chubb2020 & Wende2010 & $0.3-50$ & $100-3400$ & $10^{-5}-100$ & $2-75$ & 594 & $10-500$ \\
\hline \multicolumn{8}{|l|}{ AlH } \\
\hline EXOPLINES & WYLLoT & $0.4-100$ & $75-4000$ & $10^{-6}-3000$ & $2-285$ & 1460 & 30,150 \\
\hline Malik2019 & WYLLoT & $0.4-20$ & $50-2900$ & $10^{-6}-10^{3}$ & 100 & 812 & 100 \\
\hline MacDonald2019 & WYLLoT & $0.4-50$ & $100-3500$ & $10^{-6}-10^{2}$ & 100 & 162 & 30 \\
\hline Chubb2020 & WYLLoT & $0.3-50$ & $100-3400$ & $10^{-5}-100$ & $2-75$ & 594 & $10-500$ \\
\hline \multicolumn{8}{|l|}{$\mathrm{CaH}$} \\
\hline EXOPLINES & MoLLIST/Yadin & $0.45-100$ & $75-4000$ & $10^{-6}-3000$ & $2-285$ & 1460 & 30,150 \\
\hline Malik2019 & Yadin & $1-20$ & $50-2900$ & $10^{-6}-10^{3}$ & 100 & 812 & 100 \\
\hline MacDonald2019 & Yadin & $1-50$ & $100-3500$ & $10^{-6}-10^{2}$ & 100 & 162 & 30 \\
\hline Freedman2014 & Weck & $0.8-2.5$ & $75-4000$ & $10^{-6}-300$ & $80-120$ & 1060 & $25-250$ \\
\hline Chubb2020 & MoLLIST & $0.3-50$ & $100-3400$ & $10^{-5}-100$ & $2-75$ & 594 & $10-500$ \\
\hline
\end{tabular}

* Gandhi2020=Gandhi et al. (2020) ACSs data were generate to be used for calculating cross-correlation function for high-resolution exoplanet spectroscopy.

** Malik2019=Malik et al. (2019) ACS data have been used to model the irradiated exoplanets. The pressure grid used for the opacities goes from $10^{-6}$ bar to $10^{3}$ bar with a logarithmic step size of $1 / 3$ dex, that makes 28 pressure points. The temperature grid goes from $50 \mathrm{~K}$ to $2900 \mathrm{~K}$ with a linear step size of $50 \mathrm{~K}$ between $50 \mathrm{~K}$ and $700 \mathrm{~K}$, a step size of $100 \mathrm{~K}$ between $700 \mathrm{~K}$ and $1500 \mathrm{~K}$, and a step size of $200 \mathrm{~K}$ between $1500 \mathrm{~K}$ and $2900 \mathrm{~K}$. That is 29 temperature points in total. Opacities above $3000 \mathrm{~K}$ were extrapolate.

${ }^{\dagger}$ Goyal2018=Goyal et al. (2018) ACS data have been used in ATMO code for modeling forward exoplanet spectra.

${ }^{\ddagger}$ MacDonald2019=MacDonald (2019) (see chapter 5) ACS data are a part of POSIEDON database for atmospheric retrievals.

${ }^{\dagger \dagger}$ Freedman2014=(Lupu et al. 2014; Freedman et al. 2008; Freedman et al. 2014) were used in Exo-Transmit and PICASO code (Kempton et al. 2017; Batalha et al. 2019) to model transmission, emission, and reflected exoplanetary atmospheric spectra.

$\ddagger \ddagger$ Chubb2020 $=$ Chubb et al. (2020): In this work, the resolving power is 15,000 and the wing cut-off is $500 \times \gamma_{\text {Voigt }}$. In comparison, our resolution is 2-20 times larger in order to fully and accurately could be employed in modeling $J W S T$ - and high-resolution cross-correlation technique. Our wing cut-off is also a constant number of 30 and $150 \mathrm{~cm}^{-1}$ because the real shape of Voigt profile is non-Lorentzian and using a very large number can lead to an intensive overestimation of the opacity continuum. 


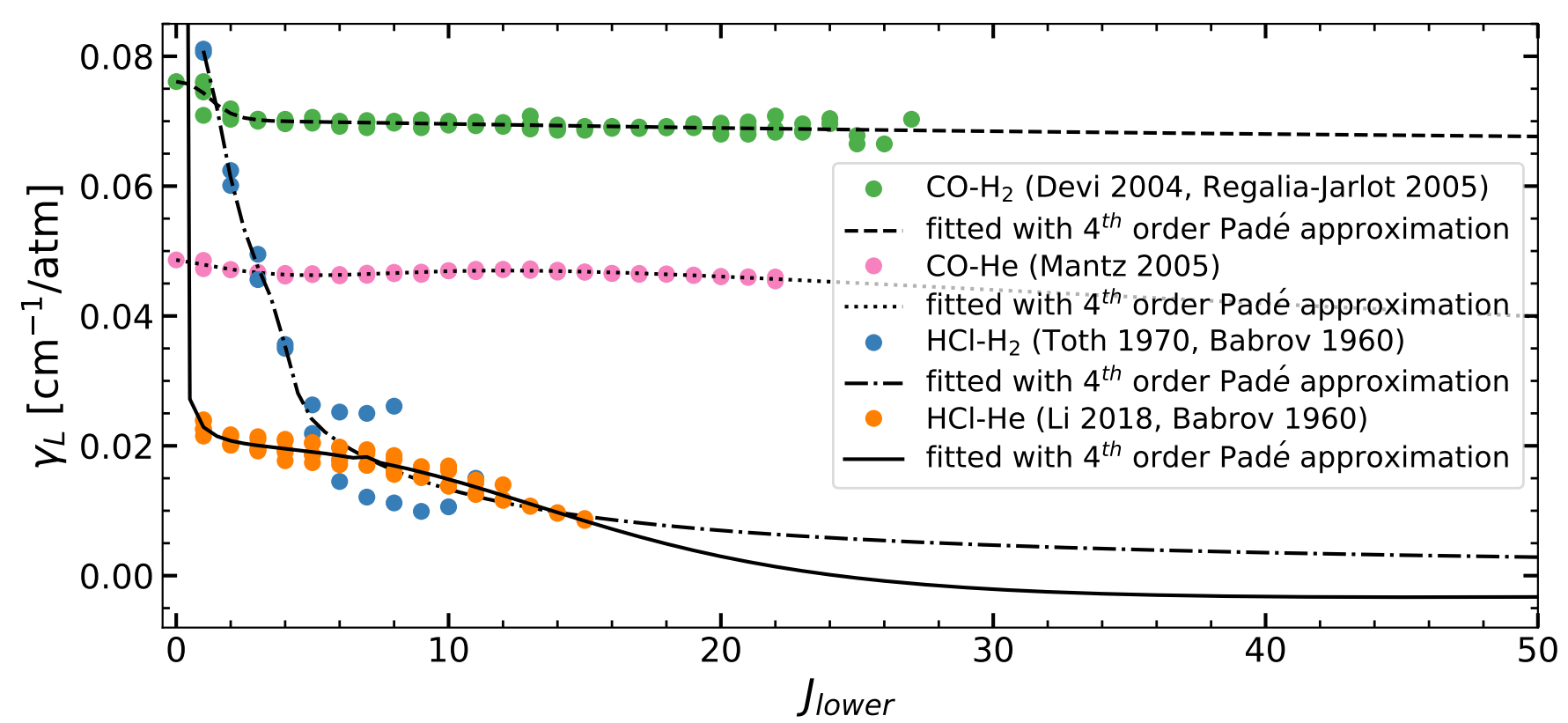

Figure 1. Comparison of $\mathrm{H}_{2}$ and He-broadening Lorentz $\mathrm{HWHM}$ of $\mathrm{CO}$ and $\mathrm{HCl}$ molecules using laboratory data. $\mathrm{HCl}-\mathrm{H}_{2}$ system has much stronger $J$-dependency than $\mathrm{CO}-\mathrm{H}_{2}$, which could be due to their difference in their dipole moments. In contrast, the $J$-dependency of He is lower in both cases. The black lines are also showing the fitted data with $4^{\text {th }}$ order Padé equation (Eq. 4). Since this equation could go to negative values in some systems and a very large number for $J=0$ (such as $\mathrm{HCl}-\mathrm{He}$ case), we applied additional limitation (see Eq. 5). We used Eq. 4 to calculate the $J$-dependency of AlH, $\mathrm{MgH}, \mathrm{CaH}$, $\mathrm{SiO}, \mathrm{TiH}, \mathrm{FeH}$, and $\mathrm{CrH}$ (see Fig. 2). Data of CO and HCl systems in this plot is collected from (Devi et al. 2004; Régalia-Jarlot et al. 2005; Mantz et al. 2005; Toth et al. 1970; Li et al. 2018; Babrov et al. 1960) (see §3.3 for more details.). 


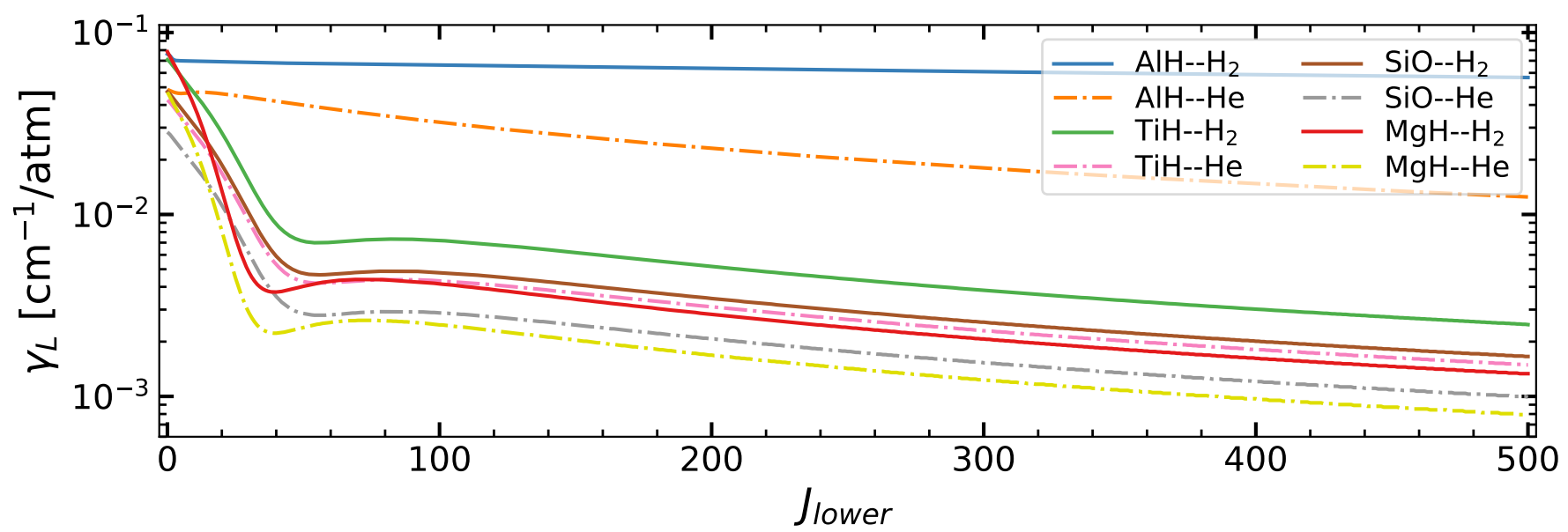

Figure 2. Example of used Lorentz coefficients for $\mathrm{AlH}$, TiH, $\mathrm{SiO}$, and $\mathrm{MgH}$. The AlH Lorentz coefficients are assumed to be equal to $\mathrm{CO}$, because they both have the same dipole moments. For other absorbers, the Lorentz coefficient are decreasing sharply up to $J=40$ similar to $\mathrm{HCl}$ because they all have high dipole moments. $4^{\text {th }}$ order Padé equation (Eq. 4 including the constrains introduced in (Eq. 5) is used and the coefficients are reported in Table 3 in order to reproduce these broadening data (see $§ 3.3$ for more details.). 

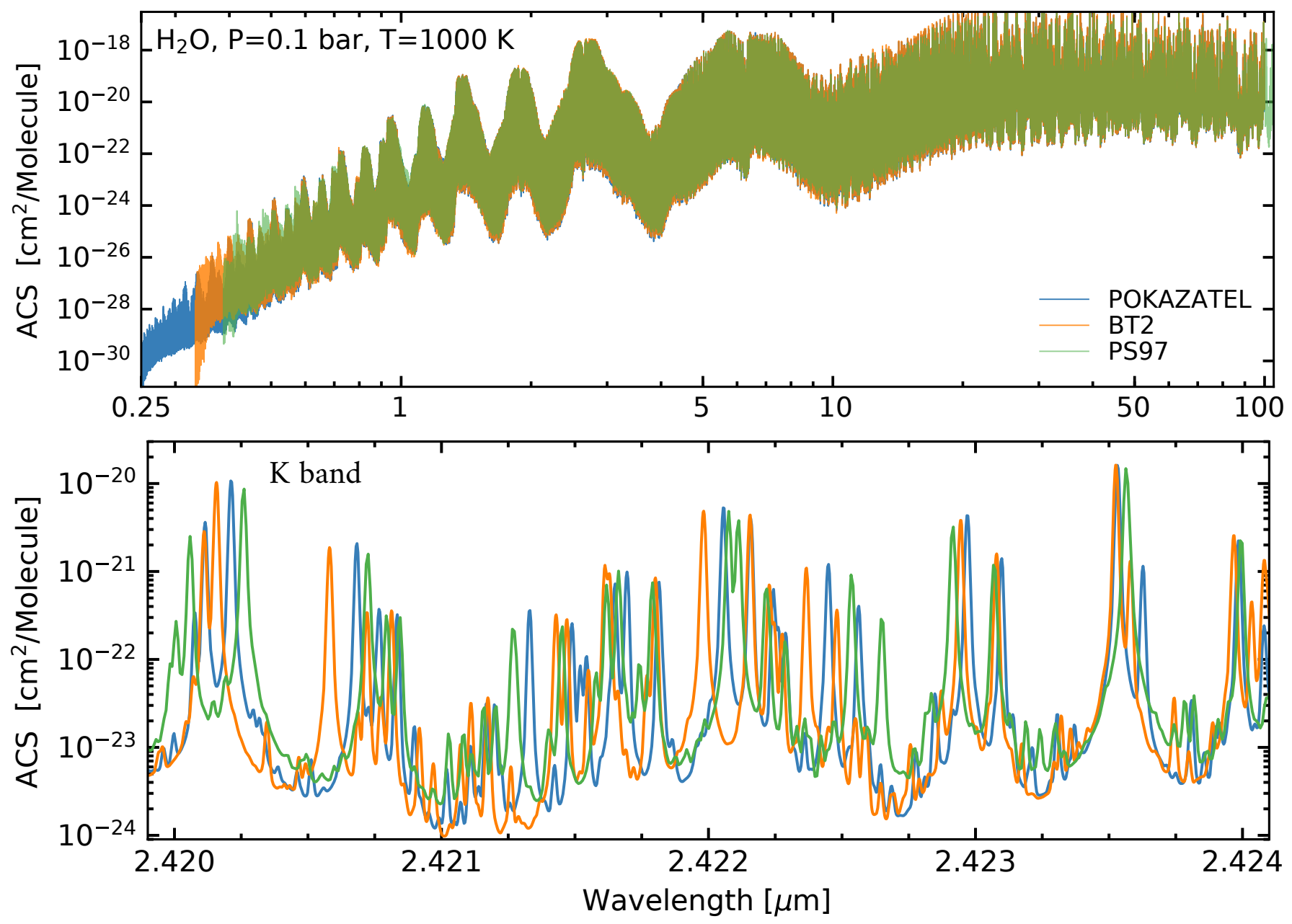

Figure 3. Comparison of different $\mathrm{H}_{2} \mathrm{O}$ ACS for 0.1 bar and $1000 \mathrm{~K}$, including POKAZATEL, BT2, and PS97. Note, that $P O K A Z A T E L$ and BT2 line lists are from ExoMol group, however, POKAZATEL is the most recent version. In addition, the Schwenke ACS is generated by Freedman et al. (2014) using Partridge \& Schwenke (1997) water line list (named PS97). Their expanded comparisons for $2.4 \mu \mathrm{m}$ (bottom) shows some differences in their line positions, intensities. Additionally, there are some extra lines from POKAZATEL that are not exist in others. Although they are weak, but they can change the opacity continuum, and so their inclusion is important in particularly windows spectral regions (see 7 for more discussion). These differences result from the implemented quantum mechanic level of complexity, and they could bias the interpretation of medium-to-high-resolution observed data. 


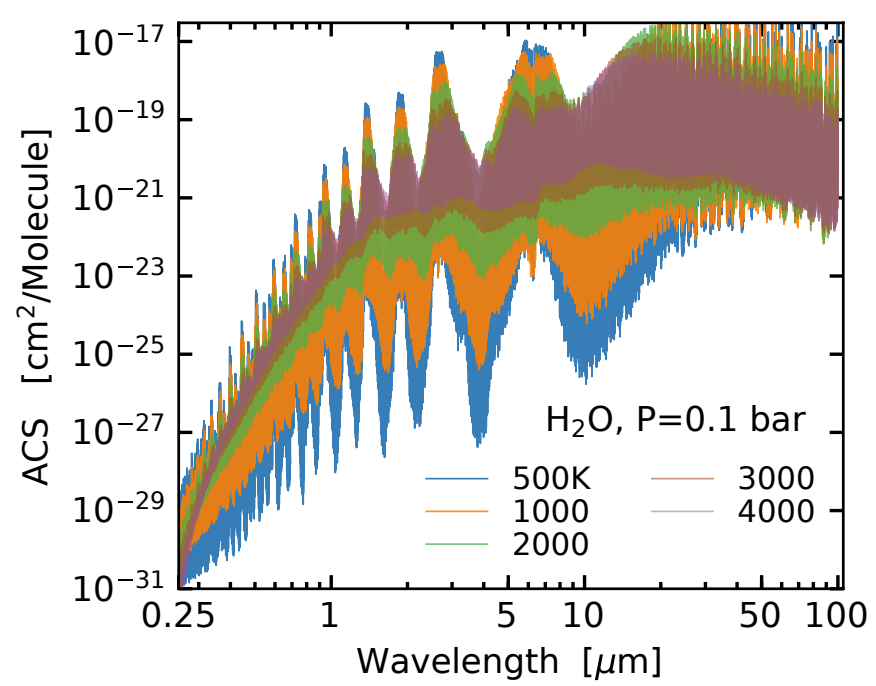

Figure 4. Example of $\mathrm{H}_{2} \mathrm{O} P O K A Z A T E L$ ACS spectra for 0.1 bar and multiple temperatures. For the same pressure, ACS with higher temperature have broader Doppler linewidth, and this will results in larger opacity in the line core comparing to the wings. 

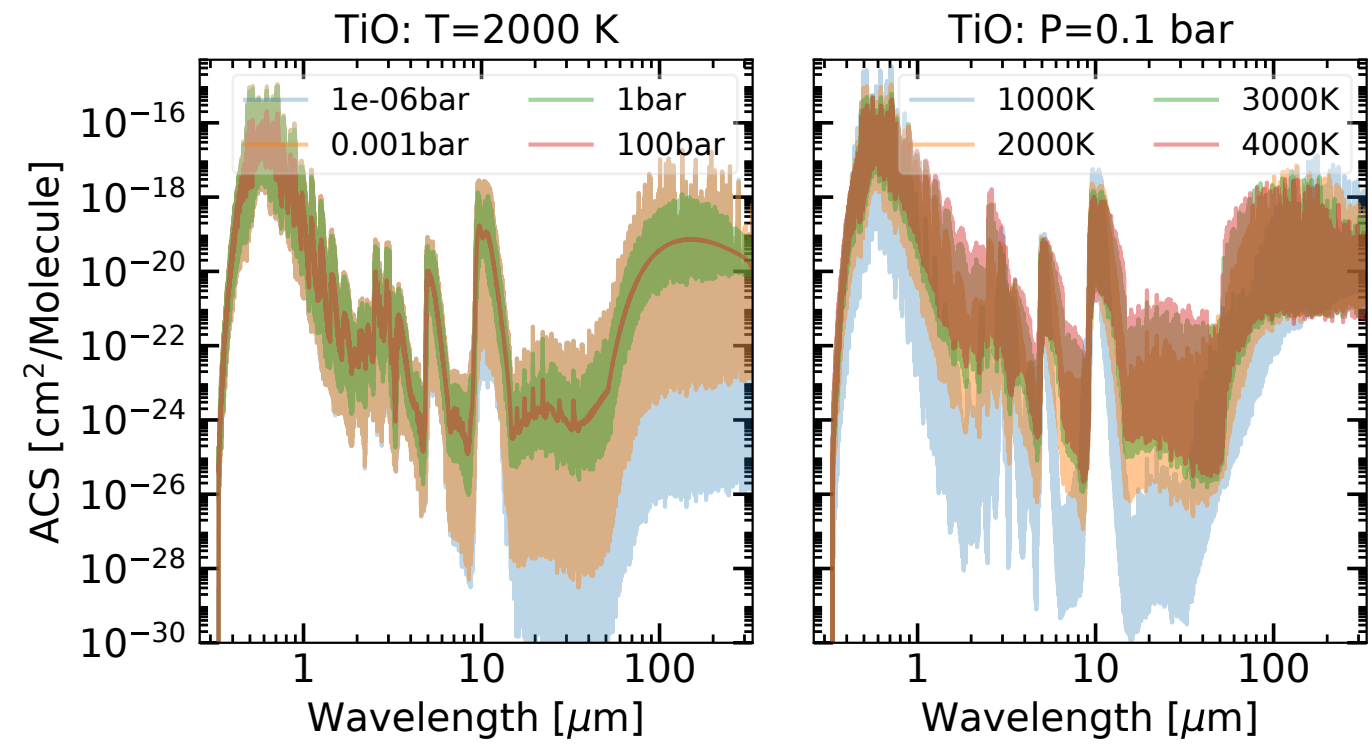

Figure 5. Example of the pre-generated TiO-TOTO ACS data: at a constant pressure, Doppler effect is the main source of line-width, particularly at low wavelengths (right). In contrast, at constant temperature, the higher pressure shows the most broadening as a result of collisional effect (left). Note that Lorentz width is dependent on the temperature as well. We used (McKemmish et al. 2019b) line list to generated these ACS data. 


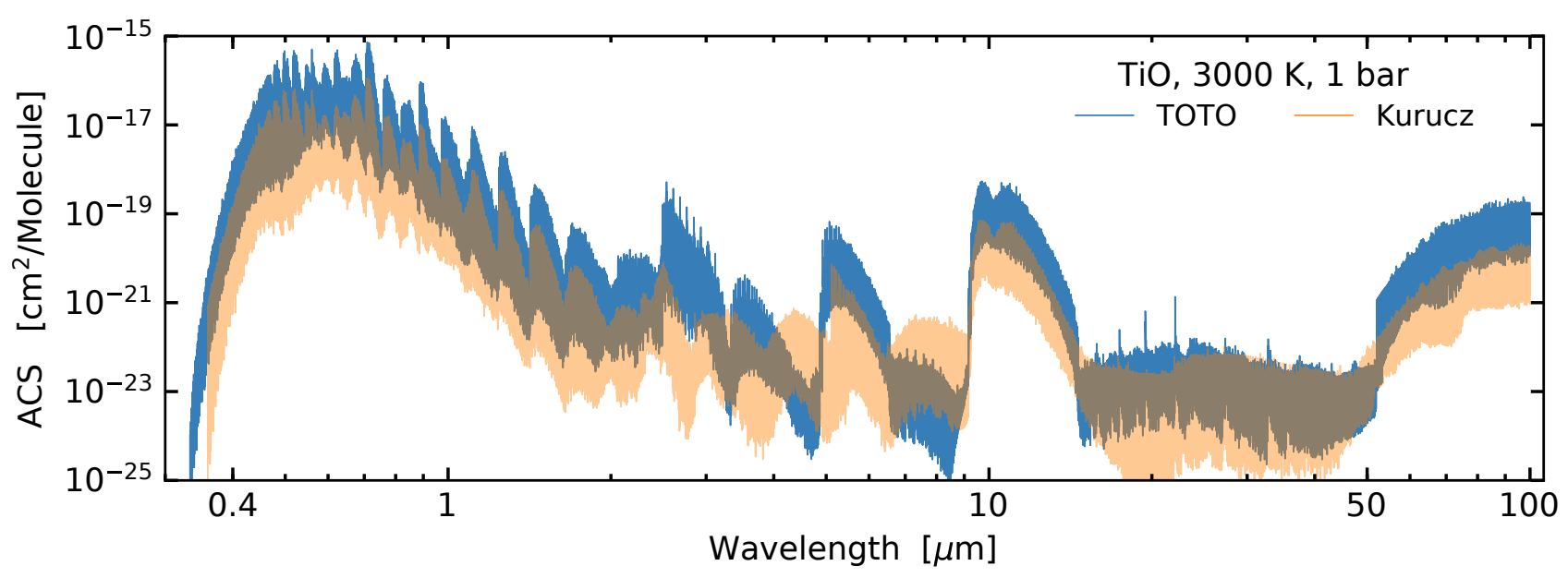

Figure 6. Compare $\mathrm{TiO}$ absorption cross-sections generated from TOTO and Kurucz line list for 1 bar and $3000 \mathrm{~K}$. Note that there are noticeable differences in the whole spectral range between both cases. $2.5-9 \mu m$ region is showing the highest inconsistency between two spectra. Overall, the TiO TOTO-ACS is showing a larger value than the Kurucz data, which might be due to its large number of transitions as well as the modified electronic states which results in shift in some spectral bands. 


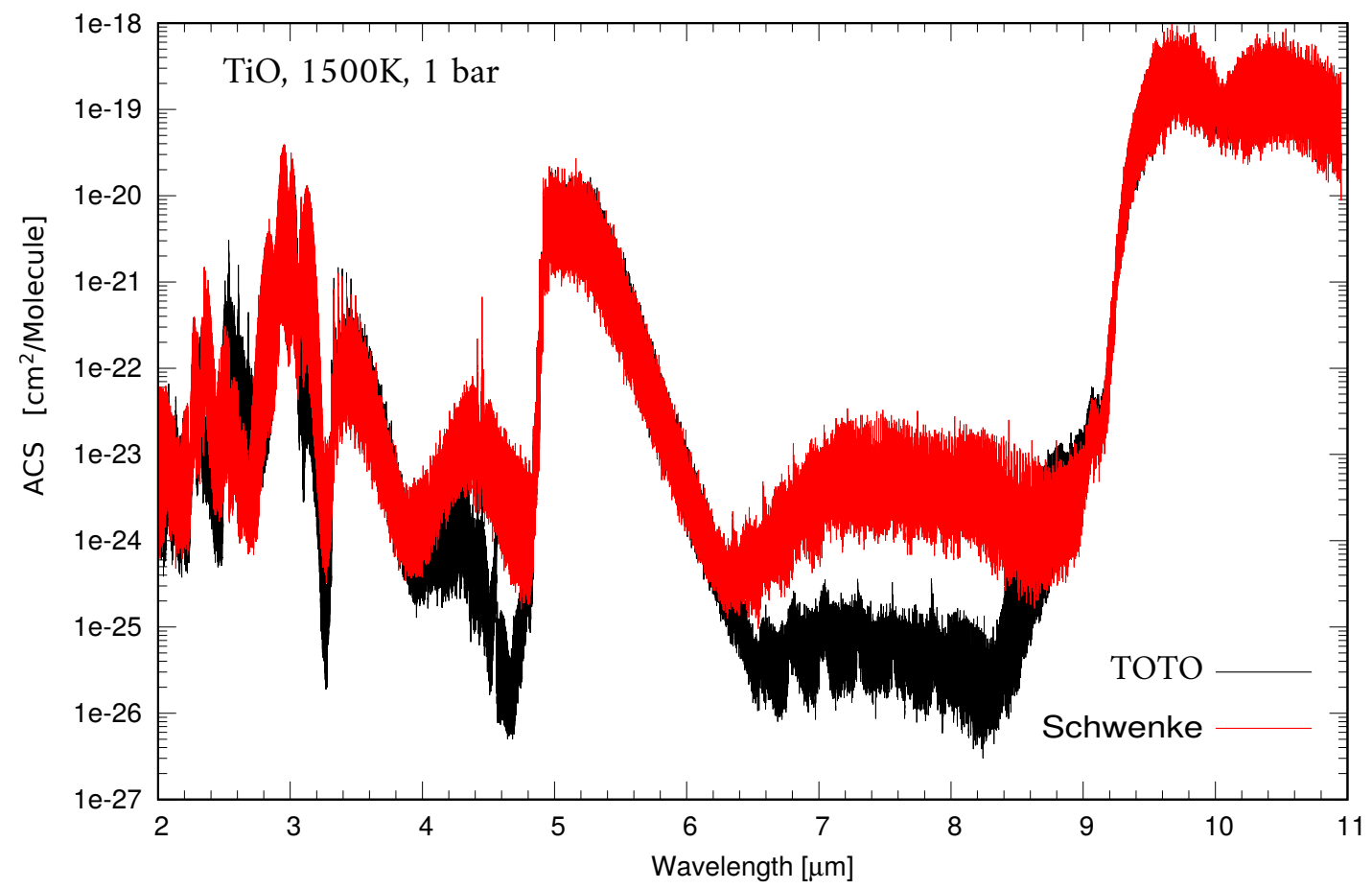

Figure 7. Compare TiO absorption cross-sections generated from the TOTO and Schwenke line list for 1 bar and $1500 \mathrm{~K}$. There are noticeable differences in the whole spectral range between both cases. The highest inconsistency is at spectral regions $\sim 4-5$ and $6.5-8.5 \mu \mathrm{m}$, that results in a dramatic bias in modeling low and high resolution synthetic emission or transmission spectra. One possible explanation would be the difference between the number of rotational levels that have been accounted by both TOTO and Schwenke linelist. 

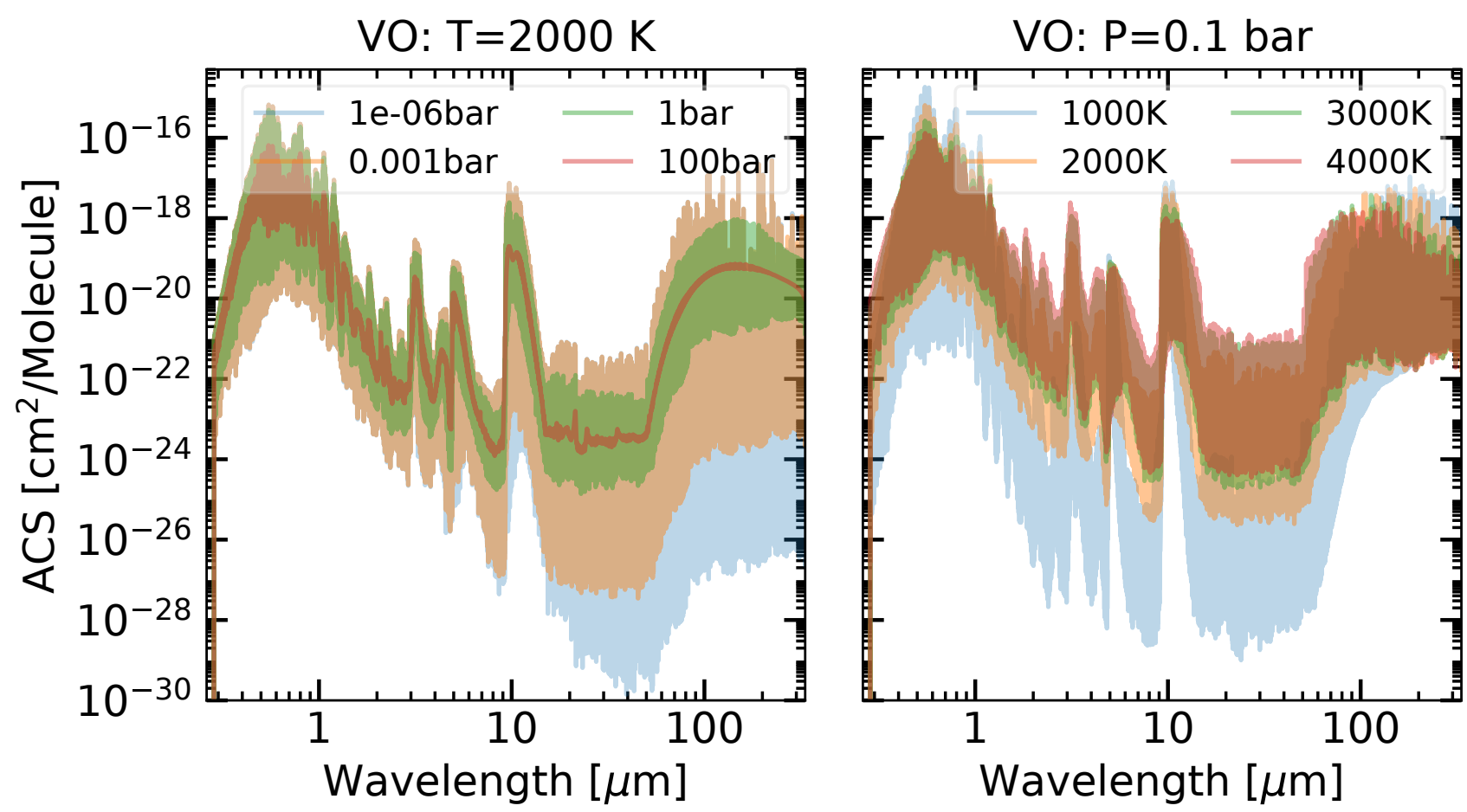

Figure 8. Example of our pre-generated VO-VOMYT absorption cross-sections: at a constant pressure, Doppler effect is the main reason of controlling the line-width, particularly at low wavelengths (right). In contrast, at constant temperature, the higher pressure shows the most broadening as a result of collisional effect (left). These pre-generated ACSs are from (McKemmish et al. 2016) line list data, which consists of the 13 low-lying electronic state and have $\sim 227$ million lines. 


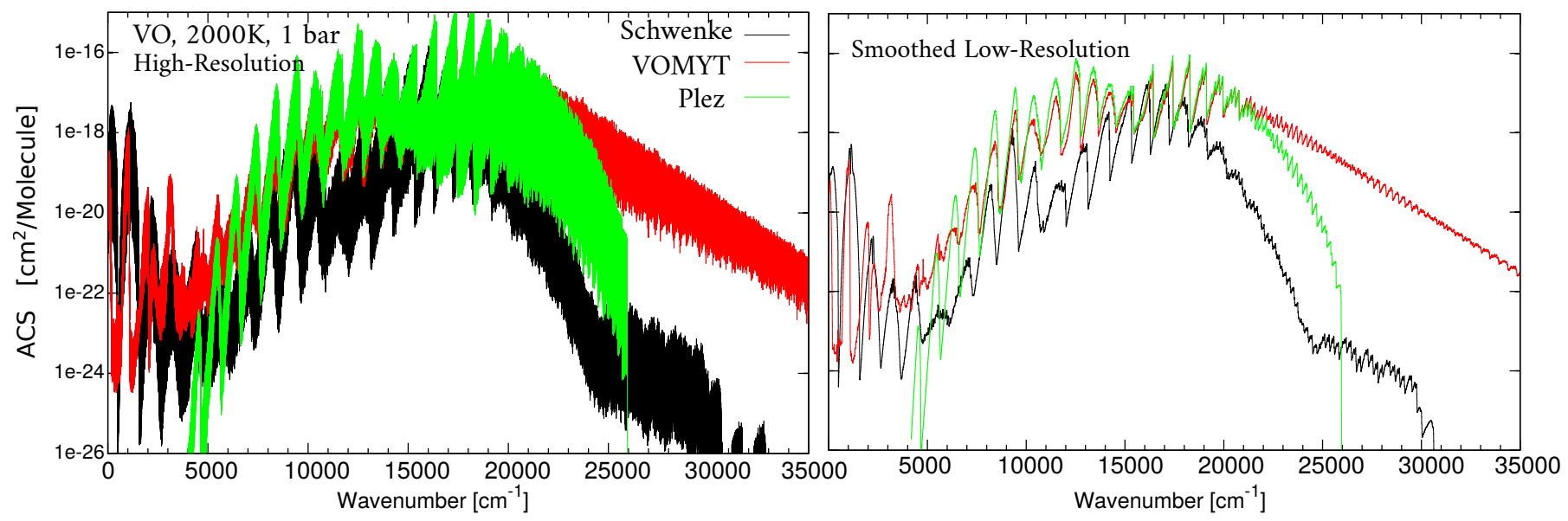

Figure 9. Comparison of different VO absorption cross-sections generated from different line lists: Schwenke, VOMYT, and Plez at $2000 \mathrm{~K}$ and 1 bar. VOMYT line list (McKemmish et al. 2016) includes the 13 low-lying states and $\sim 227$ million transitions, and hence the intensity of its spectra (red) is higher than other line lists (except Plez around $10000 \mathrm{~cm}^{-1}$ which resulted from A-X transitions). In contrast, Plez line list (Plez 1999)(green) has only the A-X, B-X, and C-X bands with no infrared transitions, and so it is only showing data at $5000-25000 \mathrm{~cm}^{-1}(0.4-2 \mu \mathrm{m})$. The central message of this figure is to show the difference between VO-ACS data from different line lists is remarkable, and naive implementation of VO ACS data could result in false conclusions and misinterpretation of the observational exoplanet/brown-dwarf spectra. 

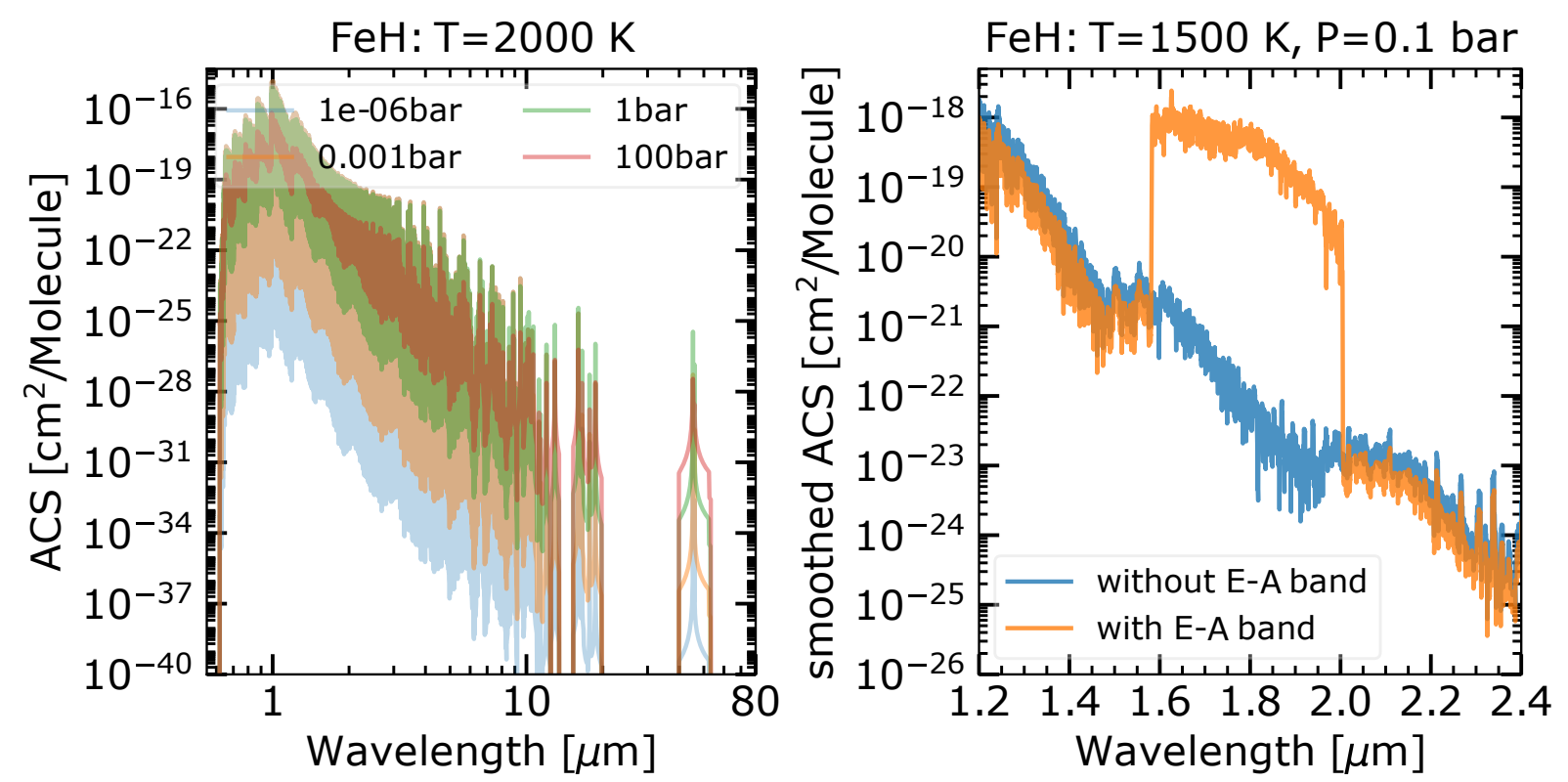

Figure 10. (left) An example of our FeH ACS spectra generated from Dulick et al. (2003) linelist for multiple pressures and 2000 K. (right) Comparison of the FeH absorption cross-sections generated with (orange) and without (blue) including the $E-A$ band. The discrepancies in these ACS data are due to the lack of sufficient accuracy in the $E-A$ band. 

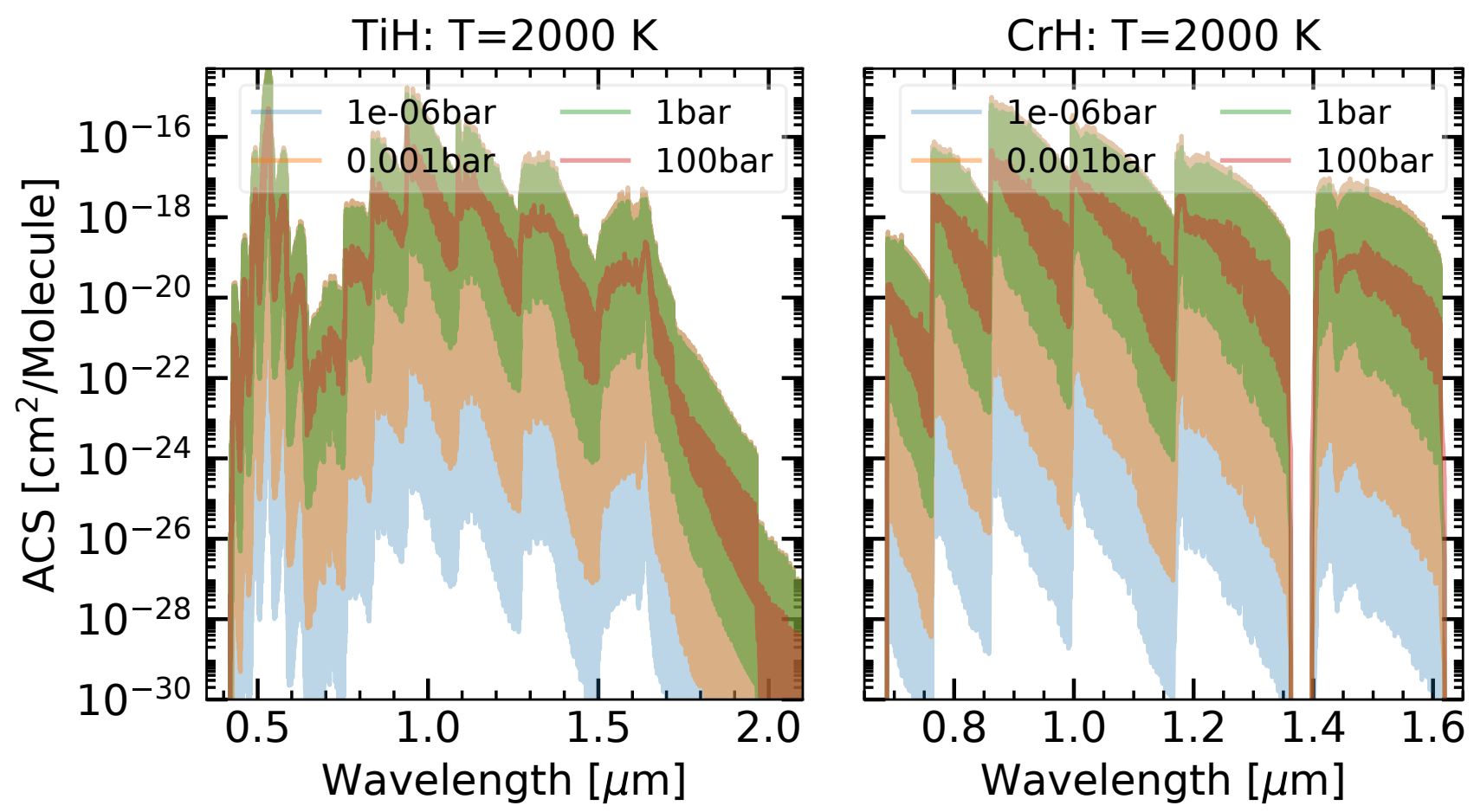

Figure 11. Example of our pre-generated $\mathrm{TiH}$ (left) and $\mathrm{CrH}$ (right) absorption cross-sections data at $2000 \mathrm{~K}$, and multiple pressures. The $\mathrm{TiH}$ line list have been generated using a combination of ab initio data as well as spectroscopic measurements for $0.4-2.1 \mu \mathrm{m}$ (A-X and B-X systems) by Burrows et al. (2005). The CrH line lists are also used from Burrows et al. (2002) for $0.65-1.65 \mu \mathrm{m}$ (A-X system). These linelists are limited to these wavelength range, and future laboratory/ab-initio studies are needed to extend these spectra to higher wavelengths (e.g., for $\mathrm{CrH}$ at $1.38 \mu \mathrm{m}$ gap). 

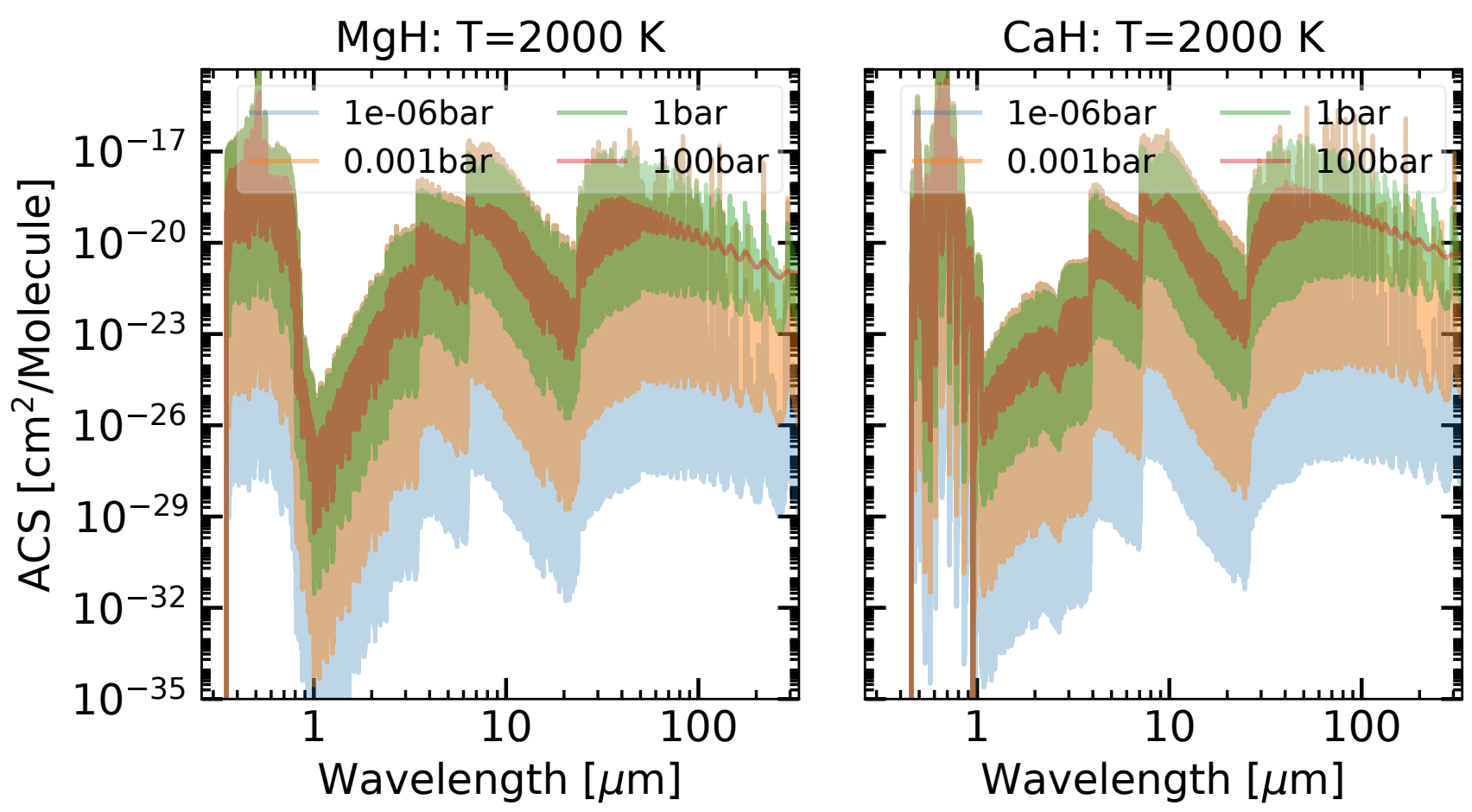

Figure 12. Example of our pre-generated $\mathrm{MgH}$ (left) and $\mathrm{CaH}$ (right) absorption cross-sections data at $2000 \mathrm{~K}$, and multiple pressures. The generated $\mathrm{MgH}$ is a combination of the laboratory line list from GharibNezhad et al. (2013) for $0.35-1.2 \mu \mathrm{m}(A-X$ and $B-X$ transitions) as well as theoretical infrared line lists from Yadin et al. (2012). The CaH includes laboratory measured line lists from Alavi \& Shayesteh (2017b); Li et al. (2012) for 0.45-1.0 ( $A-X$ and $B-X), 0.7-11$ (rovibrational transitions) by Shayesteh et al. (2004), and the $\mu \mathrm{m}(E-X)$ transitions $\sim 0.48 \mu \mathrm{m}$ by Li et al. (2012). CaH is also consists of theoretical line list generated by (Yadin et al. 2012) for rovibrational transitions. See Table 4 for further details. 

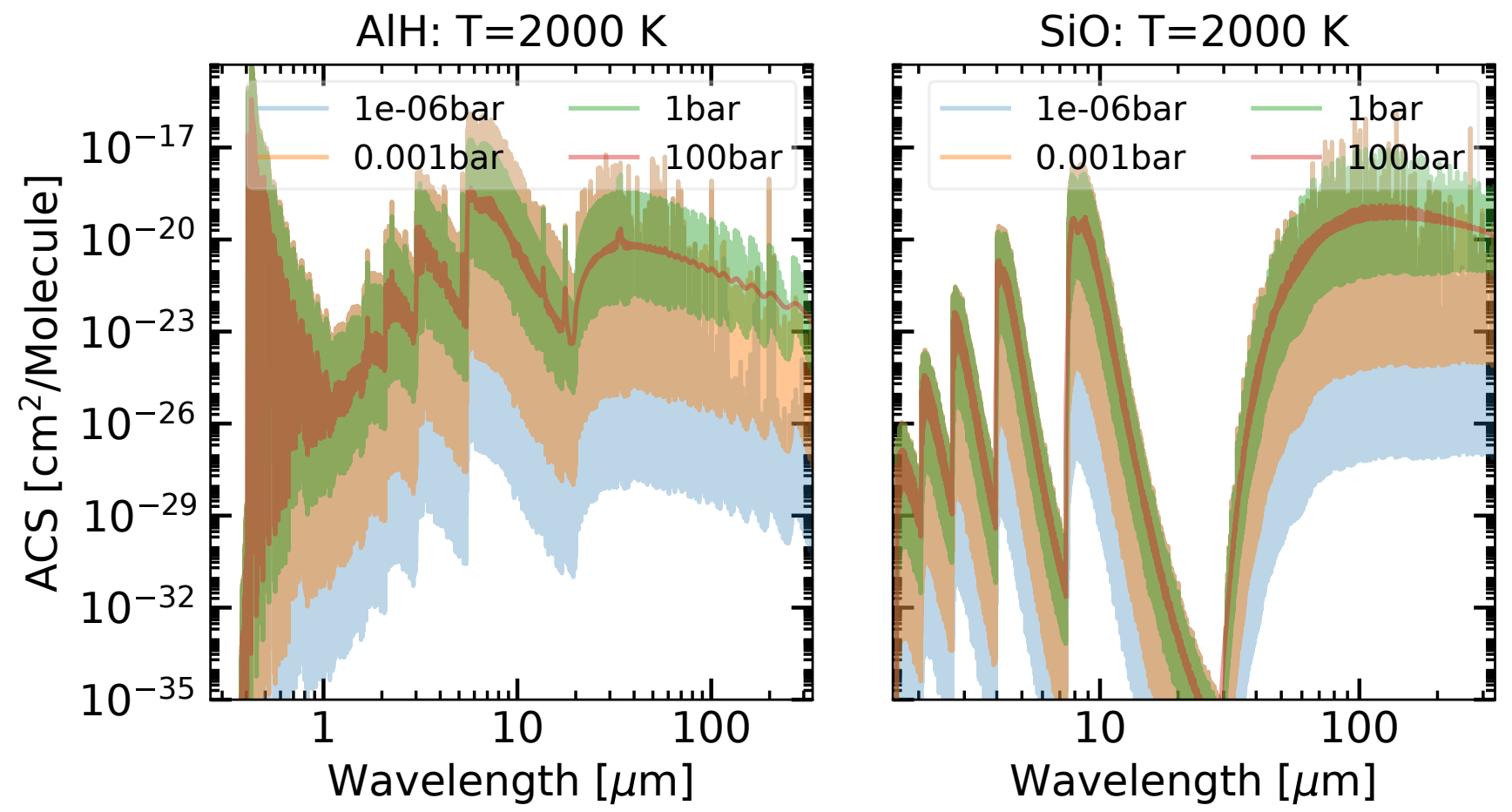

Figure 13. Example of our pre-generated $\mathrm{AlH}$ (left) and $\mathrm{SiO}$ (right) absorption cross-sections data at a $2000 \mathrm{~K}$ and multiple pressures. The AlH line list (named WYLLoT) is obtained from experimentally-calculated electronic states of $X$ and $A$ by Yurchenko et al. (2018) and so consists of A-X and X-X transitions. On the other hand, the SiO line list in our study has been computed using the available recorded $\mathrm{SiO}$ from both laboratory and sunspot spectra as well as the computed dipole moments by Barton et al. (2013).According to Barton et al. (2013), the SiO lines above $10000 \mathrm{~cm}^{-1}$ are very weak and so any lines between $10000-65000 \mathrm{~cm}^{-1}$ was filtered in their study. 


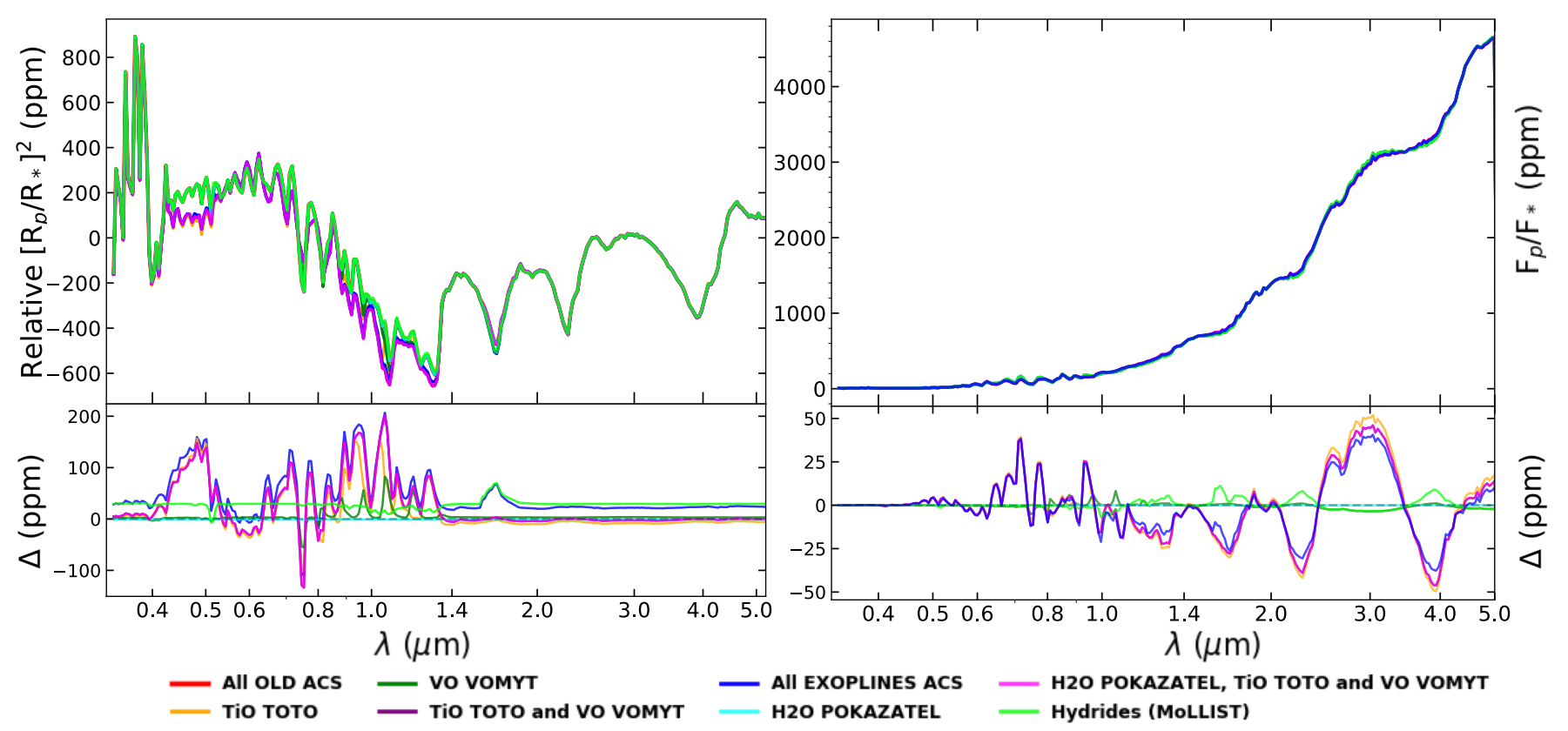

Figure 14. Impact of pre-generated opacities in the transmission and emission spectrum of a representative Ultra-Hot Jupiter. Transmission (Left) and Emission (Right) spectra calculated using opacities from all old ACSs (red) compared to all EXOPLINES ACSs (blue). Choice of linelists for TiO (TOTO vs Schwenke, in orange), VO (Plez vs VOMYT, in green), $\mathrm{H}_{2} \mathrm{O}(\mathrm{Schwenke}$ vs POKAZATEL, in cyan), and metal hydrides (in lime, see Table 7 references) along with combination differences of metal oxides ( $\mathrm{TiO}$ and $\mathrm{VO}$, in purple) as well as $\mathrm{H}_{2} \mathrm{O}$ (magenta) showing notable residuals relative to all old ACS data (red) of up to 300 ppm, primarily in the optical bandpass, between $0.3-1.0 \mu \mathrm{m}$ at $J W S T$ fiducial resolution at $\mathrm{R}=100$ for transmission (Bottom Left) and up to $80 \mathrm{ppm}$ in the infrared between 1.0-5.0 $\mu \mathrm{m}$ for thermal emission (Bottom Right), despite no significant shape differences amongst the spectra. In both cases, TiO produces the largest residual differences. Therefore, significant bias may arise with the choice of linelist in model atmospheres, especially when characterizing ultra hot Jupiter atmospheres with $J W S T$. 


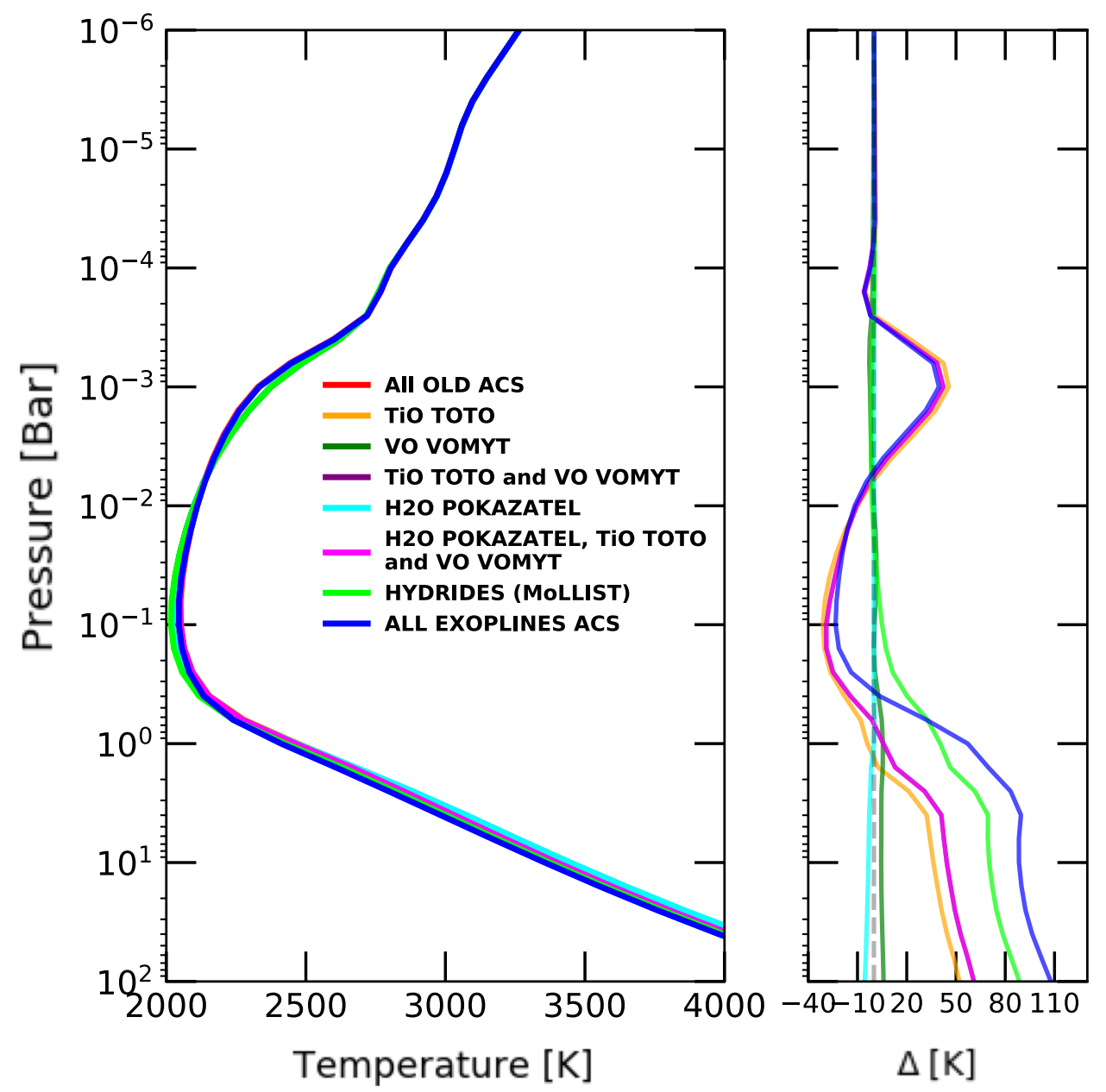

Figure 15. The impact of pre-generated opacities in the atmospheric structure of a representative Ultra-Hot Jupiter.(Left) Thermal structure calculated using pre-generated opacities from all old ACS (red) (Freedman et al. (2008), See Table 7), compared to EXOPLINES (blue). (Right) Independent effects of TiO (TOTO), VO (VOMYT), $\mathrm{H}_{2} \mathrm{O}$ (POKAZATEL), metal hydrides $(\mathrm{FeH} / \mathrm{MgH} / \mathrm{CaH} / \mathrm{CrH}-\mathrm{MoLLIST}$, and AlH-WYLLoT) (orange, green, cyan and lime respectively) as well as combination differences of all EXOPLINES ACS (blue) show variation in the thermal profile of up to $130 \mathrm{~K}$ for the same planet. The largest variations in the thermal profile are purely due to ACS differences of metal hydrides and oxides that are dominant in highly irradiated planet atmospheres. 

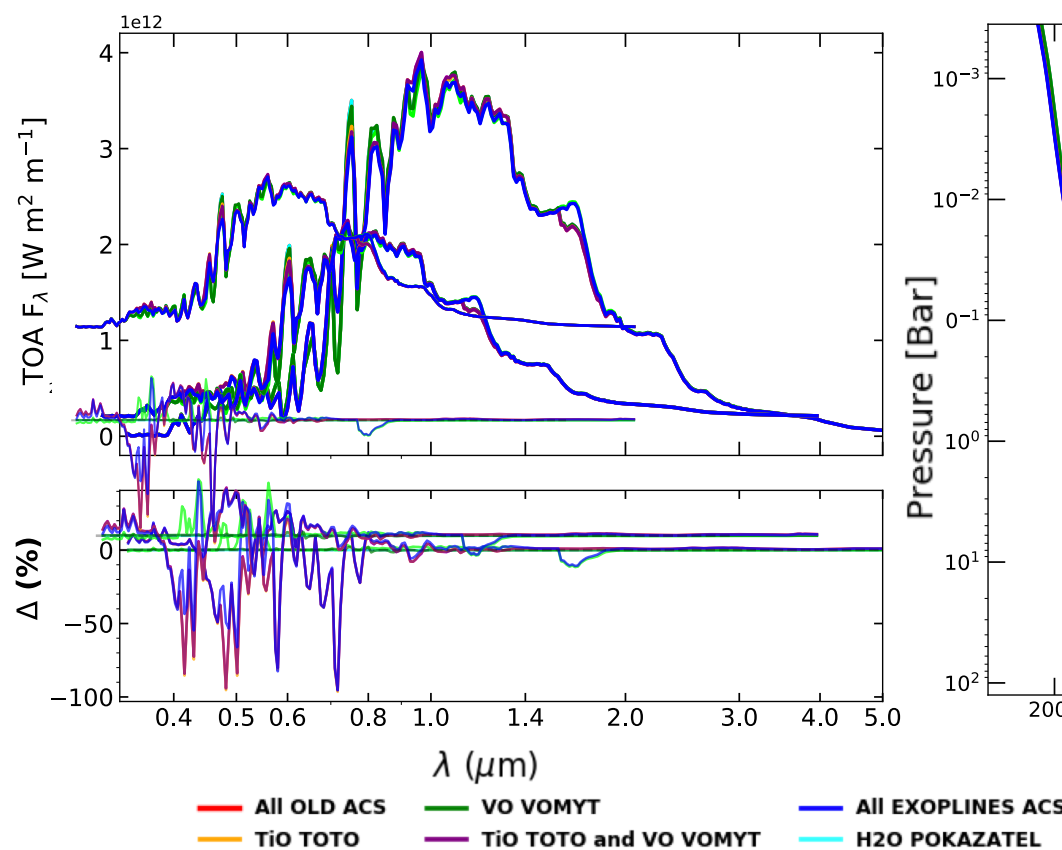
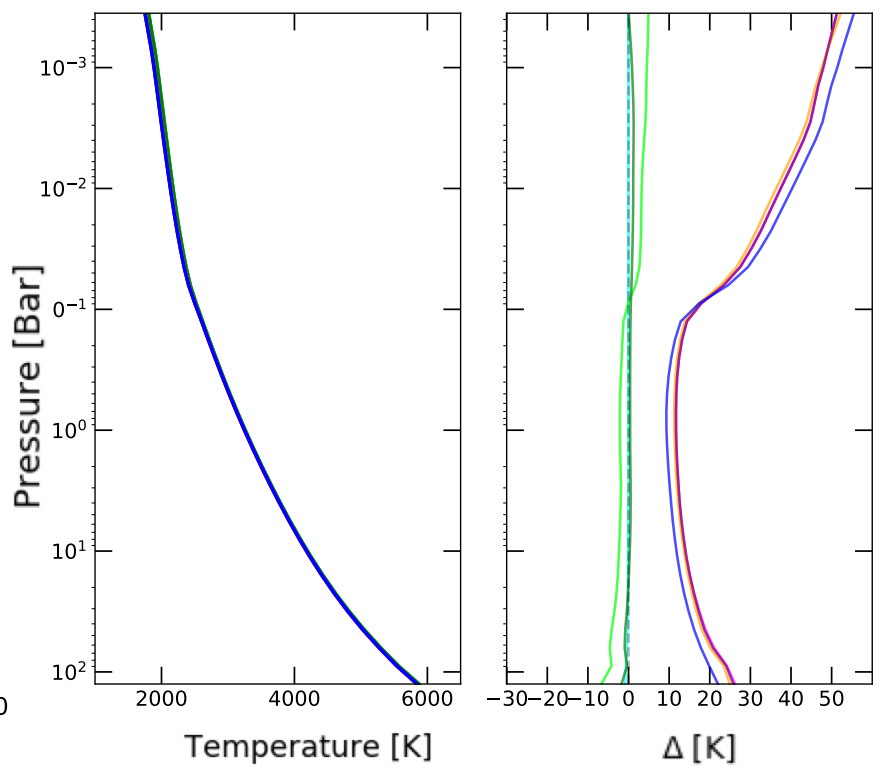

H2O POKAZATEL, TIO TOTO and VO VOMYT Hydrides (MoLLIST)

Figure 16. Impact of pre-generated opacities in characterizing a fiducial M-dwarf atmosphere, with $\mathrm{T}_{\text {eff }}$ of $3000 \mathrm{~K}$, log (g) of $5 \mathrm{~cm} . \mathrm{s}^{-2}$, solar M/H, and C/O of 0.5. (Left) Model spectrum of the fiducial M-dwarf (showing top-of-atmosphere (TOA) flux) varying opacities calculated from all old ACS (red) compared to all EXOPLINES ACS (blue). Spectrum ( $\mathrm{R}=100$, covering $0.3-5 \mu \mathrm{m})$ calculated with TiO TOTO linelist (orange) attributes to largest residual differences in the spectral shape, with up to $125 \%$ in the optical bandpass below $1 \mu \mathrm{m}$, followed by $30 \%$ in residual differences caused purely due to the effect of metal hydride opacities (MoLLIST, lime) below $1 \mu \mathrm{m}$, and up to $12 \%$ between 1.4-2.0 $\mu \mathrm{m} . \mathrm{H}_{2} \mathrm{O}$ (POKAZTEL, cyan) and VO (VOMYT, green) alone do not induce noteworthy differences within the spectra. (Right) Atmospheric structure of fiducial M-dwarf, with respective residuals in the thermal profile. Consistent with the spectral differences, TiO linelist contributes to significant deviations within the T-P profile with up to $50 \mathrm{~K}$ above 0.1 bar in pressure, and up to $20 \mathrm{~K}$ down to 100 bars. The effect of both metal oxides ( $\mathrm{TiO}$ and $\mathrm{VO}$, purple), combined with $\mathrm{H}_{2} \mathrm{O}$ (magenta) and along with all new opacities (EXOPLINES, blue) cause deviations in atmospheric structure of up to a maximum of $60 \mathrm{~K}$. 


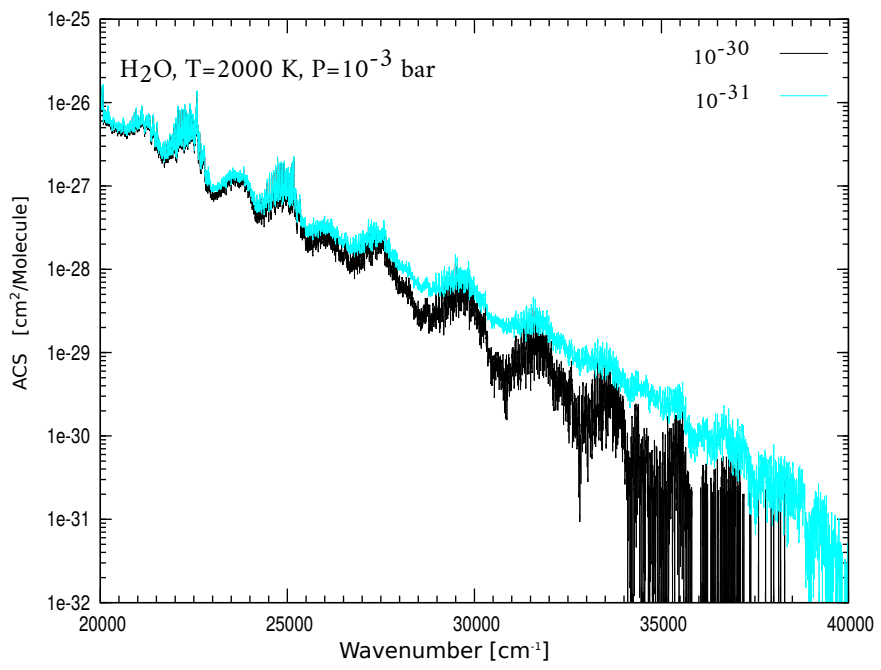

Figure 17. The impact of intensity cut-off on generating the $\mathrm{H}_{2} \mathrm{O} P O K A Z A T E L$ ACS data. Following Maxwell-Boltzmann distribution, the population of energy levels with high $J$ quantum number increases with temperature. As a result, weak lines become stronger at high temperatures, and so insufficient intensity cut-off value results in accurate ACS. For instance, the choice of $10^{-30}$ (black) for intensity cut-off eliminate more lines in generating ACS data comparing to $10^{-31} \mathrm{~cm}^{-1} /\left(\mathrm{molecule} \mathrm{cm}^{-2}\right)$ (cyan). The reported cut-off values refer to $296 \mathrm{~K}$. 


\section{REFERENCES}

Akpinar, S., \& Akbalik, F. 2010, Journal of Molecular Structure: THEOCHEM, 942, 98 , doi: https://doi.org/10.1016/j.theochem.2009.12.002

Alavi, S. F., \& Shayesteh, A. 2017a, MNRAS, 474, 2, doi: 10.1093/mnras/stx2681

—. 2017b, MNRAS, 474, 2, doi: 10.1093/mnras/stx2681

Alberti, M., Weber, R., Mancini, M., Fateev, A., \& Clausen, S. 2015, JQSRT, 157, 14 , doi: https://doi.org/10.1016/j.jqsrt.2015.01.016

Allard, F., Hauschildt, P. H., \& Schwenke, D. 2000, ApJ, 540, 1005, doi: 10.1086/309366

Allard, N. F., Spiegelman, F., \& Kielkopf, J. F. 2016, A\&A, 589, A21, doi: 10.1051/0004-6361/201628270

Allard, N. F., Spiegelman, F., Leininger, T., \& Molliere, P. 2019, A\&A, 628, A120, doi: 10.1051/0004-6361/201935593

Amiot, C., Azaroual, E. M., Luc, P., \& Vetter, R. 1995, JChPh, 102, 4375, doi: 10.1063/1.469486

Amundsen, D. S., Mayne, N. J., Baraffe, I., et al. 2016, A \& A, 595, A36

Anderson, P. W. 1949, Physical Review, 76, 647

Andersson, N., Balfour, W. J., Bernath, P. F., Lindgren, B., \& Ram, R. S. 2003, JChPh, 118, 3543, doi: 10.1063/1.1539848

Arcangeli, J., Désert, J.-M., Line, M. R., et al. 2018, ApJ, 855, L30

Babrov, H., Ameer, G., \& Benesch, W. 1960, The Journal of Chemical Physics, 33, 145

Balfour, W. J., Brown, J. M., \& Wallace, L. 2004, JChPh, 121, 7735, doi: 10.1063/1.1797731

Balfour, W. J., \& Cartwright, H. M. 1975a, Canadian Journal of Physics, 53, 1477, doi: 10.1139/p75-188

—. 1975b, Chemical Physics Letters, 32, 82, doi: 10.1016/0009-2614(75)85173-6

Balfour, W. J., Merer, A. J., Niki, H., Simard, B., \& Hackett, P. A. 1993, JChPh, 99, 3288, doi: 10.1063/1.465138

Baranger, M. 1958, Physical Review, 111, 481, doi: 10.1103/PhysRev.111.481

Barber, R. J., Tennyson, J., Harris, G. J., \& Tolchenov, R. N. 2006, MNRAS, 368, 1087. https://arxiv.org/abs/0601236

Barklem, P. S., \& Collet, R. 2016, A\&A, 588, A96, doi: 10.1051/0004-6361/201526961

Barrow, R., \& Stone, T. 1975, Journal of Physics B: Atomic and Molecular Physics, 8, L13

Barton, E. J., Hill, C., Yurchenko, S. N., et al. 2017, JQSRT, 187, 453. https://arxiv.org/abs/1610.09008
Barton, E. J., Yurchenko, S. N., \& Tennyson, J. 2013, MNRAS, 434, 1469, doi: 10.1093/mnras/stt1105

Batalha, N., \& Rooney, C. 2020, doi: 10.5281/zenodo.4206648

Batalha, N. E., Marley, M. S., Lewis, N. K., \& Fortney, J. J. 2019, ApJ, 878, 70, doi: 10.3847/1538-4357/ab1b51

Batalha, N. M. 2014, Proceedings of the National Academy of Sciences, 111, 12647, doi: 10.1073/pnas.1304196111

Bauschlicher, C. W., Ram, R. S., Bernath, P. F., Parsons, C. G., \& Galehouse, D. 2001, JChPh, 115, 1312, doi: $10.1063 / 1.1377892$

Bean, J. L., Stevenson, K. B., Batalha, N. M., et al. 2018, PASP, 130, 114402, doi: 10.1088/1538-3873/aadbf3

Bernath, P. 2005, Spectra of Atoms and Molecules, 2nd edn. (Oxford University Press)

—. 2020a, ApJ, 895, 87, doi: 10.3847/1538-4357/ab7cd0

Bernath, P. F. 2020b, JQSRT, 240, 106687, doi: https://doi.org/10.1016/j.jqsrt.2019.106687

Bézard, B., Fedorova, A., Bertaux, J.-L., Rodin, A., \& Korablev, O. 2011, Icarus, 216, 173

Birkby, J. L., de Kok, R. J., Brogi, M., et al. 2013, MNRAS, 436, L35, doi: 10.1093/mnrasl/slt107

Bittner, D. M., \& Bernath, P. F. 2018, ApJS, 236, 46, doi: 10.3847/1538-4365/aabfe8

Brandes, G. R., \& Galehouse, D. C. 1985, JMS, 109, 345, doi: 10.1016/0022-2852(85)90317-0

Brogi, M., \& Line, M. R. 2019, AJ, 157, 114

Buldyreva, J., Lavrentieva, N., \& Starikov, V. 2011, Collisional Line Broadening and Shifting of Atmospheric Gases (World Scientific Publishing Co)

Burrows, A., Dulick, M., Bauschlicher, C. W., J., et al. 2005, ApJ, 624, 988, doi: 10.1086/429366

Burrows, A., Dulick, M., C. W. Bauschlicher, J., et al. 2005, ApJ, 624, 988, doi: 10.1086/429366

Burrows, A., Ram, R. S., Bernath, P., Sharp, C. M., \& Milsom, J. A. 2002, ApJ, 577, 986, doi: 10.1086/342242

Burrows, A., \& Sharp, C. 1999, ApJ, 512, 843

Campbell, J. M., Klapstein, D., Dulick, M., Bernath, P. F., \& Wallace, L. 1995, ApJS, 101, 237, doi: 10.1086/192238

Cappelletti, D., Bartolomei, M., Sabido, M., et al. 2005, The Journal of Physical Chemistry A, 109, 8471

Cheung, A.-C., Taylor, A., \& Merer, A. 1982, JMS, 92, 391 , doi: https://doi.org/10.1016/0022-2852(82)90110-2

Chubb, K. L., Rocchetto, M., Yurchenko, S. N., et al. 2020, arXiv e-prints, arXiv:2009.00687. https://arxiv.org/abs/2009.00687

Conway, E. K., Gordon, I. E., Tennyson, J., et al. 2020, Atmospheric Chemistry and Physics, 20, 10015, doi: 10.5194/acp-20-10015-2020 
Cushing, M. C., Rayner, J. T., Davis, S. P., \& Vacca, W. D. 2003, ApJ, 582, 1066, doi: 10.1086/344525

Cushing, M. C., Rayner, J. T., \& Vacca, W. D. 2005, ApJ, 623, 1115, doi: 10.1086/428040

Dayou, F., \& Balança, C. 2006, A\&A, 459, 297, doi: 10.1051/0004-6361:20065718

Delfosse, X., Tinney, C. G., Forveille, T., et al. 1997, A\&A, 327, L25

Devi, M. V., Chris Benner, D., Smith, M., et al. 2012, Journal of Quantitative Spectroscopy and Radiative Transfer, 113, 1013 , doi: https://doi.org/10.1016/j.jqsst.2012.02.010

Devi, M. V., Predoi-Cross, A., Chris Benner, D., et al. 2004, Journal of Molecular Spectroscopy, 228, 580 , doi: https://doi.org/10.1016/j.jms.2004.05.006

Dulick, M., C. W. Bauschlicher, J., Burrows, A., et al. 2003, ApJ, 594, 651, doi: 10.1086/376791

Fortney, J. J., Lodders, K., Marley, M. S., \& Freedman, R. S. 2008, ApJ, 678, 1419, doi: 10.1086/528370

Fortney, J. J., Mordasini, C., Nettelmann, N., et al. 2013, ApJ, 775, 80. https://arxiv.org/abs/1306.4329

Fortney, J. J., Robinson, T. D., Domagal-Goldman, S., et al. 2019, arXiv e-prints.

https://arxiv.org/abs/1905.07064

Freedman, R. S., Lustig-Yaeger, J., Fortney, J. J., et al. 2014, ApJS, 214. https://arxiv.org/abs/1409.0026

Freedman, R. S., Marley, M. S., \& Lodders, K. 2008, ApJS, 174, 504. https://arxiv.org/abs/0706.2374

Gamache, R. R., \& Vispoel, B. 2018, Journal of Quantitative Spectroscopy and Radiative Transfer, 217, 440

Gandhi, S., \& Madhusudhan, N. 2019, MNRAS, 485, 5817, doi: 10.1093/mnras/stz751

Gandhi, S., Brogi, M., Yurchenko, S. N., et al. 2020, MNRAS, doi: 10.1093/mnras/staa981

Gharib-Nezhad, E., Heays, A. N., Bechtel, H. A., \& Lyons, J. R. 2019, JQSRT, 239, 106649, doi: https://doi.org/10.1016/j.jqsrt.2019.106649

Gharib-Nezhad, E., \& Line, M. R. 2019, ApJ, 872, 27

GharibNezhad, E., Shayesteh, A., \& Bernath, P. F. 2013, MNRAS, 432, 2043, doi: 10.1093/mnras/stt510

Gierszal, S., Galica, J., \& Miś-Kuźmińska, E. 1998, The Journal of Chemical Physics, 108, 8976, doi: 10.1063/1.476343

Gordon, I. E., Rothman, L. S., Hill, C., et al. 2017, JQSRT, 203, 3, doi: 10.1016/j.jqsrt.2017.06.038

Goyal, J. M., Mayne, N., Sing, D. K., et al. 2018, MNRAS, 474, 5158, doi: 10.1093/mnras/stx3015

Greene, T. P., Line, M. R., Montero, C., et al. 2016, ApJ, 817, 17, doi: 10.3847/0004-637X/817/1/17
Grigoriev, I., Doucen], R. L., Boissoles, J., et al. 1999, JMS, 198, 249 , doi: https://doi.org/10.1006/jmsp.1999.7903

Grimm, S. L., Malik, M., Kitzmann, D., et al. 2021, ApJS, 253, 30, doi: 10.3847/1538-4365/abd773

Hargreaves, R. J., Hinkle, K. H., Bauschlicher, C. W., et al. 2010, AJ, 140, 919, doi: 10.1088/0004-6256/140/4/919

Hartmann, J. M., Boulet, C., Brodbeck, C., et al. 2002, JQSRT, 72, 117, doi: 10.1016/S0022-4073(01)00058-9

Hartmann, J.-M., Boulet, C., \& Robert, D. 2021, Collisional effects on molecular spectra: laboratory experiments and models, consequences for applications (Elsevier)

Hartmann, J.-M., Tran, H., Armante, R., et al. 2018, JQSRT, 213, 178, doi: 10.1016/j.jqsrt.2018.03.016

Herman, M. K., de Mooij, E. J. W., Jayawardhana, R., \& Brogi, M. 2020, arXiv e-prints, arXiv:2006.10743. https://arxiv.org/abs/2006.10743

Hodges, J. N., \& Bernath, P. F. 2018, ApJ, 863, 36, doi: $10.3847 / 1538-4357 /$ aacf07

Hoeijmakers, H. J., de Kok, R. J., Snellen, I. A. G., et al. 2015, A\&A, 575, A20, doi: 10.1051/0004-6361/201424794

Hopkins, W. S., Hamilton, S. M., \& Mackenzie, S. R. 2009, The Journal of Chemical Physics, 130, 144308, doi: 10.1063/1.3104844

Hörst, S. M., He, C., Lewis, N. K., et al. 2018, Nature Astronomy, 2, 303, doi: 10.1038/s41550-018-0397-0

Huang, G., Merer, A. J., \& Clouthier, D. J. 1992, JMS, 153, 32 , doi: https://doi.org/10.1016/0022-2852(92)90455-W

Hubeny, I., Burrows, A., \& Sudarsky, D. 2003, ApJ, 594, 1011, doi: $10.1086 / 377080$

Hübner, O., Hornung, J., \& Himmel, H.-J. 2015, JChPh, 143, 024309, doi: 10.1063/1.4926393

Humlíček, J. 1979, JQSRT, 21, 309

Iyer, A. R., Line, M. R., \& Gharib-Nezhad, E. In Prep

Kataria, T., Sing, D. K., Lewis, N. K., et al. 2016, ApJ, 821,9

Kempton, E. M.-R., Lupu, R., Owusu-Asare, A., Slough, P., \& Cale, B. 2017, PASP, 129, 044402, doi: 10.1088/1538-3873/aa61ef

Kempton, E. M. R., Bean, J. L., Louie, D. R., et al. 2018, PASP, 130, 114401, doi: 10.1088/1538-3873/aadf6f

Kirkpatrick, J. D. 2005, ARA\&A, 43, 195

Kirkpatrick, J. D., Barman, T. S., Burgasser, A. J., et al. 2006, ApJ, 639, 1120, doi: 10.1086/499622

Kirkpatrick, J. D., Henry, T. J., \& McCarthy, Donald W., J. 1991, ApJS, 77, 417, doi: 10.1086/191611

Kirkpatrick, J. D., Reid, I. N., Liebert, J., et al. 1999, ApJ, 519, 802, doi: 10.1086/307414

—. 2000, AJ, 120, 447, doi: 10.1086/301427

Kochanov, R. V., Gordon, I. E., Rothman, L. S., et al. 2016, JQSRT, 177, 15, doi: 10.1016/j.jqsrt.2016.03.005 
Kreidberg, L., Bean, J. L., Désert, J. M., et al. 2014, ApJL, 793, 2. https://arxiv.org/abs/1410.2255

Kurucz, R. L. 1992, RMxAA, 23, 45

Langhoff, S. R. 1997, ApJ, 481, 1007, doi: 10.1086/304077

Langhoff, S. R., \& Arnold, J. 1979, The Journal of Chemical Physics, 70, 852

Langhoff, S. R., \& Bauschlicher, C. W. 1993, Chemical Physics Letters, 211, 305 , doi: https://doi.org/10.1016/0009-2614(93)87064-A

Launila, O., \& Lindgren, B. 1996, JChPh, 104, 6418, doi: $10.1063 / 1.471362$

Li, G., Asfin, R. E., Domanskaya, A. V., \& Ebert, V. 2018, Molecular Physics, 116, 3495

Li, G., Harrison, J. J., Ram, R. S., Western, C. M., \& Bernath, P. F. 2012, JQSRT, 113, 67, doi: 10.1016/j.jqsrt.2011.09.010

Li, G., Harrison, J. J., Ram, R. S., Western, C. M., \& Bernath, P. F. 2012, JQSRT, 113, 67 , doi: https://doi.org/10.1016/j.jqsrt.2011.09.010

Line, M. R., Knutson, H., Wolf, A. S., \& Yung, Y. L. 2014, ApJ, 783, 70

Line, M. R., Stevenson, K. B., Bean, J., et al. 2016, AJ, 152, 203

Linton, C., Steimle, T. C., \& Frey, S. E. 2012, JMS, 279, 1, doi: 10.1016/j.jms.2012.07.017

Lockwood, G. W. 1972, ApJS, 24, 375, doi: 10.1086/190259

Lodders, K. 1999, ApJ, 519, 793, doi: 10.1086/307387

Lodders, K., \& Fegley, B., J. 2006, Chemistry of Low Mass Substellar Objects, ed. J. W. Mason, 1, doi: 10.1007/3-540-30313-8_1

Louie, D. R., Deming, D., Albert, L., et al. 2018, PASP, 130, 044401. https://arxiv.org/abs/1711.02098

Lupu, R. E., Zahnle, K., Marley, M. S., et al. 2014, ApJ, 784, 27, doi: 10.1088/0004-637X/784/1/27

Ma, Q., Tipping, R., \& Boulet, C. 2007, JQSRT, 103, 588

MacDonald, R. J. 2019, PhD thesis, University of Cambridge

Madhusudhan, N. 2019, ARA\&A, 57, 617, doi: 10.1146/annurev-astro-081817-051846

Malik, M., Kitzmann, D., Mendonça, J. M., et al. 2019, AJ, 157, 170, doi: 10.3847/1538-3881/ab1084

Mantz, A., Devi, V. M., Benner, D. C., et al. 2005, Journal of molecular structure, 742, 99

Marley, M. S., \& Robinson, T. D. 2015, ARA\&A, 53, 279. https://arxiv.org/abs/1410.6512

Matos, J. M. O., Malmqvist, P.-Å., \& Roos, B. O. 1987, JChPh, 86, 5032, doi: 10.1063/1.452679

McCormack, P., \& Oconnor, S. 1976, A\&AS, 26, 373

McKemmish, L. K., Masseron, T., Hoeijmakers, H. J., et al. 2019a, MNRAS, 488, 2836, doi: 10.1093/mnras/stz1818
—. 2019b, MNRAS, 488, 2836, doi: 10.1093/mnras/stz1818

McKemmish, L. K., Yurchenko, S. N., \& Tennyson, J. 2016, MNRAS, 463, 771, doi: 10.1093/mnras/stw1969

Melin, S. T., \& Sanders, S. T. 2016, JQSRT, 180, 184 , doi: https://doi.org/10.1016/j.jqsrt.2016.04.009

Merer, A., Huang, G., Cheung, A.-C., \& Taylor, A. 1987, JMS, 125, 465 , doi: https://doi.org/10.1016/0022-2852(87)90110-X

Merritt, S. R., Gibson, N. P., Nugroho, S. K., et al. 2020, A\&A, 636, A117, doi: 10.1051/0004-6361/201937409

Miliordos, E., \& Mavridis, A. 2007, The Journal of Physical Chemistry A, 111, 1953, doi: 10.1021/jp067451b

Molaverdikhani, K., Henning, T., \& Mollière, P. 2019, ApJ, 873, 32, doi: 10.3847/1538-4357/aafda8

Mollière, P., Wardenier, J., van Boekel, R., et al. 2019, Astronomy \& Astrophysics, 627, A67

Mordasini, C., van Boekel, R., Mollière, P., Henning, T., \& Benneke, B. 2016, ApJ, 832, 1.

https://arxiv.org/abs/1609.03019

Ngo, N., Lisak, D., Tran, H., \& Hartmann, J.-M. 2013, JQSRT, 129, 89 ,

doi: https://doi.org/10.1016/j.jqsrt.2013.05.034

Ngo, N. H., Tran, H., Gamache, R. R., \& Hartmann, J. M. 2012, Philosophical Transactions of the Royal Society of London Series A, 370, 2495, doi: 10.1098/rsta.2011.0272

Nugroho, S. K., Gibson, N. P., de Mooij, E. J. W., et al. 2020, MNRAS, 496, 504, doi: 10.1093/mnras/staa1459

Nugroho, S. K., Kawahara, H., Masuda, K., et al. 2017, AJ, 154, 221, doi: 10.3847/1538-3881/aa9433

Öberg, K. I., Murray-Clay, R., \& Bergin, E. A. 2011, ApJL, 743, L16. https://arxiv.org/abs/1110.5567

Odashima, H., Kajita, M., Matsuo, Y., Minowa, T., \& Shimizu, T. 1989, The Journal of Chemical Physics, 90, 4875, doi: 10.1063/1.456581

Partridge, H., \& Schwenke, D. W. 1997, JCP, 106, 4618

Peterson, D. E., Megeath, S. T., Luhman, K. L., et al. 2008, ApJ, 685, 313, doi: 10.1086/590527

Phillips, J. G., Davis, S. P., Lindgren, B., \& Balfour, W. J. 1987a, ApJS, 65, 721, doi: 10.1086/191241

—. 1987b, ApJS, 65, 721, doi: 10.1086/191241

Pickett, H. M. 1980, The Journal of Chemical Physics, 73, 6090, doi: 10.1063/1.440145

Piette, A. A. A., Madhusudhan, N., McKemmish, L. K., et al. 2020, arXiv e-prints, arXiv:2006.04807. https://arxiv.org/abs/2006.04807

Piskorz, D., Buzard, C., Line, M. R., et al. 2018, AJ, 156, 133, doi: 10.3847/1538-3881/aad781

Plez, B. 1998, A\&A, 337, 495

Plez, B. 1999, in Asymptotic Giant Branch Stars, ed. T. Le Bertre, A. Lebre, \& C. Waelkens, Vol. 191, 75 
Plez, B., Brett, J. M., \& Nordlund, A. 1992, A\&A, 256, 551

Polyansky, O. L., Kyuberis, A. A., Zobov, N. F., et al. 2018, MNRAS, 480, 2597, doi: 10.1093/mnras/sty1877

Polyansky, O. L., Zobov, N. F., Mizus, I. I., et al. 2018, JQSRT, 210, 127

Ram, R., Bernath, P., Davis, S., \& Merer, A. 2002, JMS, 211, 279 , doi: https://doi.org/10.1006/jmsp.2001.8510

Ram, R., Bernath, P., \& Wallace, L. 1996, ApJ Supplement Series, 107, 443

Ram, R. S., \& Bernath, P. F. 1996, ApOpt, 35, 2879, doi: 10.1364/AO.35.002879

—. 2005, JMS, 229, 57, doi: 10.1016/j.jms.2004.08.014

Ram, R. S., Bernath, P. F., Dulick, M., \& Wallace, L. 1999, ApJS, 122, 331, doi: 10.1086/313212

Ram, R. S., Jarman, C. N., \& Bernath, P. F. 1993, JMS, 161, 445, doi: 10.1006/jmsp.1993.1250

Ram, R. S., Tereszchuk, K., Gordon, I. E., Walker, K. A., \& Bernath, P. F. 2011, JMS, 266, 86, doi: 10.1016/j.jms.2011.03.009

Reid, I. N., Burgasser, A. J., Cruz, K. L., Kirkpatrick, J. D., \& Gizis, J. E. 2001, AJ, 121, 1710, doi: 10.1086/319418

Reid, I. N., \& Hawley, S. L. 2005, New light on dark stars : red dwarfs, low-mass stars, brown dwarfs (Praxis Publishing Ltd), doi: 10.1007/3-540-27610-6

Renaud, C. L., Cleghorn, K., Hartmann, L., Vispoel, B., \& Gamache, R. R. 2018, Icarus, 306, 275, doi: 10.1016/j.icarus.2017.10.016

Robert, D., \& Bonamy, J. 1979, Journal de Physique, 40, 923

Rothman, L., Gordon, I., Barber, R., et al. 2010, JQSRT, 111, 2139

Rothman, L. S., Jacquemart, D., Barbe, A., et al. 2005, Journal of quantitative spectroscopy and radiative transfer, 96, 139

Ruiz, M. T., Leggett, S. K., \& Allard, F. 1997, ApJL, 491, L107, doi: 10.1086/311070

Régalia-Jarlot, L., Thomas, X., Von der Heyden, P., \& Barbe, A. 2005, Journal of Quantitative Spectroscopy and Radiative Transfer, 91, 121 , doi: https://doi.org/10.1016/j.jqsrt.2004.05.042

Schiavon, R. P., Barbuy, B., \& Singh, P. D. 1997, ApJ, 484, 499, doi: 10.1086/304332

Schreier, F. 2018a, MNRAS, 479, 3068, doi: $10.1093 / \mathrm{mnras} /$ sty 1680

—. 2018b, JQSRT, 213, 13, doi: 10.1016/j.jqsrt.2018.03.019

Schwenke, D. W. 1998, Faraday Discussions, 109, 321

Schwenke, D. W. 1998, Faraday Discussions, 109, 321, doi: 10.1039/a800070k

Sharp, C. M., \& Burrows, A. 2007, ApJS, 168, 140. https://arxiv.org/abs/0607211
Sharpless, S. 1956, ApJ, 124, 342, doi: 10.1086/146229

Shayesteh, A., Alavi, S. F., Rahman, M., \& Gharib-Nezhad, E. 2017, Chemical Physics Letters, 667, 345, doi: 10.1016/j.cplett.2016.11.020

Shayesteh, A., \& Bernath, P. F. 2011, JChPh, 135, 094308, doi: 10.1063/1.3631341

Shayesteh, A., Ram, R. S., \& Bernath, P. F. 2013, JMS, 288, 46, doi: 10.1016/j.jms.2013.04.009

Shayesteh, A., Walker, K. A., Gordon, I., Appadoo, D. R. T., \& Bernath, P. F. 2004, Journal of Molecular Structure, 695-696, 23, doi: 10.1016/j.molstruc.2003.11.001

Sonnabend, G., Wirtz, D., Schieder, R., \& Bernath, P. F. 2006, Solar Physics, 233, 205, doi: 10.1007/s11207-006-2488-9

Sousa-Silva, C., Hesketh, N., Yurchenko, S. N., Hill, C., \& Tennyson, J. 2014, JQSRT, 142, 66, doi: 10.1016/j.jqsrt.2014.03.012

Steimle, T. C., Shirley, J. E., Simard, B., Vasseur, M., \& Hackett, P. 1991, JChPh, 95, 7179, doi: 10.1063/1.461394

Steimle, T. C., \& Virgo, W. 2003, Chemical Physics Letters, 381, 30, doi: 10.1016/j.cplett.2003.09.102

Sullivan, P. W., Winn, J. N., Berta-Thompson, Z. K., et al. 2015, ApJ, 809, 77. https://arxiv.org/abs/1506.03845

Szajna, W., \& Zachwieja, M. 2009, European Physical Journal D, 55, 549, doi: 10.1140/epjd/e2009-00253-y

—. 2010, JMS, 260, 130, doi: 10.1016/j.jms.2010.02.004

Tan, Y., Kochanov, R. V., Rothman, L. S., \& Gordon, I. E. 2019, Journal of Geophysical Research: Atmospheres, 124, 11580, doi: 10.1029/2019JD030929

Tennyson, J., Yurchenko, S. N., Al-Refaie, A. F., et al. 2020, JQSRT, 255, 107228, doi: 10.1016/j.jqsrt.2020.107228

Tinney, C. G., \& Reid, I. N. 1998, MNRAS, 301, 1031, doi: 10.1046/j.1365-8711.1998.02079.x

Toth, R. A., Hunt, R. H., \& Plyler, E. K. 1970, JChPh, 53, 4303, doi: 10.1063/1.1673937

van den Bekerom, D. C. M., \& Pannier, E. 2021, JQSRT, 261, 107476, doi: 10.1016/j.jqsrt.2020.107476

Veillard, A. 2012, Quantum chemistry: the challenge of transition metals and coordination chemistry, Vol. 176 (Springer Science \& Business Media)

Vidler, M., \& Tennyson, J. 2000, JChPh, 113, 9766, doi: $10.1063 / 1.1321769$

Villanueva, G., Smith, M., Protopapa, S., Faggi, S., \& Mandell, A. 2018, JQSRT, 217, 86 , doi: https://doi.org/10.1016/j.jqsrt.2018.05.023

Vispoel, B., Cavalcanti, J. H., Paige, E. T., \& Gamache, R. R. 2020, JQSRT, 253, 107030, doi: https://doi.org/10.1016/j.jqsrt.2020.107030 
Visscher, C., Lodders, K., \& Fegley, Bruce, J. 2010, ApJ, 716, 1060, doi: 10.1088/0004-637X/716/2/1060

Wagner, G., Birk, M., Gamache, R. R., \& Hartmann, J. M. 2005, JQSRT, 92, 211, doi: 10.1016/j.jqsrt.2004.07.023

Watson, W. W., \& Hull, G. F. 1936, Physical Review, 49, 592, doi: 10.1103/PhysRev.49.592

Webber, J. C. 1971, SoPh, 16, 340, doi: $10.1007 / \mathrm{BF} 00162476$

Weck, P. F., Stancil, P. C., \& Kirby, K. 2003, JChPh, 118, 9997, doi: 10.1063/1.1573181

Wende, S., Reiners, A., Seifahrt, A., \& Bernath, P. F. 2010, A\&A, 523, A58, doi: 10.1051/0004-6361/201015220

Yadin, B., Veness, T., Conti, P., et al. 2012, MNRAS, 425, 34, doi: 10.1111/j.1365-2966.2012.21367.x
Yang, B., Zhang, P., Qu, C., et al. 2018, The Journal of Physical Chemistry A, 122, 1511

Yurchenko, S. N., Al-Refaie, A. F., \& Tennyson, J. 2018, A\&A, 614, A131, doi: 10.1051/0004-6361/201732531

Yurchenko, S. N., Williams, H., Leyland, P. C., Lodi, L., \& Tennyson, J. 2018, MNRAS, 479, 1401, doi: 10.1093/mnras/sty1524

Zaghloul, M. R. 2015, arXiv preprint arXiv:1505.05596

Zhang, X. 2020, Research in Astronomy and Astrophysics, 20, 099, doi: 10.1088/1674-4527/20/7/99 